\title{
Review Article \\ Cytochrome c: A Multifunctional Protein Combining Conformational Rigidity with Flexibility
}

\author{
Reinhard Schweitzer-Stenner \\ Department of Chemistry, Drexel University, 3141 Chestnut Street, Philadelphia, PA 19104, USA \\ Correspondence should be addressed to Reinhard Schweitzer-Stenner; rschweitzer-stenner@drexel.edu
}

Received 26 February 2014; Accepted 3 June 2014; Published 22 July 2014

Academic Editor: Hamid Mobasheri

Copyright () 2014 Reinhard Schweitzer-Stenner. This is an open access article distributed under the Creative Commons Attribution License, which permits unrestricted use, distribution, and reproduction in any medium, provided the original work is properly cited.

\begin{abstract}
Cytochrome has served as a model system for studying redox reactions, protein folding, and more recently peroxidase activity induced by partial unfolding on membranes. This review illuminates some important aspects of the research on this biomolecule. The first part summarizes the results of structural analyses of its active site. Owing to heme-protein interactions the heme group is subject to both in-plane and out-of-plane deformations. The unfolding of the protein as discussed in detail in the second part of this review can be induced by changes of $\mathrm{pH}$ and temperature and most prominently by the addition of denaturing agents. Both the kinetic and thermodynamic folding and unfolding involve intermediate states with regard to all unfolding conditions. If allowed to sit at alkaline $\mathrm{pH}$ (11.5) for a week, the protein does not return to its folding state when the solvent is switched back to neutral $\mathrm{pH}$. It rather adopts a misfolded state that is prone to aggregation via domain swapping. On the surface of cardiolipin containing liposomes, the protein can adopt a variety of partially unfolded states. Apparently, ferricytochrome c can perform biological functions even if it is only partially folded.
\end{abstract}

\section{Introduction}

Among the multitude of biomolecules, the family of heme proteins has played a peculiar and prominent role in biophysical research over many decades [1]. All members of this family contain at least one, sometimes multiple, heme group as active sites. Heme groups are iron-protoporphyrin derivatives. Figure 1 exhibits heme $c$, the prosthetic group of the electron carrier cytochrome $c$. The simplest heme proteins are monomers with a variety of secondary and tertiary structures, the former being dominated by helical segments in most cases (Figure 2). However, for some of these proteins the monomers assemble to form a quaternary structure. The most prominent example is hemoglobin, which contains four globular heme proteins as subunits. This quaternary structure is pivotal for the protein's biological function in that it enables a cooperative communication between the four binding sites, which allows for an effective uptake and release of oxygen in the lungs and the tissue, respectively.

All heme proteins contain a heme group which functions as their active site for performing a diverse set of tasks, such as ligand binding and transfer (myoglobin and hemoglobin)
[2], electron transfer (cytochrome reductase and oxidase, cytochrome c) [3, 4], facilitation of enzymatic reactions catalyzing peroxidase (horseradish peroxidase) [5], monooxygenase (cytochrome P450) [6], cyclase (guanylate cyclase) [7], and catalase reactions [8], and signaling process involving the binding and release of nitric oxide (nitrophorin) [9]. The individual functions of these heme groups are regulated by the axial ligands of their heme iron, multiple noncovalent interactions between the heme (porphyrin) macrocycle and the protein environment, and in the case of members of the cytochrome $\mathrm{c}$ family even covalent bonds between peripheral substituents of the heme and protein side chains (Figure 1) [4]. Thus, the protein creates a rather asymmetric and chiral environment which affects the symmetry of the macrocycle and also the electronic structure of the central metal $[10,11]$. All heme groups exhibit a rather strong absorption in the visible region reminiscent of color centers in crystals.

This review provides an overview on work performed in our laboratories over the last twenty years (University of Bremen, University of Puerto Rico, and Drexel University) which was aimed at exploring cytochrome $\mathrm{c}$ function, dynamics, and folding. In spite of nearly 60 years of research, 


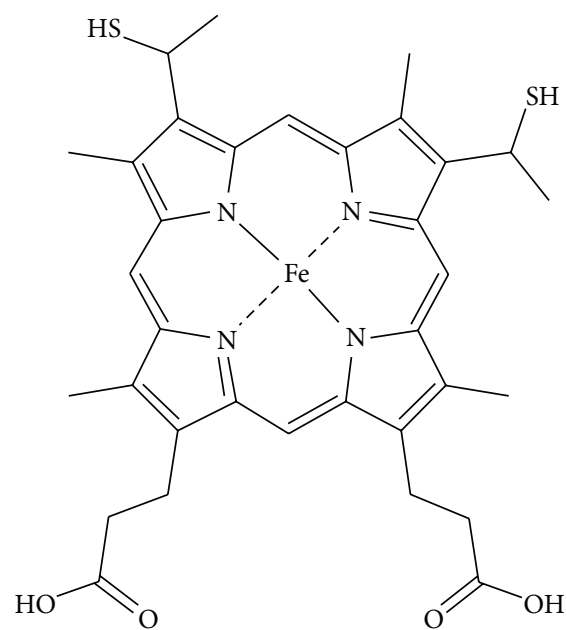

FIGURE 1: Structure of heme c, the prosthetic group in cytochrome c.

cytochrome $\mathrm{c}$ is still of great interest to biophysicists and biochemists alike owing to its multifunctionality: it serves as an electron carrier between major membrane proteins in the inner membrane of the mitochondria [4], it functions as a scavenger for $\mathrm{H}_{2} \mathrm{O}_{2}$ as peroxidase [12-15], and it functions as an initiator of a biochemical cascade which finally causes mitochondrial apoptosis [15-17]. The protein has also been utilized as a model system for protein folding studies [18]. The research in our laboratories has mostly been focused on elucidating heme-protein interactions and asymmetric deformations of the heme macrocycle [11, 19], but lately we used spectroscopic means to probe partially unfolded states of the protein [20]. The second chapter of this review describes how heme-protein interactions affecting the prosthetic heme group of cytochrome can be probed by resonance Raman, visible circular dichroism, and low temperature absorption spectroscopy. The third chapter reviews the studies of folding and unfolding triggered by $\mathrm{pH}$ and temperature change. This includes the recent discovery of a partially unfolded state which remains stable at conditions that normally favor a native, fully folded state (neutral $\mathrm{pH}$, room temperature). In the short fourth section we briefly review recent results of our studies on cytochrome c-liposome interactions. Cardiolipincontaining liposomes serve as model systems for the inner membrane mitochondria in these studies [21].

\section{Heme-Protein Interactions in Cytochrome c}

2.1. Secondary and Tertiary Structure. Figure 3 shows the crystal structure of horse heart ferri-cytochrome c. The coloring of the different structural segments follows a code introduced by Englander and coworkers [18, 23]. Its significance for the understanding of the protein's folding is explained below. Cytochrome $\mathrm{c}$ is a globular protein with an $\alpha$-helical fraction of ca. $40 \%[25,26]$. That value is lower than what one observes for the classical heme proteins myoglobin and hemoglobin [2]. The two main helical sections are the socalled $\mathrm{N}$ - and C-terminal helices. Noncovalent interactions

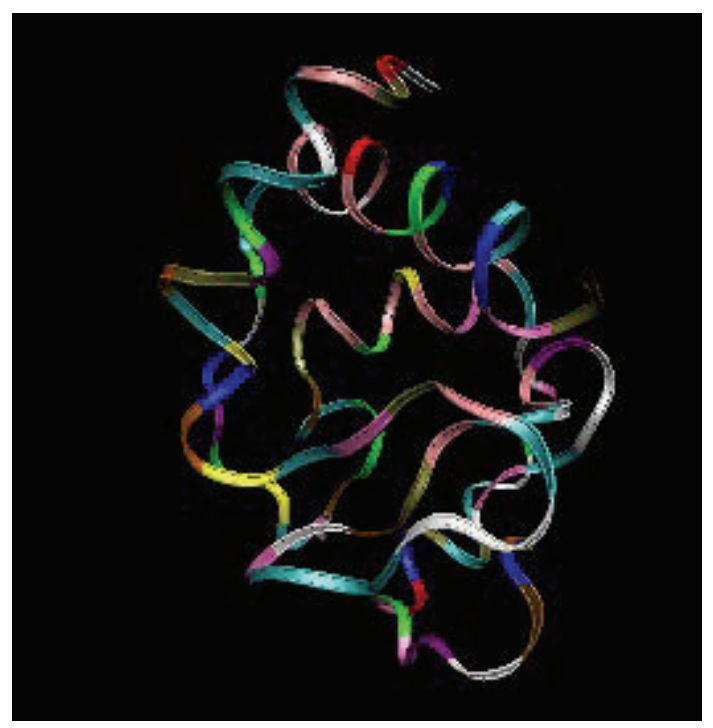

FIGURE 2: Visualization of the secondary and tertiary structures of ferro-horse heart cytochrome c obtained from pdb 2FRC. The figure has been produced with VMD software [22].

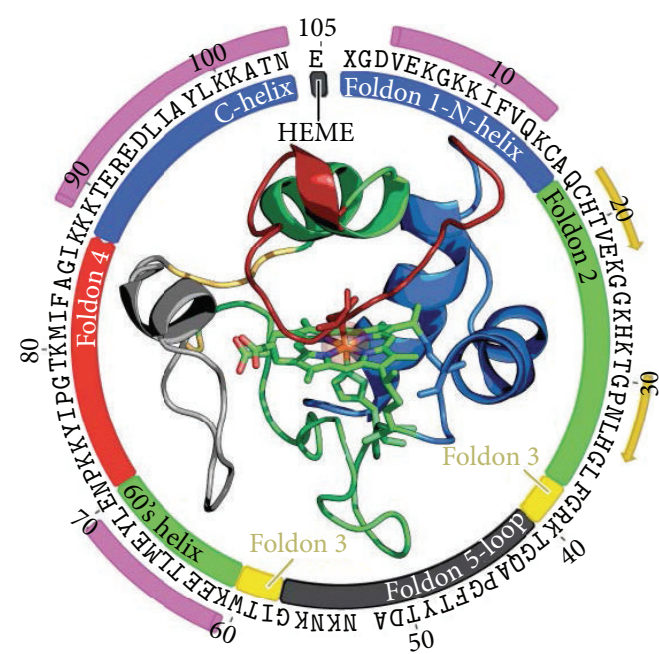

FIGURE 3: Structure of oxidized horse heart cytochrome c presented with a color scheme that illustrates the different foldons identified by Krishna and coworkers [23]. These foldons and the respective color code are explained in detail in the text. The outside circle provides the primary amino acid sequence of the different protein segments. The figure has been taken from Soffer [24]. The original figure exhibiting the different foldons can be found in [23].

between these two helices are pivotal for the stabilization of the native state [18, 27-29]. A third helix (the so-called 60's helix) is much shorter than the $\mathrm{C}$ - and $\mathrm{N}$-helices. The rest of the protein comprises rather long loop segments. Two of them are of particular importance. Firstly, the loop starting from the N-terminal helix contains H18, the imidazole side chain of which provides the proximal ligand of the heme iron. The CxyC motif with the two cysteins covalently linked to the heme via thioether bridges is located between H18 and the $\mathrm{N}$-terminal helix. The notation xy indicates different amino 
acid residues in different cytochrome $\mathrm{c}$ derivatives. In horse heart cytochrome c, the segment reads as CAQC. Secondly, the red loop region in Figure 3, the $\Omega$-loop, contains the M80, the sulfur atom of which provides the second axial ligand for the heme iron.

\subsection{Asymmetric Deformations of the Functional Heme Group.} In spite of a rather asymmetric heme environment and strong heme-protein interactions via the two axial ligands [10, 30, 31], the thioether bridges [32], the internal electric field at the heme [33-38], and to a lesser extent substituent-hydrogen bonding $[25,26,39-41]$ and multiple van der Waals contacts [42], spectroscopists have for a long period of time considered the heme as exhibiting an ideal $\mathrm{D}_{4 \mathrm{~h}}$-symmetry. This was (and in part still is) particularly true for the interpretation of optical absorption and resonance Raman data [43-49]. This view ignores very early EPR data for many paramagnetic heme proteins including ferricytochrome $\mathrm{c}$ that revealed a rhombic deformation of the heme iron's ligand field, which reflects deformations of the heme group assignable to the irreducible representation $B_{1 g}$ of the $\mathrm{D}_{4 \mathrm{~h}}$-point group [10,30, 50]. Such a deformation lowers the overall symmetry of the heme to $\mathrm{C}_{2 \mathrm{v}}$. Chemical shift distributions in, for example, ${ }^{1} \mathrm{H}$ NMR spectra, of methyl groups revealed the nonequivalence of the methyl substituents of the heme and thus a heme symmetry much lower than $\mathrm{D}_{4 \mathrm{~h}}[30,51,52]$. Senn et al. reported NMR-data that revealed a rather asymmetric spin density distribution of the heme macrocycle in a cytochrome c derivative [53]. However, none of these studies involved a systematic investigation of heme deformations. This task was first carried out by resonance Raman dispersion spectroscopy (RRDS), the results of which are described in detail in the following.

Already in the seventies, Collins et al. [54] reported that the depolarization ratios of structure sensitive bands in the resonance Raman spectrum of horse heart ferrocytochrome c show a rather strong dispersion in the Q-band region of the optical spectrum exhibited in Figure 4. Thus, they clearly deviate from their $\mathrm{D}_{4 \mathrm{~h}}$-expectation values, which do not depend on the choice of the excitation wavelength [43]. Shelnutt as well as Zgierski and Pawlikowski explained these dispersions in terms of electronic and vibronic perturbations, which will be explained below $[55,56]$. A systematic investigation of Raman depolarization ratio dispersions and resonance excitation profiles of various heme proteins was subsequently in our research group at the University of Bremen [57-60]. For ferrocytochrome c, we reported dispersion and excitation data over a rather broad range of excitation wavelengths that encompasses the entire Q-band region [60]. As an example, Figure 5 shows the depolarization dispersion and resonance excitation profile of the oxidation marker $v_{4}$. It is the dominant band in spectra taken with $B$-(Soret) band excitation, while it is comparatively weak in spectra taken with Q-band excitation (cf. the optical spectrum in Figure 4) [61]. The eigenvector of the corresponding normal mode is shown in Figure 6. It involves $\mathrm{C}_{\alpha}-\mathrm{C}_{\beta}$ and $\mathrm{C}_{\alpha}-\mathrm{N}$ stretching modes, which make it sensitive to axial ligands induced

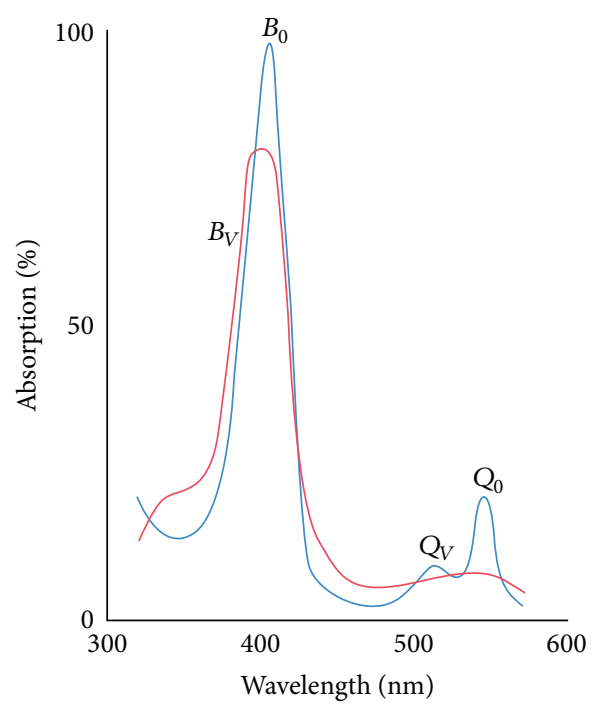

FIGURE 4: Visible absorption spectra of cytochrome c: ferrocytochrome c (blue) and ferricytochrome c (red). Taken from [64] and modified.

asymmetric deformations of the heme [11]. In an ideal $\mathrm{D}_{4 \mathrm{~h}}{ }^{-}$ symmetry, the depolarization ratio of this band is 0.125 , indicative of the $A_{1 g}$-symmetry of its eigenvector (Figure 6) [43]. Raman excitation of this band is generally assumed to be exclusively governed by Franck-Condon type vibronic coupling describable by Albrecht's A-term contribution to the Raman tensor [43, 45, 48, 49]. If this was the case, the band would be practically undetectable in the entire preresonance and resonance regions of the Q-band, contrary to what the excitation profile in Figure 5 suggests. In reality, the $v_{4}{ }^{-}$ band gains considerable Raman activity from Herzberg-Teller coupling $[19,60,62,63]$.

The underlying theory utilized to analyze the data in Figure 5 is rather complex and has been described in several papers and comprehensive review articles [11, 19]. Since this paper focuses on structural and related functional properties of cytochrome c, we consider only the structural information that emerged from the analysis of the Raman data. If one neglects multimode mixing and stays in the regime of weak coupling limit, the Raman cross section will be proportional to the squares of the following vibronic coupling matrix elements:

$$
c_{e s}^{\Gamma_{r}}=\left\langle e\left|\frac{\partial \widehat{H}_{e l}}{\partial Q^{\Gamma_{r}}}\right| s\right\rangle Q_{01}^{\Gamma_{r}},
$$

where $\widehat{H}_{e l}$ is the electronic Hamiltonian which also contains the Coulomb interactions between the nuclei. $Q_{01}^{\Gamma_{r}}$ is transition matrix element of the vibrational $0 \rightarrow 1$ transition for the normal coordinate $Q^{\Gamma_{r}}$ of the $r$ th Raman active vibration exhibiting the symmetry $\Gamma_{r}$ in an ideal $\mathrm{D}_{4 \mathrm{~h}}$-symmetry. Equation (1) describes the vibronic coupling between excited electronic states $|s\rangle$ and $|e\rangle$, which for porphyrins can be equated with $\left|B_{x}\right\rangle,\left|B_{y}\right\rangle,\left|Q_{x}\right\rangle$, and $\left|Q_{y}\right\rangle$. In $\mathrm{D}_{4 \mathrm{~h}}$, the $B$ - and $Q$-states are twofold degenerates, that is, $E_{B x}=E_{B y}$ and $E_{\mathrm{Q} x}=E_{\mathrm{Q} y}$. Since $Q$ - and $B$-states exhibit $E_{u}$-symmetry, 


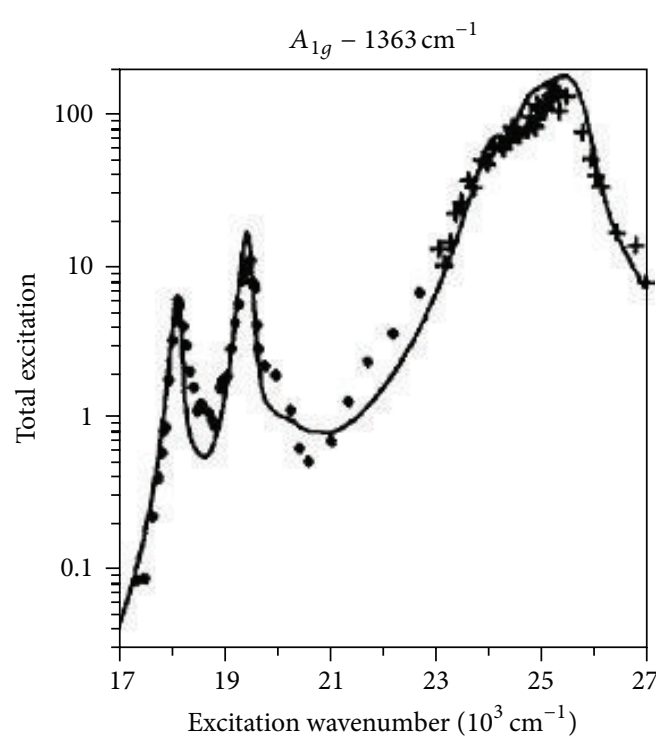

(a)

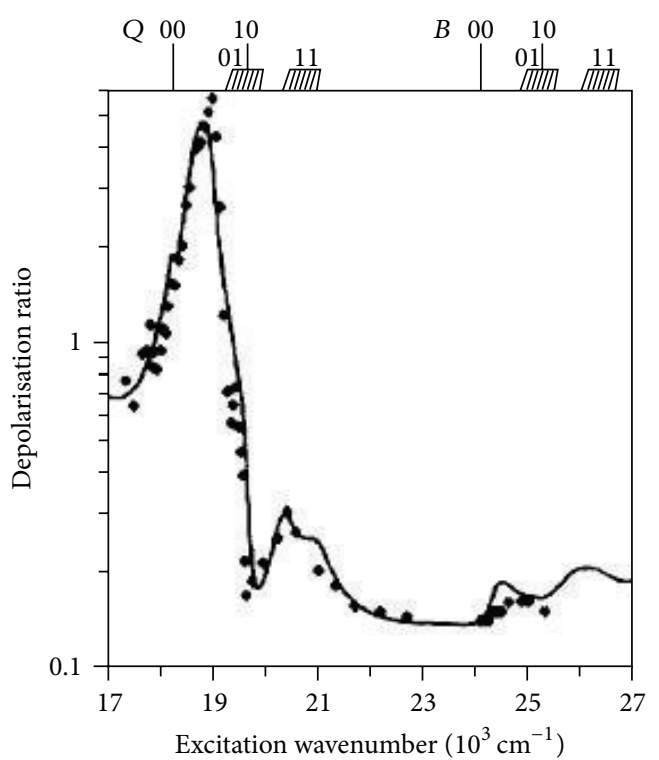

(b)

FIGURE 5: Depolarization ratio dispersion (left) and resonance excitation profile of the $v_{4}$-band of horse heart ferrocytochrome $\mathrm{c}$ in solution at $\mathrm{pH}$ 7. The solid line results from a multimode fitting procedure described by Schweitzer-Stenner et al. [60], from where the figure was taken with permission. The data in the Soret band region of the resonance excitation profile have been measured earlier by Champion and Albrecht [49].

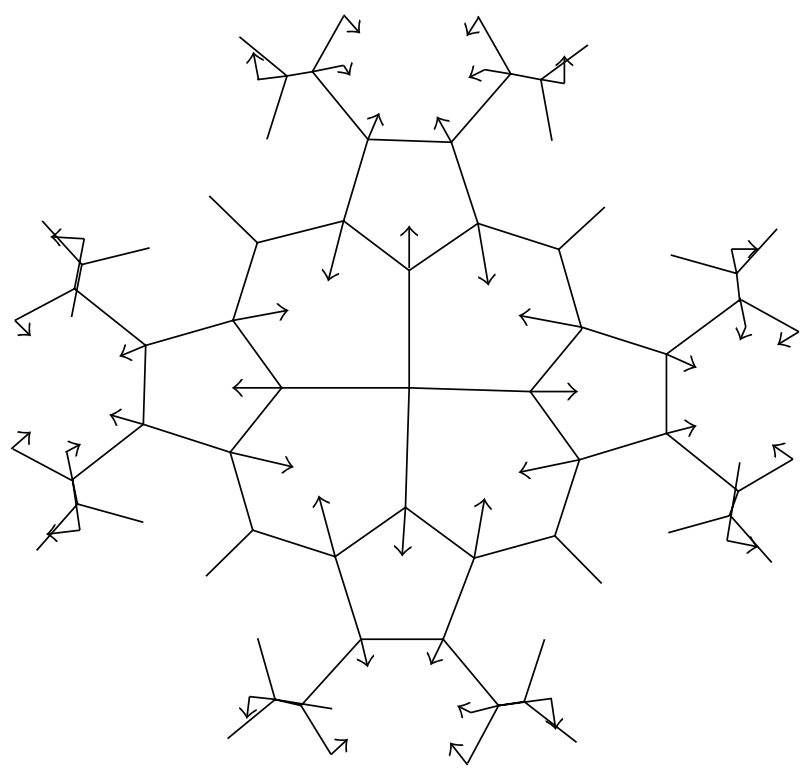

FIGURE 6: Normal mode pattern of the $v_{4}$-mode of $\mathrm{Ni}$ (II)octaethylporphyrin, a canonical model system for the vibrational analysis of metal porphyrins. The analysis was carried out by Dr. Esko Unger in the author's former research group at the University of Bremen and has not been published. The theoretical approach used for this analysis has been described by Unger et al. [62]

only vibrations of $A_{1 g}, B_{1 g}, B_{2 g}$, and $A_{2 g}$ can contribute to vibronic coupling. Thus, only modes exhibiting one of these symmetries can be resonance Raman active. They can be distinguished by their excitation wavelength independent depolarization ratios of $0.125,0.75,0.75$, and $\infty$ [43, 44, 65]. $B_{1 g^{-}}, B_{2 g^{-}}$, and $A_{2 g^{-}}$-modes are predominantly though not exclusively Herzberg-Teller active; this type of coupling mixes components of different electronic states $\left(Q_{x}, Q_{y}\right.$ with $B_{x}$, $B_{y}$, in our case). Generally, modes of different symmetry can be selectively enhanced with different excitation wavelengths. This is illustrated by the resonance Raman spectra of yeast ferrocytochrome $\mathrm{c}$ in Figure 7. The (polarized) spectrum taken with $442 \mathrm{~nm}$ excitation is dominated by Franck-Condon $\left(A_{1 g}\right)$ and to a lesser extent Jahn-Teller type $\left(B_{1 g}\right)$ coupling to the $B$-band transitions. Raman bands in the $521 \mathrm{~nm}$ spectrum $\left(Q_{v}\right.$ excitation) are mostly depolarized and are assignable to both $B_{1 g}$ and $B_{2 g}$ modes, which both gain their Raman excitation mostly from Herzberg-Teller coupling. The $531 \mathrm{~nm}$ excitation lies between $Q_{0}$ and $Q_{v}$ resonances and is dominated by inverse polarized bands assignable to $A_{2 g}$-modes. In this spectral region, Herzberg-Teller coupling in this region exhibits constructive interference for $A_{2 g^{-}}$ modes and destructive interference for $A_{1 g^{-}}, B_{1 g^{-}}$, and $B_{2 g^{-}}$ modes. Excitation with $568 \mathrm{~nm}$ coincides with the $Q_{o}$-band region. Here, constructive interferences between Jahn-Teller and Herzberg-Teller coupling make bands assignable to $B_{1 g^{-}}$ modes dominate the spectrum [62].

As indicated above, the real symmetry of a heme group is not $\mathrm{D}_{4 \mathrm{~h}}$. This implies that a perturbation potential $V$ has to be added to $\widehat{H}_{e l}$ that is produced by the peripheral substituents and the protein environment $[19,66]$ :

$$
c_{e s}^{\Gamma_{r^{\prime}}}=\left\langle e\left|\frac{\partial \widehat{H}_{e l}}{\partial Q^{\Gamma_{r}}}+\sum_{j} \frac{\partial V^{\Gamma_{j}}}{\partial Q^{\Gamma_{r}}}\right| s\right\rangle Q_{01}^{\Gamma_{r}},
$$


where the electronic perturbation $V^{\Gamma_{j}}$ is classified in terms of irreducible representations $\Gamma_{j}$ of the $D_{4 h}$ point group. This approach, which was first introduced by Shelnutt [55], assumes that the perturbation of the heme group is not large enough to eliminate all resemblance with the $\mathrm{D}_{4 \mathrm{~h}}$ point group. While $V^{\Gamma_{j}}$ does not depend on the chosen normal vibration, the derivative of this potential in (2) is different for each Raman active vibration. Following Zgierski and Pawlikowski, we call this contribution vibronic perturbation [56].

Based on the assumption that $V \ll H_{e l}$, we expressed the perturbation potential in terms of so-called normal coordinate deformations $\delta Q^{\Gamma_{j}}[11]$ :

$$
V^{\Gamma_{j}}=\frac{\partial \widehat{H}_{e l}}{\partial Q^{\Gamma_{j}}} \delta Q^{\Gamma_{j}} .
$$

As later shown by Jentzen et al., each possible distortion from $\mathrm{D}_{4 \mathrm{~h}}$ is describable as a superposition of deformations along normal coordinates of the porphyrin macrocycle [67]. For energetic reasons, coordinates of modes exhibiting the lowest frequency for a given symmetry $\Gamma_{j}$ contribute predominantly to the overall heme deformation. The patterns of such deformations, which can be subdivided into in-plane and out-of-plane deformations, are shown in Figure 8. Inplane deformations exhibit $A_{1 g^{-}}, B_{1 g^{-}}, B_{2 g^{-}}, A_{2 g^{-}}$, and $E_{u^{-}}$ symmetry. In what follows we will focus on $B_{1 g^{-}}$and $B_{2 g^{-}}$ type deformations. The former involves the pyrrole nitrogens and produces a rhombic deformation of the iron's crystal field. $B_{2 g}$-deformation affects the meso-carbons; the formed deformation can be described as triclinic. Out-of-plane deformations transform like $A_{1 u}, B_{1 u}, B_{2 u}, A_{2 u}$, and $E_{g}$, which Jentzen et al. termed propellering, ruffling, saddling, doming, and waving. In what follows we will focus on ruffling and saddling, which both affect the relative orientation of the pyrroles rather than the metal position $[32,67]$.

The relationship between $V$ and $\delta Q$ is that between cause and effect. A perturbing potential exerted by substituents and the protein environment forces the heme group into a new equilibrium structure that can be constructed from the pure $\mathrm{D}_{4 \mathrm{~h}}$-conformation by subjecting the latter to a linear combination of $\delta Q s$.

Apparently, the occurrence of in-plane (ip) and out-ofplane (oop) deformations mixes contributions of different symmetries into the vibronic coupling matrix elements. While the first term in (1) transforms like the irreducible representation of $Q^{\Gamma_{r}}$ in $D_{4 h}$, the second one adds product representations $\Gamma_{r} \otimes \Gamma_{j}$. This mixing of symmetries changes the depolarization ratio of the Raman mode. Combined with some interference between Franck-Condon, Jahn-Teller, and Herzberg-Teller coupling, this causes a dispersion of the depolarization ratio, as shown in Figure 5 for the $A_{1}$-mode $v_{4}[60]$.

The depolarization dispersion of $v_{4}$ can be used for a qualitative assessment of the deformations of the heme symmetry in ferrocytochrome c. In $\mathrm{D}_{4 \mathrm{~h}}$, the depolarization ratio is 0.125 . The experimental data suggest that it is larger at all excitation wavelengths. Any in-plane deformation of

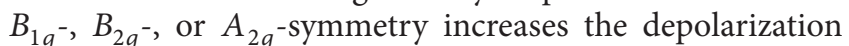
ratio. Only $A_{2 g}$-deformations can increase it above 0.75 , what

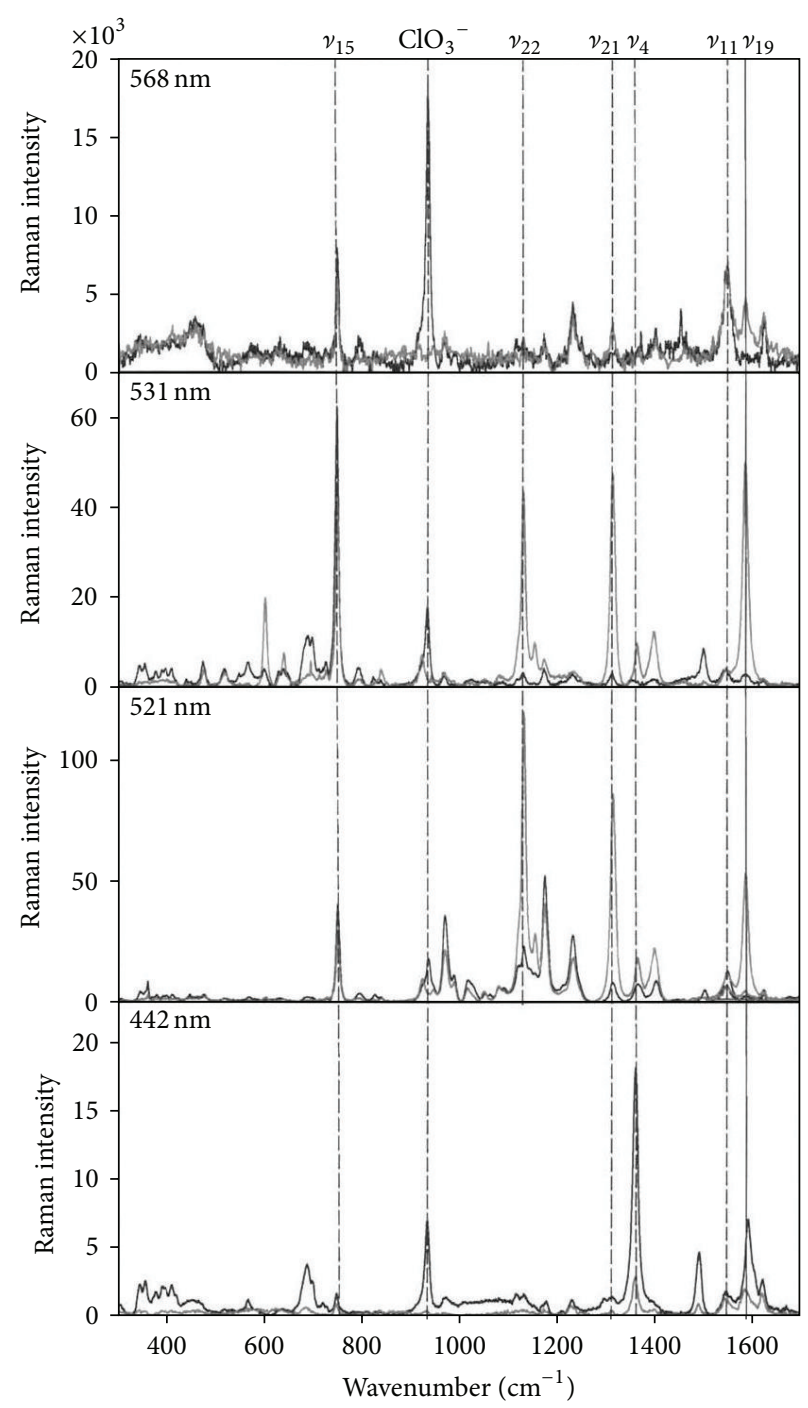

FIGURE 7: Polarized resonance Raman spectra of horse heart ferrocytochrome $\mathrm{c}$ in aqueous solution taken at the indicated excitation wavelengths. Spectra recorded with $x$-polarization (parallel polarized to the exciting laser beam) are displayed in black and those with $y$-polarization (perpendicular polarized to the exciting laser beam) are displayed in gray. $B$-band excitation was achieved with $442 \mathrm{~nm}$ excitation. Excitation wavelengths 521, 531, and $568 \mathrm{~nm}$ correspond to $Q_{v}$-band, the region between $Q_{0}$ and $Q_{v}$ band, and $Q_{0}$ band excitation, respectively. Taken from [61] with permission.

indeed happens for $v_{4}$ (cf. Figure 5) in the region between the $Q_{0}$ and the $Q_{v}$ resonances. However, the contribution of antisymmetric vibronic coupling is negligible outside of the Q-band region $[19,43]$. Thus the dispersion between $Q$ - and $B$-band region must result from $B_{1 g^{-}}$and $B_{2 g^{-}}$-type deformations. While the depolarization of the $v_{4}$-mode does not allow for distinguishing between $B_{1 g}$ and $B_{2 g}$, this task can be accomplished by measuring the depolarization ratios of $B_{1 g^{-}}$and $B_{2 g}$-modes $\left(v_{10}, v_{11}\right)$ [41, 59]. Respective data clearly indicate that both types of deformations affect the heme group of ferro- and ferricytochrome c [41].

Thus far we have interpreted deviations of the depolarization ratio from its $\mathrm{D}_{4 \mathrm{~h}}$ value solely in terms of in-plane 

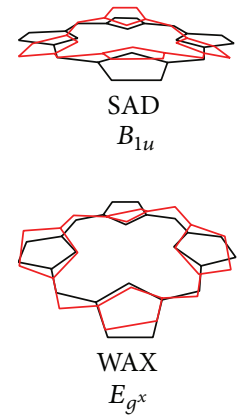

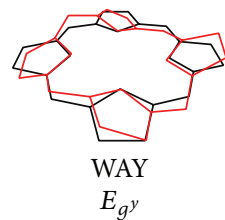

(a)
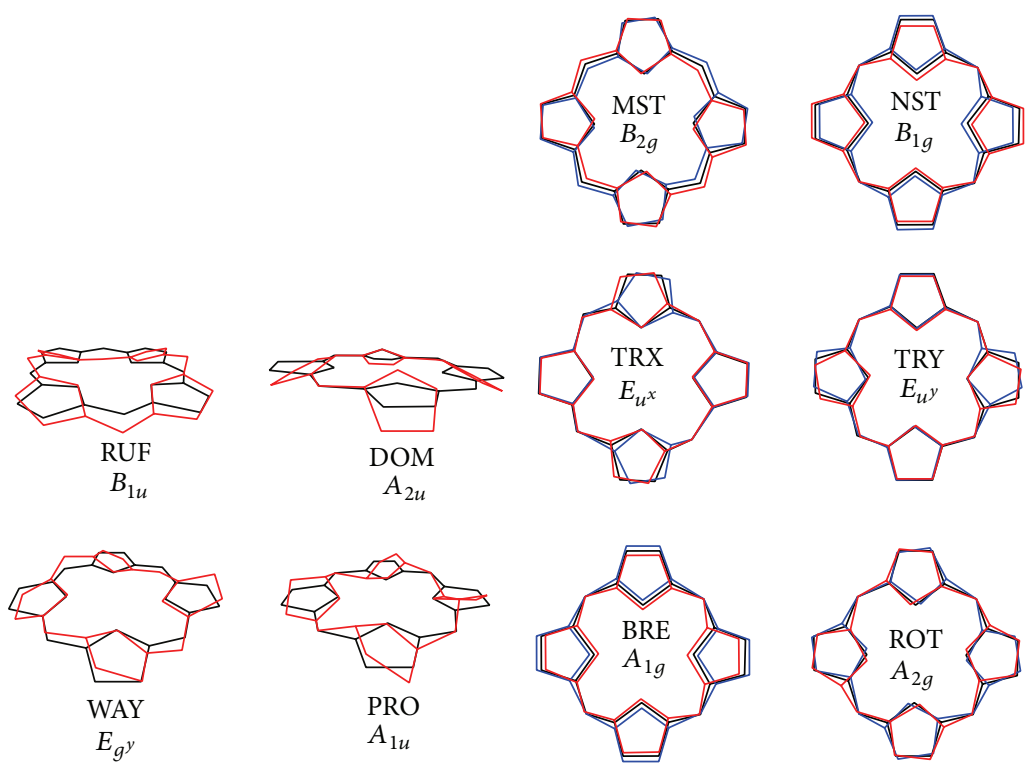

(b)

FIGURE 8: Representations of (a) in-plane and (b) out-of-plane deformations of the porphyrin macrocycle, classified in terms of the irreducible representations of the $\mathrm{D}_{4 \mathrm{~h}}$ point group. Each of these deformations reflects the normal coordinate pattern of the lowest frequency mode of the respective symmetry. Taken form [67] and modified.

deformations. However, for cytochrome $\mathrm{c}$ this would lead to the conclusion that antisymmetric $A_{2 g}$-type deformations are dominant. This is at odds with two observations. Firstly, the lowest frequency of $A_{2 g}$-modes of the porphyrin macrocycle lies well above respective values for $B_{1 g}$ and $B_{2 g}$ [65], which makes it much more difficult to deform the heme along the former than along the latter. Secondly, a decomposition of the deviation of the cytochrome heme group in the crystal structure of the protein from its ideal $\mathrm{D}_{4 \mathrm{~h}}$-symmetry into normal coordinate deformations (called normal mode structural decomposition, NSD) revealed very weak $A_{2 q}$-deformations [67]. The reason for the observed large depolarization ratio of the $v_{4}$-band lies in the existence of rather significant oop-deformations of the heme group of cytochrome c [32, 67]. These deformations have escaped the attention of crystallographers for decades, but they were finally discovered and analyzed by concerted efforts of Jentzen, Shelnutt, and coworkers [32, 67-70]. These researchers developed NSD as a tool of structural exploration of heme chromophores of a large number of heme proteins [67]. For ferro- and ferricytochrome $c$, they inferred a larger ruffling from the crystallographic data. This is followed by saddling, which exhibits $B_{2 u}$-symmetry. Figure 9 visualizes the result of the NSD analysis of the heme group in horse heart cytochrome c. Together, they lower the symmetry from $\mathrm{D}_{4 \mathrm{~h}}$ to $S_{4}$. If both of these deformations are present, they can affect vibronic coupling via the second order term $[63,71]$ :

$$
V^{\Gamma^{*}}=\frac{\partial^{2} \widehat{H}_{e l}}{\partial Q^{B_{1 u}} \partial Q^{B_{2 u}}} \delta Q^{B_{1 u}} \delta Q^{B_{2 u}},
$$

where $\delta Q^{B_{1 u}}$ and $\delta Q^{B_{2 u}}$ denote ruffling and saddling normal deformations. The electronic part of (4) transforms like $B_{1 u} \otimes$
$B_{2 u}=A_{2 g}$, which explains the strong $A_{2 g}$-type contribution to vibronic coupling of $v_{4}$. Strong antisymmetric contributions to the Raman tensor of symmetric $A_{1 g}$-type modes have been observed for highly nonplanar metal porphyrins with a symmetric arrangement of peripheral substituents $[63,71]$, thus confirming the above interpretation.

The qualitative, group theoretical analysis presented thus far and the more detailed analysis described in our earlier papers [60] reveal a significant presence of in-plane $B_{1 g^{-}}$ and $B_{2 g}$-type deformations as well as the combined effect of ruffling $\left(B_{1 u}\right)$ and saddling $\left(B_{2 u}\right)$ for ferrocytochrome c. Raman dispersion data for ferricytochrome $c$ are more limited in scope, but they are diagnostic of an above the average rhombic $B_{1}$-deformation which leads to rather large depolarization values for the $v_{4}$-band in the Soret band region $(\rho>0.2)$ [72]. The depolarization ratio of $A_{1 g}$-modes is rather insensitive to $B_{1 g^{-}}$and $B_{2 g}$-type deformations in this spectral region owing to the dominance of $A_{1 g}$-type FranckCondon coupling in the Raman tensor $[19,59]$. A similar observation has been made earlier for myoglobin cyanide [59], which as ferricytochrome c exhibits a hexacoordinated low spin state. A large rhombic distortion in heme proteins with this spin and ligation state is likely to result from static Jahn-Teller distortions which are facilitated by the $E_{g^{-}}$ ground state of the heme iron. The existence of a rather strong rhombic deformation in ferric cytochrome c species is confirmed by EPR data, which allowed for the discovery of two types of ferric cytochrome c proteins: type I with a negligible and type II with a very strong rhombic splitting of the $d_{x z, y z}$ energies [30]. Apparently, horse heart cytochrome $\mathrm{c}$ belongs to the type II category.

The NSD analysis of Shelnutt and coworkers allows a comparison of the magnitude of out-of-plane and in-plane 


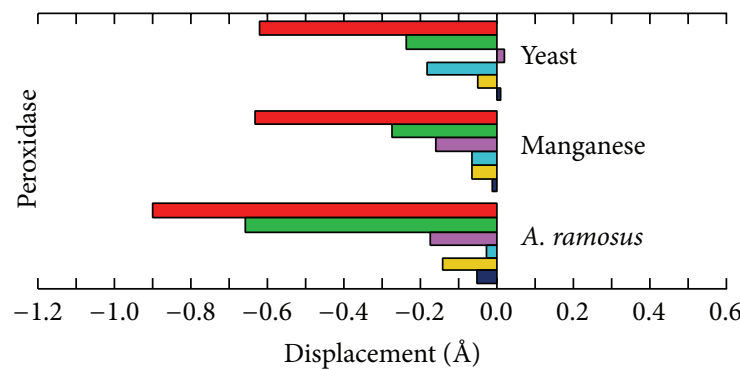

(a)

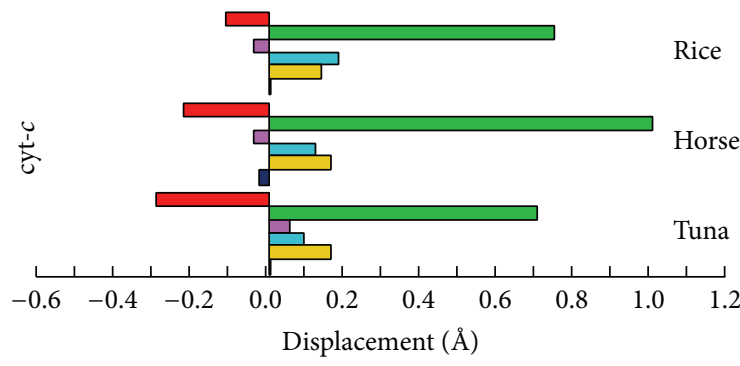

(c)

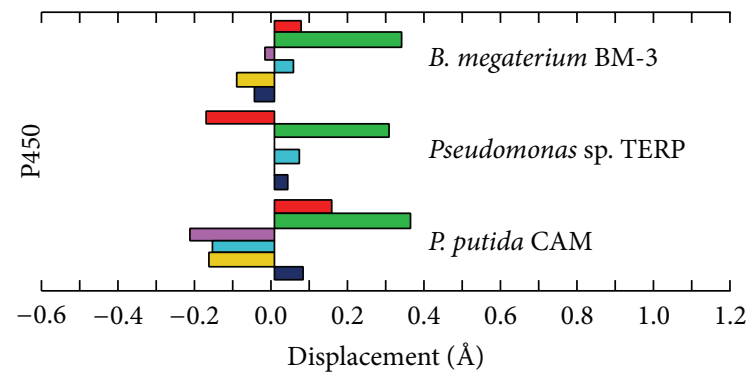

(b)

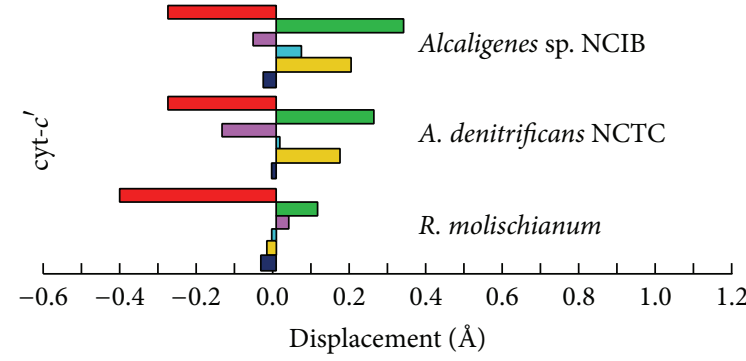

(d)

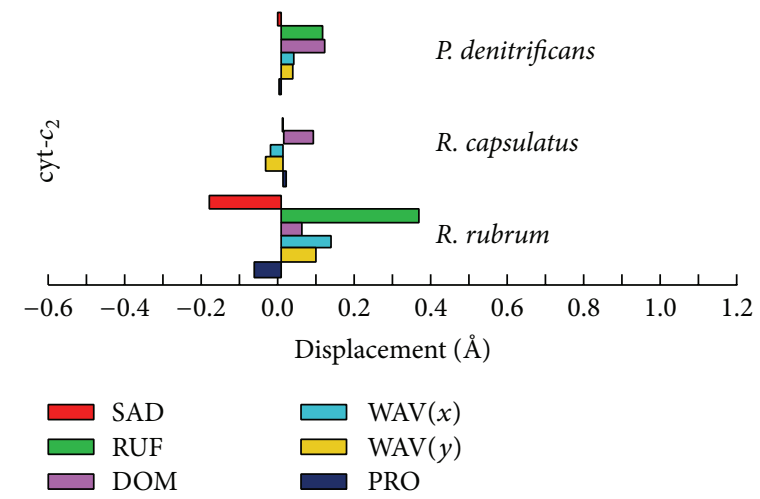

(e)

FIGURE 9: Out-of-plane deformations of the heme group obtained from the NSD analysis of the indicated heme proteins. The color code for the different deformations is displayed at the bottom of the figure. Technical details of the NSD analysis and the pdb-structures used for the analysis can be found in [32], from where this picture was taken and modified.

deformations. While the former can reach $1.0 \AA$ (Figure 12), the latter were found to be in the range between 0.05 and $0.2 \AA$ [32].

Another strategy to analyze out-of-plane deformations of metalloporphyrins has been developed in our group based on measurements of Raman bands assignable to out-of-plane vibrations. They would all be forbidden if the porphyrin (heme) was planar, since modes of ungerade symmetry cannot mix states of $E_{u}$-state. However, in the presence of outof-plane deformations, the vibronic coupling matrix element reads as

$$
c_{e s}^{\Gamma}=\left\langle e\left|\sum_{j} \frac{\partial V^{\Gamma_{j}}}{\partial Q^{\Gamma_{r}}}\right| s\right\rangle Q_{01}^{\Gamma_{r}}=\left\langle e\left|\sum_{j} \frac{\partial^{2} \widehat{H}_{e l}}{\partial Q^{\Gamma_{r}} \partial Q^{\Gamma_{j}}}\right| s\right\rangle Q_{01}^{\Gamma_{r}},
$$

where $\Gamma_{j}$ is the $\mathrm{D}_{4 \mathrm{~h}}$-symmetry of the out-of-plane deformation. To illustrate the meaning of (5) consider a mode of $B_{2 u^{-}}$ symmetry. In the presence of a $B_{2 u}$-deformation (ruffling), corresponding matrix elements in (5) transform like $\Gamma_{j} \otimes \Gamma_{r}=$ $B_{2 u} \otimes B_{2 u}=A_{1 g}$, indicating that the out-of-plane mode becomes Franck-Condon active with B-band excitation. This is exactly what one obtains. We reported a thorough analysis of the intensities and depolarization ratios of several out-ofplane modes of ferrocytochrome $\mathrm{c}$ derivatives (horse heart, chicken, and yeast) and found major differences between the degrees of heme ruffling in these three cytochromes [73]. A comparison with out-of-plane deformation derived from Xray data revealed stronger doming and propellering deformations for the respective proteins in solution. Interestingly, this could be reproduced by molecular dynamics simulations. This suggests some subtle differences between the structures of heme groups of proteins in solution and in single crystals.

Thus far we have based our discussion on the assumption that the depolarization ratio dispersion solely reflects deformations of the ground state. Strictly speaking, this is an oversimplification. As a matter of fact, the matrix elements 
described by (2) reflect differences between the equilibrium coordinates of the excited state and the ground state (if $e=$ $s)$ or between different excited states $(e \neq s)$. However, by inserting (3) and (4) into (2), we obtain

$$
\begin{aligned}
c_{e s}^{\Gamma_{r}}=\langle e| \frac{\partial \widehat{H}_{e l}}{\partial Q^{\Gamma_{r}}}+\sum_{j} \frac{\partial^{2} H_{e l}^{\Gamma_{j}}}{\partial Q^{\Gamma_{r}} \partial Q^{\Gamma_{j}}} \delta Q^{\Gamma_{j}} \\
+\sum_{i} \sum_{j} \frac{\partial^{3} H_{e l}^{\Gamma_{j}}}{\partial Q^{\Gamma_{r}} \partial Q^{\Gamma_{i}} \partial Q^{\Gamma_{j}}} \delta Q^{\Gamma_{r}} \delta Q^{\Gamma_{j}}|s\rangle Q_{01}^{\Gamma_{r}},
\end{aligned}
$$

where the $\delta Q$ terms can be moved out of the second and third terms of the electronic matrix element. Hence, we can conclude that $c_{e s}^{\Gamma}$ depends on both, the ground state deformation and structural differences between the ground state and excited states.

There is now ample evidence based on the investigation of a variety of heme proteins that heme deformations are functionally relevant. More than twenty years ago we reported correlations between $\mathrm{pH}$-induced changes of the ligand affinity of $\mathrm{O}_{2}$ and $\mathrm{CO}$ binding to hemoglobin and corresponding variations of $A_{1 g^{-}}$and $B_{1 g}$-type heme deformations [74, 75]. Several experiments on various metal porphyrins revealed an influence of out-of-plane deformations, particularly ruffling, on ligand binding and redox potentials $[76,77]$. Michel et al. used EPR-data of ferric cytochrome $c_{552}$ mutants to show a correlation between heme ruffling $\left(B_{1 u}\right)$, a destabilization of the iron's $d_{x y}$-orbital, and a decrease of the redox potential $E_{m}$-value $[78,79]$. The destabilization of the $d_{x y}$-orbital $\left(B_{2 g}\right)$ is a result of the capability of $B_{1 u}$-type ruffling to mix with the $A_{2 u}(\pi)$ orbital. Based on EPR and NMR investigations of Pseudomonas aeruginosa cytochrome $\mathrm{c}_{551}$ and Nitrosomonas europaea cytochrome $c_{552}$, Zoppelaro et al. proposed a correlation between rhombic $\left(B_{1 g}\right)$ deformations and ruffling $\left(B_{1 u}\right)$ [30]. As shown in a more recent paper from our group, each type of out-of-plane deformation can indeed induce in-plane deformation of the porphyrin macrocycle described by [72]

$$
\Delta_{g}^{\Gamma}=\sum_{\lambda(\Gamma)} \sum_{\Gamma_{i}} \sum_{i} \frac{\left\langle g\left|\partial^{3} \widehat{H}_{e l} / \partial Q_{\lambda}^{\Gamma} \partial^{2} Q_{i}^{\Gamma_{i}}\right| g\right\rangle}{\left(\Omega_{\lambda}^{\Gamma}\right)^{2}}\left(\delta Q_{i}^{\Gamma_{i}}\right)^{2},
$$

where $\Delta_{\lambda}^{\Gamma}$ is the total ground state deformation of symmetry $\Gamma$. The first sum runs over all vibrations exhibiting the symmetry $\Gamma ; \delta Q_{i}^{\Gamma_{i}}$ denotes any normal deformation of symmetry $\Gamma_{i}$ of the $\mathrm{D}_{4 \mathrm{~h}}$ point group. Obviously, the largest deformation (ruffling in the case of cytochrome c) is capable of inducing all types of in-plane deformations of the ground state.

\subsection{Probing the Electric Field of the Protein in the Heme Plane.} The deformations discussed thus far are generally assigned to noncovalent heme-protein interactions. Ligand-heme interactions are generally asymmetric and can promote in-plane and out-of-plane deformations [11, 76]. In cytochrome c, the influence of peripheral substituents cannot be neglected. The thioether bridges between heme and CxyC motif are a dominant cause for ruffling, which can be modified by the axial ligands $[32,68]$. Propionic acid substituents are hydrogen bonded to the protein matrix; a change of the latter can induce additional deformations $\left(B_{2 g}\right.$ in the case of a Y57F mutant), as shown in one of our more recent studies [41]. In addition to these contact interactions, the influence of the electric field on the heme pocket has to be taken into account. Fluorescence hole burning experiments on heme proteins with $\mathrm{Zn}$-substituted heme groups revealed electric field strengths in the range of $10^{7} / \mathrm{cm}$. Theoretical calculations based on the solution of Laplace equations yielded values in the same order of magnitude [36]. Manas et al. argued on theoretical grounds that this internal electric field can cause a splitting of both the $Q$ - and $B$-band, if the field vector does not exactly bisect the two transition dipole moments of the twofold degenerate electronic transitions [35]. Band splitting has indeed been observed for the Q-band of ferrocytochrome c derivatives at cryogenic temperatures [33-35, 61, 80]. It is generally not detectable in the $B$-band region, owing to the large band width of the overlapping $B_{x}$ - and $B_{y}$-band. However, absorption spectra of $\mathrm{Zn}$-substituted cytochrome c, in which the $B$-band is narrower due to the absence of nonradiative decay via the metal states, seem to indicate such a splitting [35].

More direct evidence for $B$-band splitting was recently provided by us $[37,81]$ based on a comparison of visible $C D$ and absorption profiles in the Soret band region. Figure 10 depicts CD and absorption in the Soret band region of ferriand ferrocytochrome $c$. The CD spectrum of the oxidized protein shows a clear couplet, whereas the corresponding spectrum of the reduced species is somewhat more complex. In both cases the CD spectra do not resemble the respective absorption profile. This indicates an underlying band structure, which results from band splitting due to the influence of an internal electric field via a quadratic Stark effect. If one assumes the field to be homogeneous (which is a somewhat crude approximation), second order perturbation theory yields the following splitting of the $B$-state energies [37]:

$$
\begin{aligned}
& E_{B x}=E_{B x}^{0}+\frac{\left\langle B_{x}|\vec{\mu}| g\right\rangle^{2}}{E_{g}^{0}-E_{B x}^{0}} E^{2} \cos ^{2} \theta \\
& E_{B y}=E_{B y}^{0}+\frac{\left\langle B_{y}|\vec{\mu}| g\right\rangle^{2}}{E_{g}^{0}-E_{B y}^{0}} E^{2} \sin ^{2} \theta,
\end{aligned}
$$

where $E$ is the electric field strength and $\theta$ is the angle between the electric field and one of the N-Fe-N axis of the heme. $E_{g}^{0}$ is the ground state energy of the unperturbed heme. $E_{B x}^{0}=$ $E_{B y}^{0}$ denote the energies of the unperturbed excited state. If $\theta \neq \pi / 4$, the electric fields shift the $x$ - and $y$-components by a different amount, thus causing a splitting of the $B$-band. This splitting can be significant owing to the strong transition dipole moment of the $B$-state transition. This splitting can lead to a CD couplet if the two components carry rotational strength of opposite sign, which is the case in both redox states of cytochrome c.

The solid lines in Figure 10 resulted from a comprehensive analysis which also took vibronic perturbations into consideration. $B_{1 g}$-perturbations, for instance, can either increase 


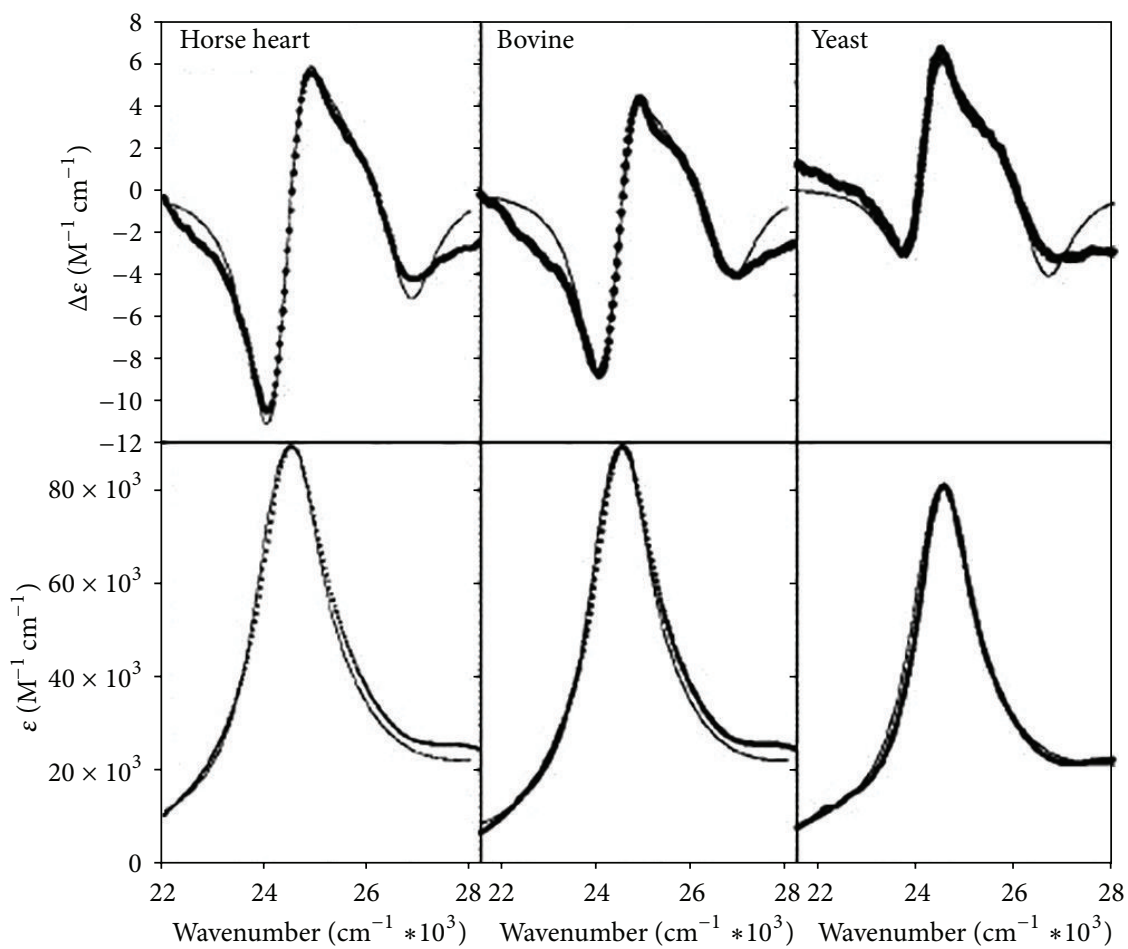

(a)

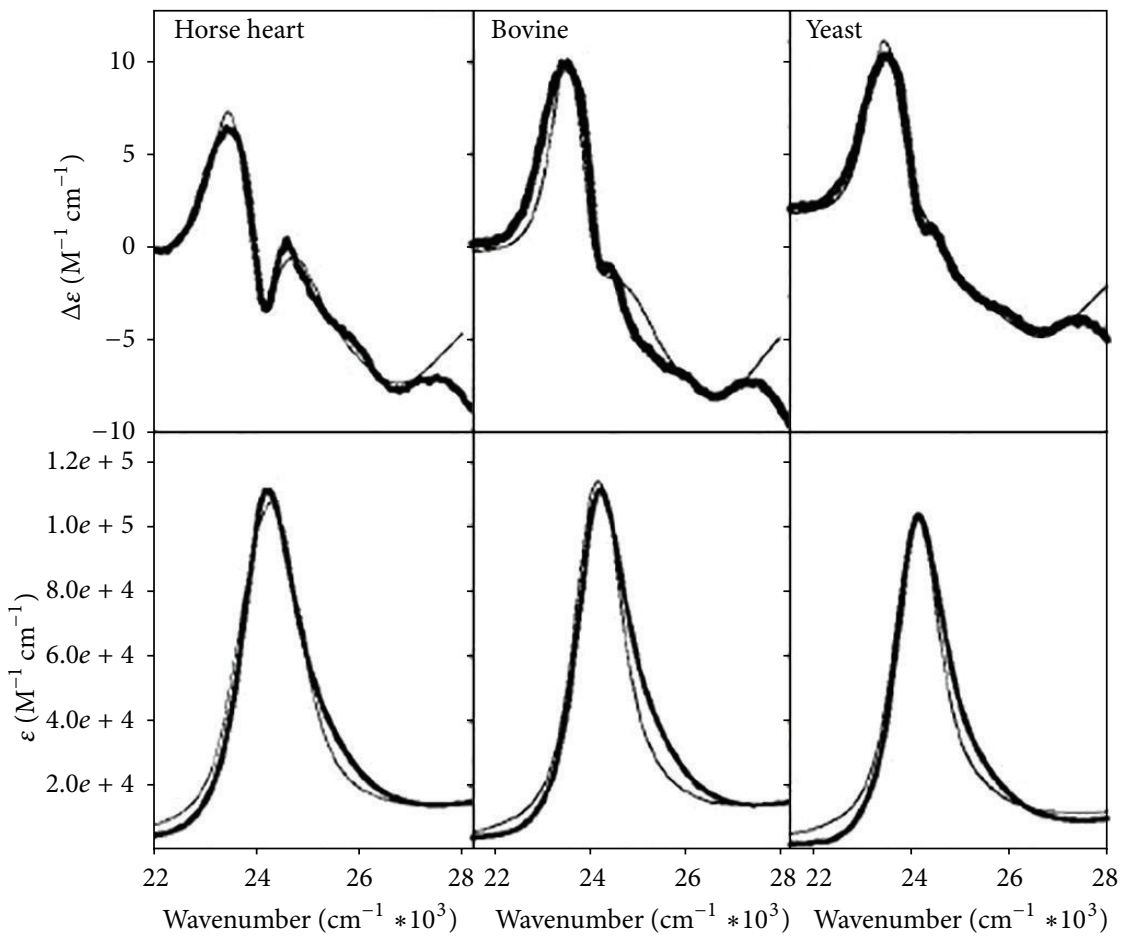

(b)

FIGURE 10: Circular dichroism and absorption spectra in the Soret $(B)$ band region of horse heart ferri- (upper figures) and ferrocytochrome c (lower figures). The solid lines in these figures result from a global vibronic analysis described by Schweitzer-Stenner [37], from where this figure was taken and modified. 
or decrease electric field induced coupling depending on the sign of the coupling matrix elements $[37,59]$. While the Stark effect does not alter the individual band profile of $B_{x}$ and $B_{y}$, vibronic $B_{1 g}$-perturbations can have a major impact in this regard, since they enter the Franck-Condon coupling terms of $B_{x}$ and $B_{y}$ with different signs. As a consequence, the vibronic progression of one component is enhanced, while that of the other one gets substantially diminished [82]. Considering these vibronic contributions is essential for simulating the CD-band profiles of both cytochrome $\mathrm{c}$ species correctly. For myoglobin cyanide, strong $B_{1 g}$-perturbations lead to the dispersion of the absorption polarization of single $\mathrm{MbCN}$ crystals [59].

The electric field induced and vibronic splitting of the $B$ bands of ferri- and ferrocytochrome $\mathrm{c}$ as obtained from the above discussed analysis of Schweitzer-Stenner are listed in Table 1. The electric field values are in good agreement with results from the earlier mentioned hole burning experiments. The difference between the electric field strengths in the two oxidation states accounts for a substantial fraction of the protein's redox potential. It should be noted that the electric field strength and the difference between the oxidation states are less in yeast cytochrome c [59].

Some common misconceptions in interpreting spectroscopic data of heme proteins deserve to be briefly discussed here. It is well established that a substantial part of the rotational strength of heme transitions is induced by dipoledipole coupling with chromophores in the protein (aromatic side chains and protein backbone) $[83,84]$. Another contribution results from nonplanar deformations which can destroy the inversion center of the heme [85]. In this context the two components of the CD couplet displayed in the Soret region of ferricytochrome $\mathrm{c}$ are interpreted as resulting from the coupling of the heme with different amino acid residues in the heme pocket without wasting any thoughts about the physical origin of the couplet. A prominent example is the effect of the reduction of the negative component of the couplet observed in CD spectra of cytochromes in which a single phenylalanine residue in the $\Omega$-loop is replaced by a nonaromatic residue $[86,87]$. In yeast cytochrome $c$ this residue is at position 87 , while horse heart cytochrome $c$ contains it at position 82. Pielak et al. measured the Soret CD of yeast cytochrome $\mathrm{c}$ mutants in which F87 was replaced by other aromatic (Y) and nonaromatic residues (G,S) [86]. In each case the negative component of the couplet disappeared. Since the negative component in the native CD spectrum is considered as an indicator of the native state, its absence is interpreted as a conformational change into a less folded state. This notion is corroborated by the negative component's disappearance in ferricytochrome $c$ spectra measured at extreme $\mathrm{pH}$, high temperatures [88], and high denaturant concentrations [89]. However, the same effect does not imply the same cause. First of all, a sole inspection of the CD spectrum does not reveal all necessary facts; one has to compare CD with their corresponding absorption spectra $[81,82]$. The absence of the negative couplet in the CD spectra of the above discussed F87 mutants of yeast ferricytochrome c could just reflect the elimination of one important mode of transition dipole coupling between protein and heme,
TABLE 1: Listing of B-band spectral parameters of ferri- and ferrocytochrome derivatives inferred from a vibronic analysis of the respective $C D$ and absorption band profile: $\Delta E_{\text {elect }}^{B}$ : electronic (Stark) splitting, $\Delta E_{\text {vib }}^{B}$ : vibronic splitting, $\widetilde{v}_{B}$ : wavenumber associated the average transition energy of the split B-band, and $\Gamma_{B}$ and $\sigma_{B}$ : Lorentzian and Gaussian halfwidths of the Voigtian B-band profile. Taken from [37].

(a) Ferricytochrome c

\begin{tabular}{lcc}
\hline & Horse heart (bovine) & Yeast \\
\hline$\Delta E_{\text {elect }}^{B}\left[\mathrm{~cm}^{-1}\right]$ & -505 & -380 \\
$\Delta E_{\text {elect }}^{B}+\Delta E_{\text {vib }}^{B}\left[\mathrm{~cm}^{-1}\right]$ & -546 & -392 \\
$\widetilde{v}_{B}\left[\mathrm{~cm}^{-1}\right]$ & 24500 & 24500 \\
$\Gamma_{B}\left[\mathrm{~cm}^{-1}\right]$ & 600 & 600 \\
$\sigma_{B}\left[\mathrm{~cm}^{-1}\right]$ & 100 & 100 \\
\hline
\end{tabular}

(b) Ferrocytochrome

\begin{tabular}{lccc}
\hline & Horse heart & Bovine & Yeast \\
\hline$\Delta E_{\text {elect }}^{B}\left[\mathrm{~cm}^{-1}\right]$ & 126 & 186 & 217 \\
$\Delta E_{\text {elect }}^{B}+\Delta E_{\mathrm{vib}}^{B}\left[\mathrm{~cm}^{-1}\right]$ & 516 & 311 & 242 \\
$\widetilde{v}_{B}\left[\mathrm{~cm}^{-1}\right]$ & 24300 & 23900 & 23800 \\
$\Gamma_{B}\left[\mathrm{~cm}^{-1}\right]$ & 330 & 330 & 330 \\
$\sigma_{B}\left[\mathrm{~cm}^{-1}\right]$ & 237 & 237 & 237 \\
\hline
\end{tabular}

which contributes to the negative rotational strength of one of the $B$-state transitions. In this case the substitution of F87 by a nonaromatic residue would just reduce the negative Cotton band without shifting the absorption band and the positive maximum. If this mutation causes structural changes (e.g., involving a rupture of the $\mathrm{Fe}^{3+}-\mathrm{M} 80$ coordination) one would expect a blueshift of the absorption spectrum and a reduced noncoincidence between absorption and positive Cotton band. As will be described in more detail in the next chapter that is what is generally observed for partially unfolded states of ferricytochrome $\mathrm{c}$ in solution.

The above discussed Stark splitting is much less pronounced in the Q-band region, owing to the much weaker dipole moment of the Q-state transition [35, 37]. However, optical absorption measurements on ferrocytochrome $c$ at cryogenic temperatures revealed a $\sim 100-120 \mathrm{~cm}^{-1}$ splitting of the $Q$-band with intensities for the $Q_{x}$ and $Q_{y}$ components $[35,61,90]$. Such an intensity redistribution cannot be explained with a quadratic Stark effect. We invoked instead a $B_{1 g}$-type electronic perturbation combined with vibronic contributions, which can be shown to indeed cause a splitting and a redistribution of oscillator strength [61]. This perturbation (and thus the splitting) is larger in horse heart than in yeast cytochrome c (Table 1). We measured the cryogenic Qband spectra of a series of yeast ferrocytochrome c mutants [91] for which Blouin and Wallace had determined the enthalpic and entropic contributions to the redox potential [40]. We could show that the $B_{1 g}$ perturbations inferred from the optical spectra correlate nicely with the square of the enthalpic contribution to the redox potential. This again underscores the functional relevance of heme perturbations of cytochrome c. 


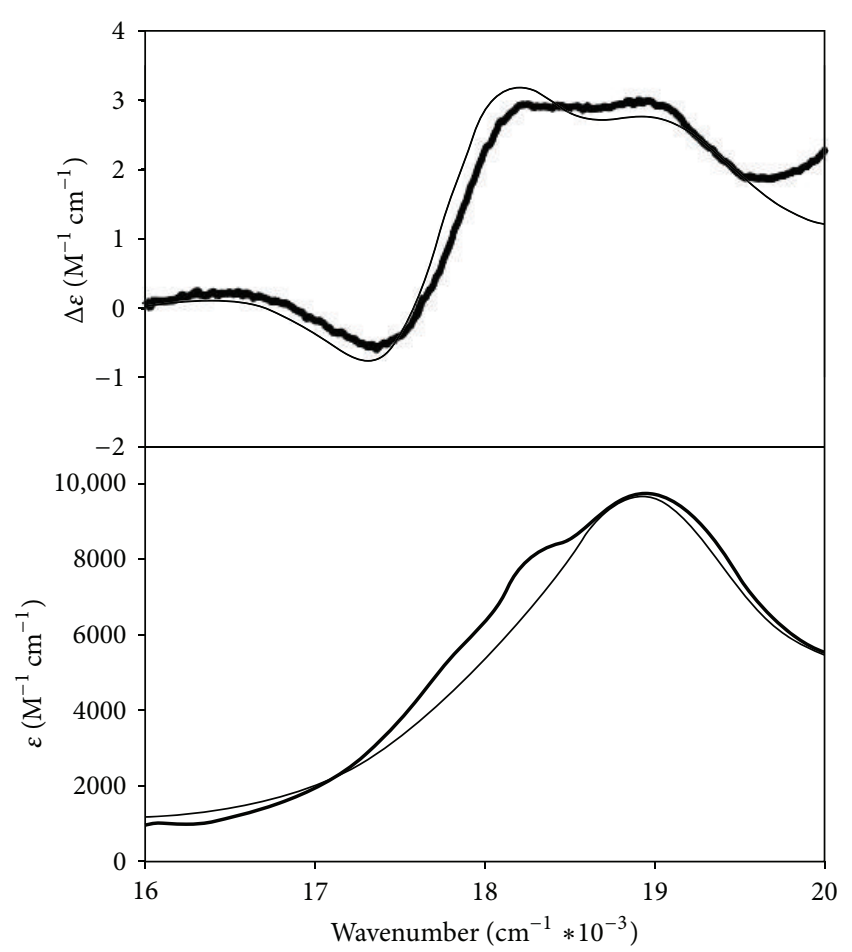

FIGURE 11: Circular dichroism and absorption spectra in the Q-band region of horse heart ferricytochrome $c$. The solid lines resulted from a global vibronic analysis of the spectra described by SchweitzerStenner [37], from where this figure was taken and modified.

The Q-band region of ferricytochrome c is more difficult to analyze because the rather broad bands associated with individual transitions into $Q_{x}$ and $Q_{y}$ states do not allow a separation even at cryogenic temperatures. However a comparison of $\mathrm{CD}$ and absorption spectra reveals a noncoincidence at the $Q_{0}$-position which indicates band splitting (Figure 11) [37, 81]. Moreover, the CD spectra show a much better resolution of the vibronic $Q_{v}$-band which facilitates the analysis further. The Q-band splitting in ferricytochrome c is again assignable to an electronic $B_{1 g}$-type perturbation.

Taken together, this chapter describes a variety of experimental techniques which can be employed to explore inplane and out-of-plane deformation of the heme group in cytochrome c. The same methods can of course be applied to other heme proteins. Out-of-plane deformations have attracted more attention in the last fifteen years owing to the fact that they can be easily inferred from the proteins' crystal structures. However, in-plane deformations lowering the symmetry of the crystal field probed by the heme iron should be of similar functional relevance. The same can be said about the internal electric field in the heme plane.

\section{Folding and Unfolding of Cytochrome $\mathrm{c}$ in Solution}

3.1. Thermal and pH-Induced Unfolding of Cytochrome c. Over the last twenty years cytochrome $c$ has emerged as one of the most prominent model systems for studying the folding and unfolding of proteins. Most of the research has thereby focused on ferricytochrome $\mathrm{c}$ because it is much less stable than the reduced state of the protein. To unfold the latter, temperatures above $100^{\circ} \mathrm{C}$ or extreme $\mathrm{pH}$ values below 3 and above 12 are required [92-96]. Ferricytochrome c, however, adopts a variety of states, if the $\mathrm{pH}$ is changed between 1 and 12 and begins to thermally unfold above $50^{\circ} \mathrm{C}$ [20]. The reason for the oxidized protein's lesser stability lies (a) in the reduced strength of the $\mathrm{Fe}^{3+}$-M80 coordination [97], which is pivotal for maintaining the protein's native structure [98], and (b) to a minor extent in larger conformational flexibility $[99,100]$ and solvent accessibility of the protein's structure in the oxidized state $[101,102]$.

$\mathrm{pH}$-induced unfolding of ferricytochrome $\mathrm{c}$ has a long tradition of research. It was investigated even prior to the classical folding unfolding/refolding experiment of Anfinsen [104]. Nearly 70 years ago Theorell and Åkesson discovered five different titration states which they denoted by the Roman numerals I to V [105]. Since then, numerous studies have been performed to characterize these states, the result of which is summarized by the scheme shown in Figure 12 . States I and II are known to be partially unfolded and are populated at acidic $\mathrm{pH}[94,106-108]$. The actual state of I depends heavily on ionic strength. It is highly disordered with reduced secondary structure content at low ionic strength but gains secondary structure upon addition of $\mathrm{NaCl}$, owing to the shielding of repulsive Coulomb interactions between positively charged groups by chloride ions $[109,110]$. This A-state unfolds cooperatively with increasing temperature. Different spin states of the heme iron can coexist in state II, depending on solution conditions [110]. State III is generally considered as the most folded state, often referred to as the "native" state. States IV and V are the alkaline states in which M80 is replaced by lysine residues and hydroxyl ions [111-113]. Each of them can be divided into substates with different axial ligands.

The Theorell-Åkesson scheme was recently augmented by us, when we reported the existence of a state III* which is an intermediate between III and IV [103]. It is similar to III in that it still contains a (most likely weakened) Fe-M80 bond. It is detectable only at low ionic strength, explaining why it is often missed in spectroscopic experiments. Figure 13 shows the integrated intensities of $695 \mathrm{~nm}$ subbands (their origin will be discussed below) as a function of $\mathrm{pH}$. The data clearly reveal two different phases which merge into a single one at high phosphate ion concentration. Cherney and Bowler assigned the state to the deprotonation of one of the propionic acid substituents of the heme group, which is known to exhibit a very high $\mathrm{pK}$-value [114]. A similar state called $\mathrm{III}_{3.5}$ was observed from site specific IR-experiments of Weinkam et al., which as III* is populated at $\mathrm{pH} 9$ [115]. These authors argued that $\mathrm{III}_{3.5}$ does not exhibit a $695 \mathrm{~nm}$ band in the optical spectrum, which is indicative of the integrity of the Fe-M80 linkage. Our data, however, show that a weaker $695 \mathrm{~nm}$ band is still present at $\mathrm{pH} 9$ and low ionic strength. This contradiction could be resolved by assuming the coexistence of two different intermediates III $^{*}$, one with and one without M80 ligation. At low ionic strength the former is more populated 


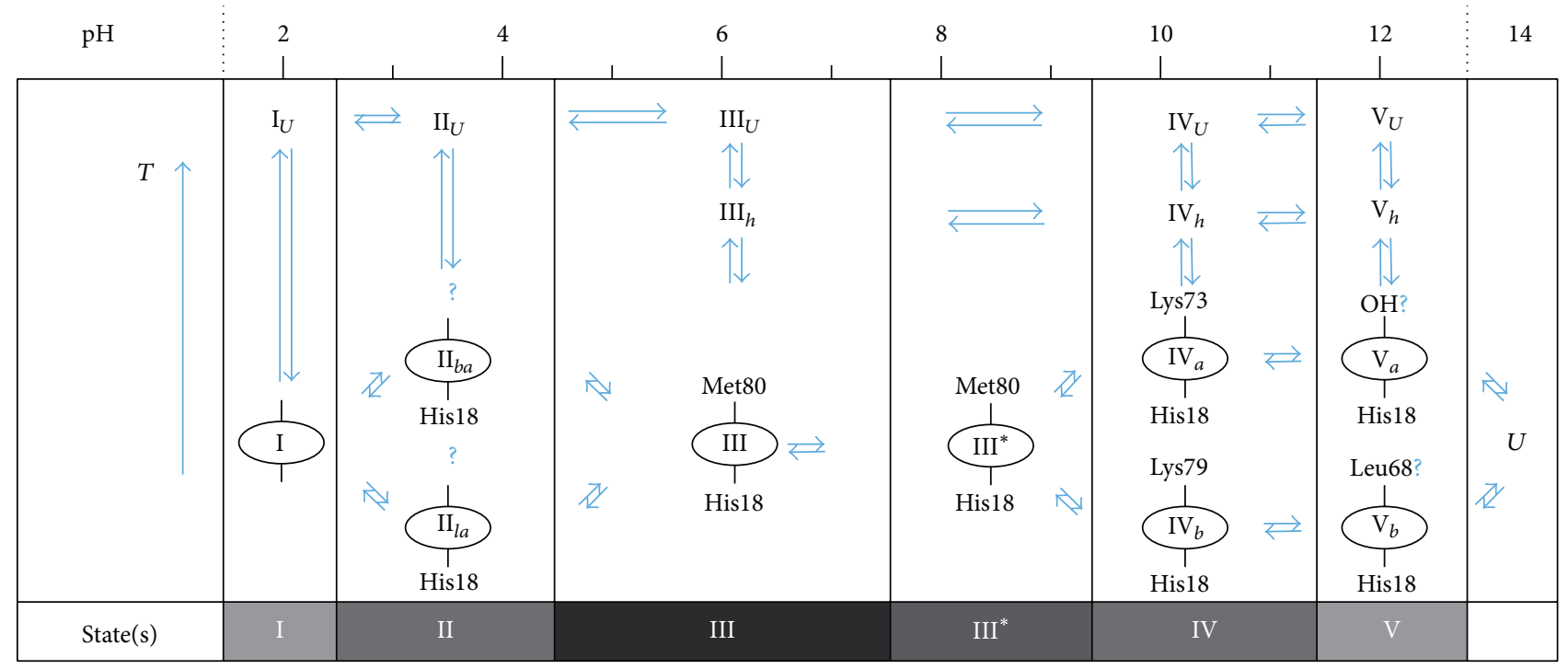

FIGURE 12: Schematic representation of the different protonation states of ferricytochrome $\mathrm{c}$ with the corresponding ligation states of the heme iron. In addition to the canonical Theorell-Åkesson states I, II, III, IV, and V the scheme displays a recently discovered intermediate III* and the different isomer of states II, IV, and V. Produced by Dr. Jonathan Soffer in the author's research group.

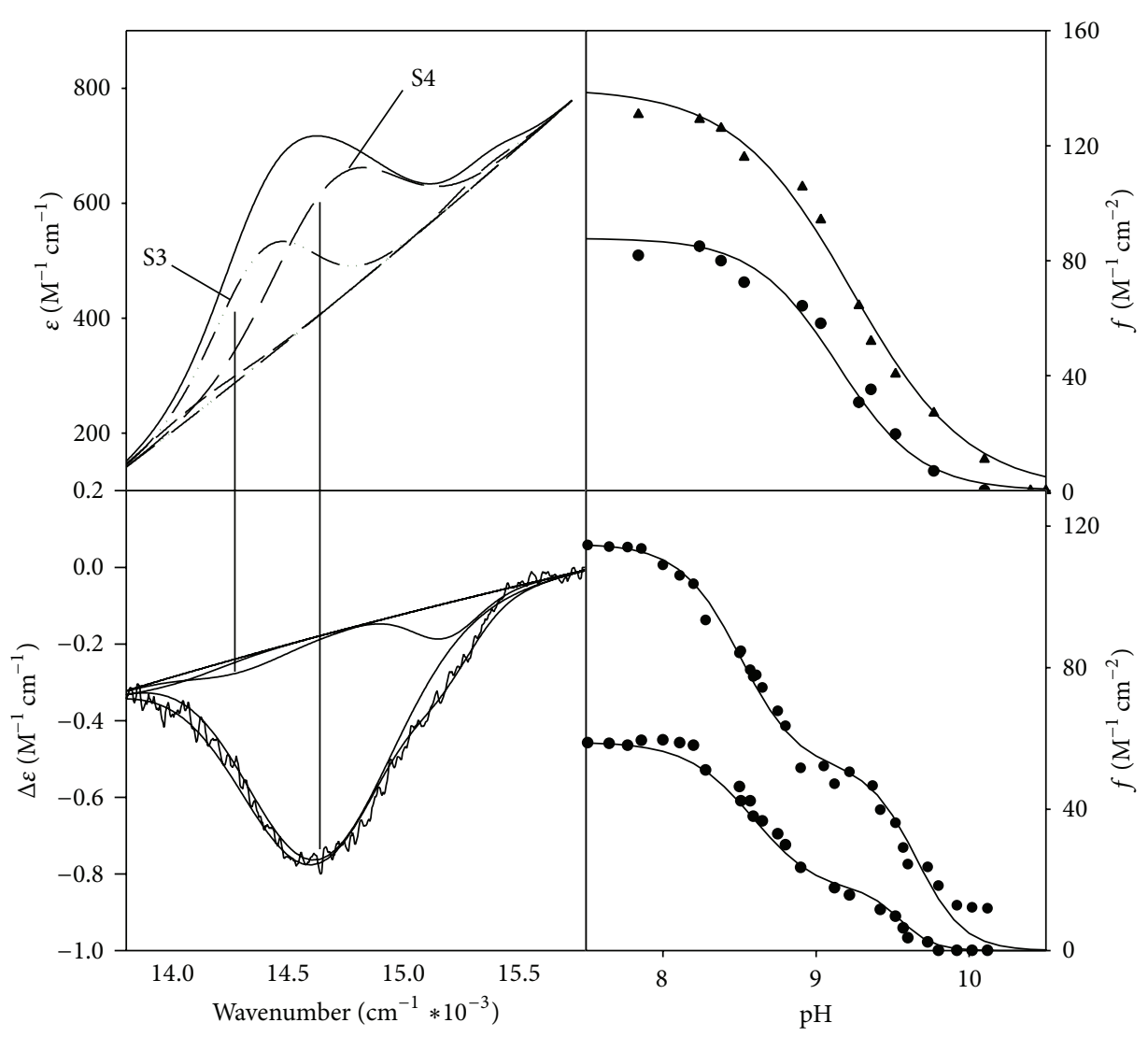

FIGURE 13: Integrated intensities of two subbands of the $695 \mathrm{~nm}$ charge transfer band of horse heart ferricytochrome c plotted as a function of $\mathrm{pH}$. The decomposition of the absorption and CD band is shown in the left. The solid lines in the plots resulted from the fit of a titration model outlined by Verbaro et al. [103], from where we took the figure with permission. 
while the latter is predominant at high ionic strength, at which the experiments of Weinkam et al. were performed.

The transitions III $\Rightarrow$ IV and III $\Rightarrow$ II can be considered as the prime $\mathrm{pH}$-induced unfolding transitions. It is therefore no surprise that they were particularly intensively studied. III $\Rightarrow$ IV is called the alkaline transition which involves the above intermediates III* and/or $\mathrm{III}_{3.5}$. The disappearance of the $695 \mathrm{~nm}$ band in IV indicated early that M80 is replaced by another ligand in this state [116]. Optical and resonance Raman studies on yeast cytochrome $\mathrm{c}$ mutants by Mauck, Hildebrandt and their respective coworkers revealed the coexistence of two state IV isomers with K73 and K79 (Figure 12) [112]. Since these isomers are in thermodynamic equilibrium, they cannot be responsible for the two phases of the III $\Rightarrow$ IV transition observed by Verbaro et al. [103] and Weinkam et al. [115]. In horse heart cytochrome c, the existence of another lysine (K72) complicates the picture further in that it can also bind to the heme [117]. In all these histidine-lysine ligand complexes of the heme, the reduction potential is substantially reduced, thus stabilizing the oxidized state $[113,118]$.

One would be tempted to assign the III $\Rightarrow$ III $^{*} \Rightarrow$ IV transition to lysine deprotonation. However, the intrinsic $\mathrm{pK}$ of lysine is at 11.5, which is way too high to explain the observed titrations. Very early, Davis et al., based on kinetic experiments, showed that the alkaline transition involves a kinetic intermediate; the protonation process is followed by a conformational change which lowers the effective $\mathrm{pK}$ value(s) of the transition [116]. Our analysis showed that both III $\Leftrightarrow$ III $^{*}$ and III $^{*} \Leftrightarrow$ IV equilibria are describable by a Hill-type equation, which indicates the involvement of more than a single proton. As indicated above, Cherney and Bowler suggested that one of the propionic acid groups of the heme is a candidate [114].

The effective pK-value of the alkaline transition depends on the ionic strength of the solution. Battistuzzi et al. found that the pK-value increases monotonically with ionic strength $[118,119]$. However, our data reveal that this is true only for the pK-value of the III $\Leftrightarrow$ III $^{*}$; the pK-value of III $^{*} \Leftrightarrow$ IV decreases with increasing ionic strength [103]. It is this combined effect which obfuscates the identification of III* at high ionic strength. According to Battistuzzi et al., an increase in the temperature decreases the effective $\mathrm{pK}$ of the alkaline transition [119].

Hoang et al. used HD exchange and kinetic studies to further characterize the III $\Leftrightarrow$ IV transition [120]. They found strong evidence for this transition to involve the unfolding of the $\Omega$-loop foldons. Unfolding means here a misligation of the heme. In order to allow ligation by one of the lysines, the $\Omega$-loop has to carry out something like a translational move with respect to the heme group. This view is in agreement with the NMR-based structure that Assfalg et al. reported of two yeast cytochrome $\mathrm{c}$ mutants, K73A and K79A [121]. The measurements were carried out at $\mathrm{pH} 11$ and moderate ionic strength (50 $\mathrm{mM}$ phosphate buffer). It is not clear whether the protein is exclusively in state IV at these conditions. The use of the two mutants avoids complications which would arise from the coexistence of the two state IV isomers. The NMR structure reveals that the most dominant changes occur

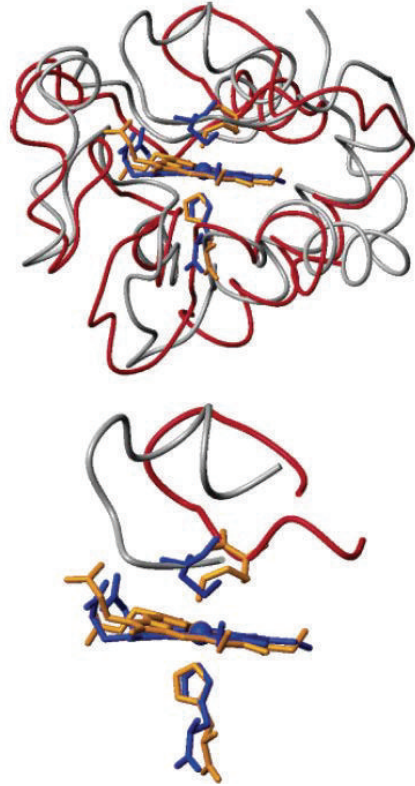

FIGURE 14: Comparison of the structure of alkaline (state IV/V, red) and native (grey) yeast ferricytochrome c derived from twodimensional NMR experiments. The figure was taken from [121] with permission.

indeed in the $\Omega$-loop region. Figure 14 shows a comparison of the structure of the native and the state IV $(\mathrm{V})$ protein reported by Assfalg et al. [121].

Unfortunately, state $\mathrm{V}$ has not yet attracted as much attention of researchers as state IV. The Raman study of Döpner et al. revealed the existence of two V-state isomers, a dominant $\mathrm{V}_{a}$, and a less populated $\mathrm{V}_{b}$ state [112]. The authors managed to extract the Raman spectra of the latter from their data, in which the position of spin marker bands is consistent with $\mathrm{OH}^{-}$being the sixth ligand. The ligand of $\mathrm{V}_{a}$ might still be a lysine. $\mathrm{CD}$ and absorption spectra to be presented below provide further evidence that states IV and V are indeed distinguishable, particularly with respect to thermal unfolding. The acid unfolding of horse heart cytochrome $c$ was first investigated thoroughly by Myer [123]. Their data led them to propose a scheme III $\Leftrightarrow \mathrm{III}_{a} \Leftrightarrow \mathrm{II} \Leftrightarrow \mathrm{I}$, where $\mathrm{III}_{a}$ was described as a III-like intermediate very similar to the III* state detected by Verbaro et al. [103]. The respective pKvalues were reported as 3.6, 2.7, and 1.2 at low ionic strength. They barely change in the presence of salt. The authors suggested that state II is hexacoordinated high spin and state I is pentacoordinated high spin. A somewhat different picture emerged from the kinetic studies of Dyson and Beattle on the same cytochrome $c$ derivative [106]. They found that the protein has lost both axial ligands in state I, which is highly disordered. Two coexisting states were proposed for state II, one with low and the other with a high spin heme. The authors proposed that the former should still exhibit M80 as ligand. However, this seems to be very unlikely, since the $695 \mathrm{~nm}$ band is absent in the $\mathrm{pH}$ range at which state II is populated. Work of Jeng and Englander as well as of Goto et al. revealed a strong influence of salt on the conformational 
mixture at low $\mathrm{pH}[107,124]$. The protein is predominantly molten globule (i.e., with an intact secondary structure) in state II and a statistical coil in state I at low ionic strength. At high ionic strength the statistical coil state I converts to a molten globule, which was later termed A-state $[109,110,125]$. More recently, Battistuzzi et al. used visible absorption and magnetic circular dichroism spectra to probe conformational transition of yeast iso- 1 ferricytochrome $\mathrm{c}$ and three mutants (M80A, M80A/Y67H, and M80A/Y67A) in the acidic region and a low ion concentration [126]. They found that state III of the wild type first converts into a hexacoordinated high spin state, most likely with water as sixth ligand. Below $\mathrm{pH}$ 2.6, a new hexacoordinated high spin state is formed for which the protein is mostly unfolded and the heme is ligated by two water molecules. The replacement of M80 by an alanine produces a hexacoordinated low spin state with $\mathrm{OH}^{-}$as sixth ligand, reminiscent of state $\mathrm{V}_{b}$ [112]. Lowering the $\mathrm{pH}$ causes the population of different high spin states with $\mathrm{H}_{2} \mathrm{O}$ as sixth ligand which differ in terms of the protonation state of one the heme group's propionic acids and the nature of the proximal ligand.

In our laboratory we recently measured the absorption and visible $\mathrm{CD}$ spectra as function of $\mathrm{pH}$ in the absence of salt and at the lowest possible ionic strength [127]. Figure 15 shows the Kuhn anisotropy $\Delta \varepsilon / \varepsilon$-value measured at the wavelength position of the negative maximum of the native state spectrum as a function of $\mathrm{pH}$. These data clearly allow the identification of all protonation states. The data reproduced the finding of Verbaro et al. [103] in that they distinguish between states III* and IV but gave no indication of the existence of the III $_{a}$-state reported by Myer $[123,128]$. States IV and V are clearly distinguishable.

The thermal unfolding of ferricytochrome has been studied for quite some time. Earlier UVCD measurements by Myer and Pande clearly indicate two phases of thermal unfolding at physiological conditions, an observation implying the existence of an unfolding intermediate [128]. The existence of the latter followed also from the observation that the $695 \mathrm{~nm}$ band disappears at temperatures below the onset of protein unfolding. Taler et al., based on the results of NMR experiments, suggested a $\mathrm{pK}$-shift of the alkaline transition with increasing temperature as the source of this intermediate [129]. However, this notion was questioned by Battistuzzi et al. who, from their analysis of the temperature dependence of the alkaline transition, found a nonnative state populated at high temperatures which is clearly distinct from state III [119].

The thermal unfolding of ferricytochrome c was recently investigated further by us using spectroscopic means. First, we measured the $695 \mathrm{~nm}$ band profile as a function of temperature at $\mathrm{pH} 6$ and 7 [130]. We found that the band profile itself is changing at high temperature. This was related to the slightly different partial unfolding of different subconformation of the protein. We had shown earlier that the $695 \mathrm{~nm}$ band can be decomposed into different subbands that should be related to different substates of the Fe-M80 linkage [81]. Our analysis was later confirmed by the results of low temperature CD measurements [131]. As recognized by

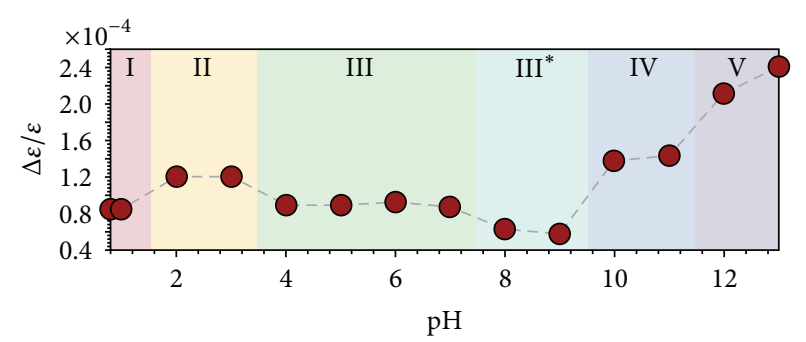

FIGURE 15: Kuhn anisotropy $(\Delta \varepsilon / \varepsilon)$ of $0.5 \mathrm{mM}$ oxidized cyt-c measured at $405 \mathrm{~nm}$ as a function of $\mathrm{pH}$ in $0.1 \mathrm{mM}$ MOPS buffer at $298 \mathrm{~K}\left(25^{\circ} \mathrm{C}\right)$. Taken from [122].

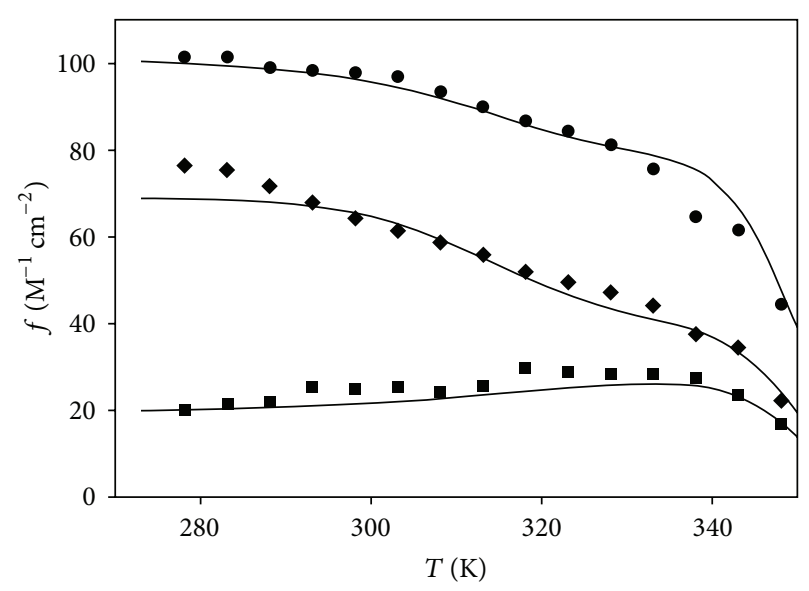

FIGURE 16: Oscillator strengths of the three subbands of the $695 \mathrm{~nm}$ absorption band of bovine heart ferricytochrome $\mathrm{c}$ as function of temperature. Filled circles: S3; triangles: S4; open circles: S2. The solid lines result from a thermodynamic analysis described in the text and by Schweitzer-Stenner et al. [20], from where this figure was taken and modified.

Frauenfelder and coworkers, proteins in different subconformations perform the same function but with different rates [132].

Figure 16 shows the temperature dependence of extinction coefficients measured at three different wavelengths and $\mathrm{pH} 7$, which according to Dragomir et al. correspond to the above subbands [81]. The transition curves are clearly biphasic; one of the curves indicates even the existence of another low temperature phase, which becomes more apparent at acidic $\mathrm{pH}$. These data will be discussed again below, when we present a thermodynamic analysis of different data sets. However, just a visual inspection of the data reveals that an intermediate state is populated at temperatures around $320 \mathrm{~K}$ and that the $\mathrm{Fe}^{3+}-\mathrm{M} 80$ ligation is still intact in this intermediate. It is thus much more reminiscent of the state III* reported by Verbaro et al. [103].

To explore thermal unfolding further, we measured the temperature dependence of the Soret absorption and CD spectra at different $\mathrm{pH}$ values in the neutral and alkaline regions [88]. The selected $\mathrm{pH}$ values correspond to populations of the states III, III*, IV, and V. We used bovine rather than horse heart ferricytochrome $c$, because the former 


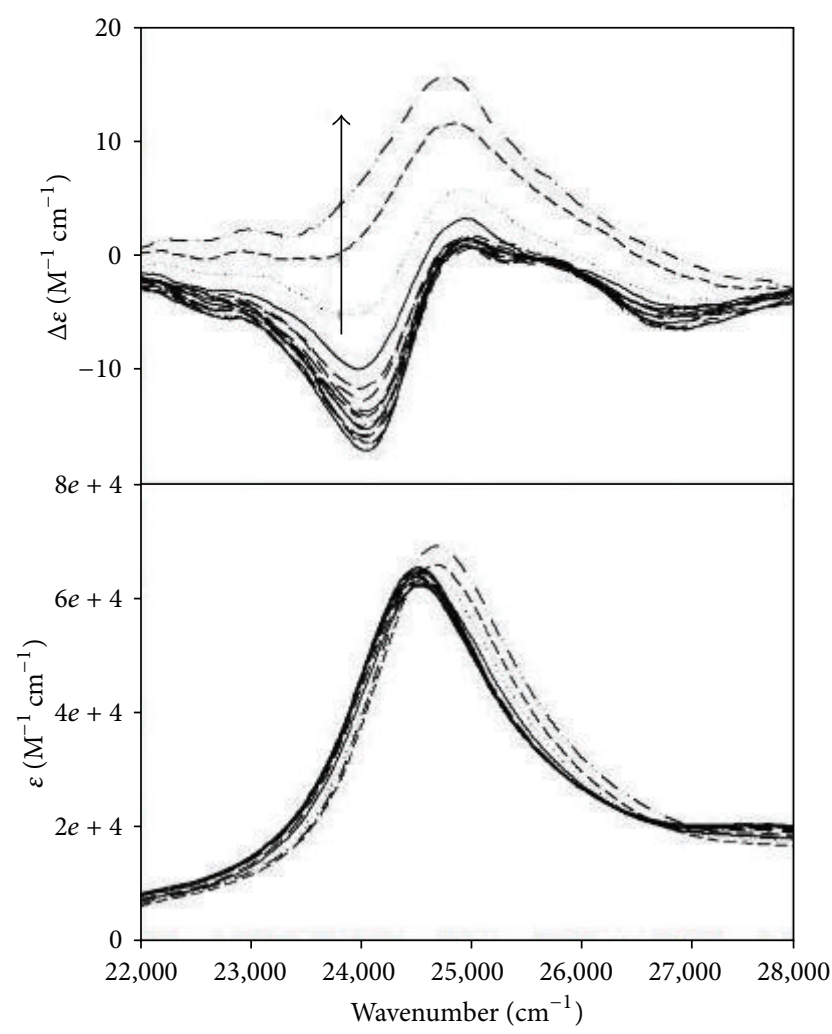

FIgURE 17: CD and absorption spectra of the $B$-band region of oxidized bovine heart cytochrome $\mathrm{c}$ dissolved in a $1 \mathrm{mM}$ MOPS buffer ( $\mathrm{pH} 7$ at room temperature) measured as a function of temperature between $5^{\circ}$ and $90^{\circ} \mathrm{C}$. Arrows indicate changes in temperature. Taken from [20] and modified. The original experimental data are from Hagarman et al. [88].

is less prone to aggregation at high temperatures than the latter. Figure 17 shows the visible $\mathrm{CD}$ and absorption $B$ band spectra measured at $\mathrm{pH}$ 7. The $\mathrm{CD}$ couplet converts into a positive Cotton band with increasing temperature; the corresponding absorption band shifts to the blue, thus minimizing the noncoincidence between absorption and CD. This measurement was deliberately performed with a Tris$\mathrm{HCl}$ buffer, which exhibits a $\mathrm{pH}$ shift with increasing temperature. Thus, the authors avoided any overlap with the alkaline transition. Subsequently, they performed the same measurements with a MOPS buffer, which is very thermostable. These two protocols yielded practically identical results. Figure 18 shows $\Delta \varepsilon$ values measured at the position of the negative and positive maximums of the native protein as a function of temperature. In addition, the temperature dependence of a $\Delta \varepsilon$ of the corresponding UVCD spectrum is displayed. The transition curve obtained from the latter is clearly biphasic; a closer inspection reveals the same for the former. Figure 19 shows the visible Soret CD and absorption profiles measured at $\mathrm{pH} 10.5$ and 11.5 at different temperatures. At the ionic strength conditions used for these measurements $(0.1 \mathrm{M})$, the $\mathrm{pH}$ values correspond to states IV and V. The CD spectra show a positive Cotton band even at room temperature; the rotational strength increases with increasing temperature.

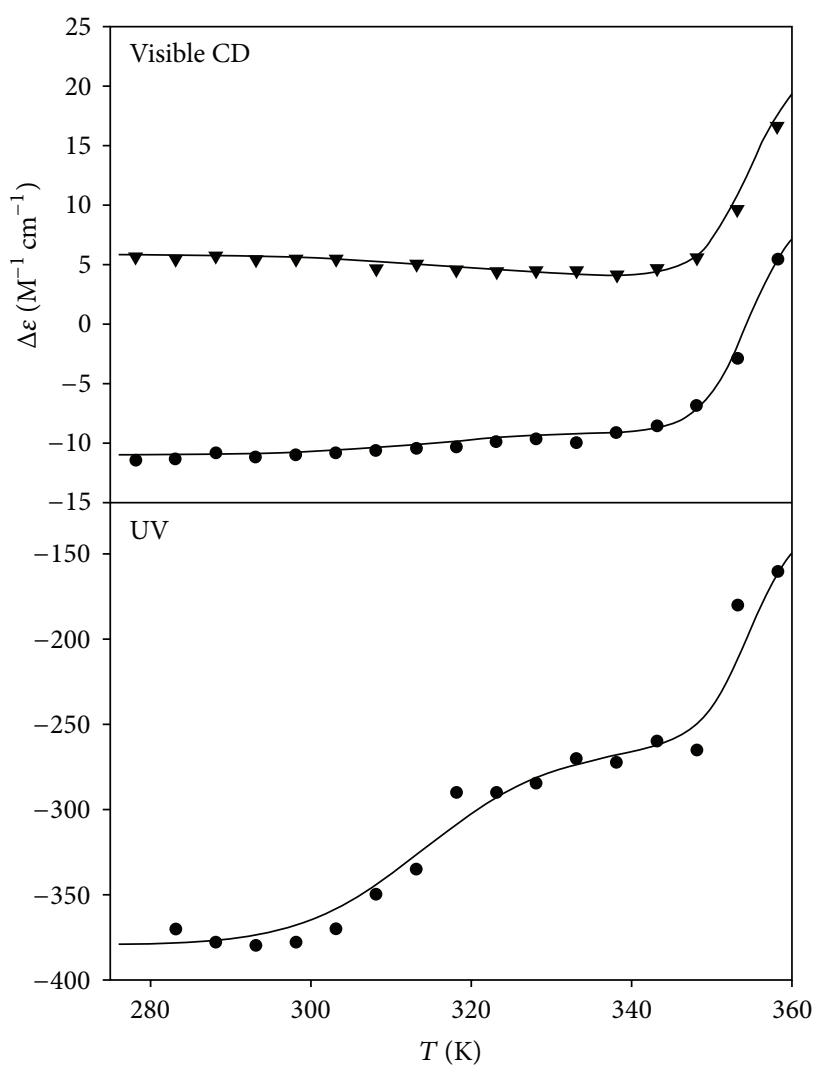

FIGURE 18: $\Delta \varepsilon$ versus temperature of oxidized bovine horse heart cytochrome c between 278 and $363 \mathrm{~K}$. Upper panel: $\Delta \varepsilon$ obtained from the CD spectra in Figure 17 at $24876 \mathrm{~cm}^{-1}$ (triangles) and $24,010 \mathrm{~cm}^{-1}$ (filled circles). Lower panel: $\Delta \varepsilon$ obtained from the corresponding CD spectra at $44964 \mathrm{~cm}^{-1}$. The experimental data were taken from [88]. The solid lines result from fits described in the text. The figure was taken from [20] and modified.

The room temperature spectra for states IV and V are clearly distinct, which is diagnostic of different heme environments. For state V, the increase of the Cotton band with increasing temperature is particularly large. In addition, one observes a rather surprising redshift of the spectrum. At room temperature the CD spectrum is slightly blueshifted compared with the absorption band. This is indicative of some moderate band splitting. The noncoincidence is practically absent at high temperatures. The temperature dependences of $\Delta \varepsilon_{\max }$ (i.e., the dichroism measured at the wavelength, at which the room temperature CD is maximal) are depicted in Figure 20. A two-phase transition is clearly on display for state V; only a closer inspection of the data reveals the population of an intermediate state for the "unfolding" of state IV.

We subjected these data to a global fitting based on the following equation [20]:

$$
\Delta \varepsilon=\frac{\Delta \varepsilon_{n}+\Delta \varepsilon_{i} \cdot e^{-G_{i} / R T}+\Delta \varepsilon_{u} \cdot e^{-G_{u} / R T}}{1+e^{-G_{i} / K T}+e^{-G_{u} / R T}},
$$

where $\Delta \varepsilon_{n}, \Delta \varepsilon_{i}$, and $\Delta \varepsilon_{u}$ are the dichroism values in the native $(n)$, intermediate $(i)$, and $(u)$ unfolded state. $G_{i}$ and $G_{u}$ are 


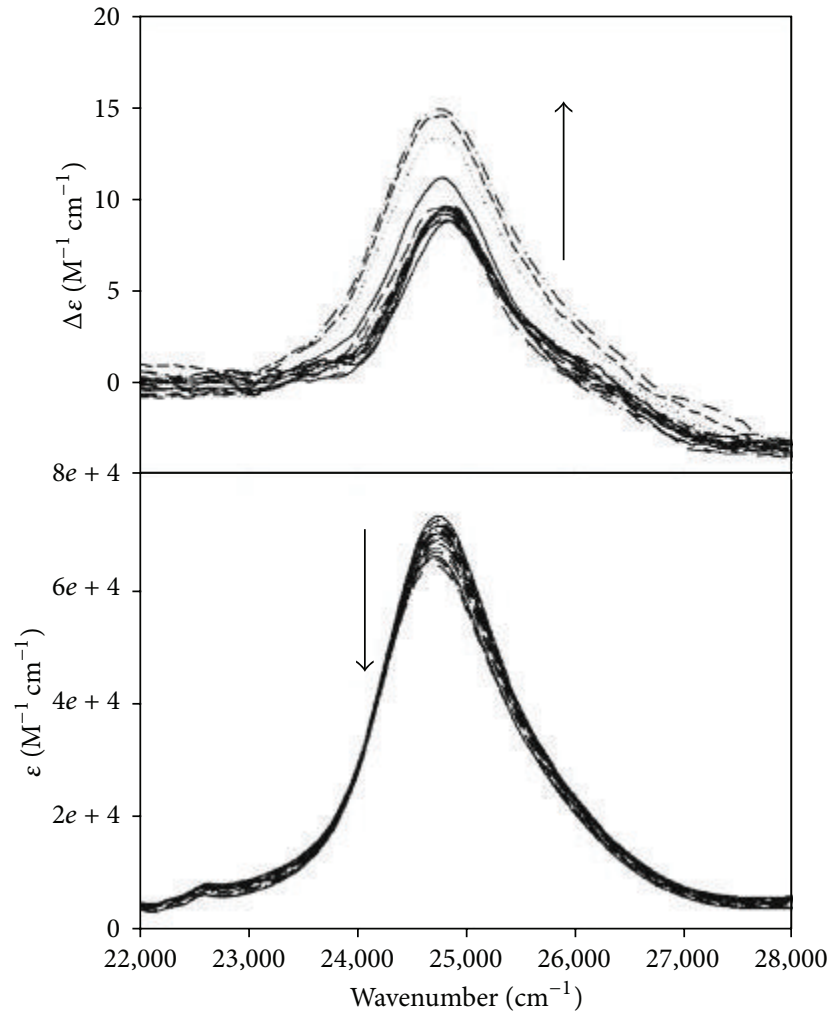

(a)

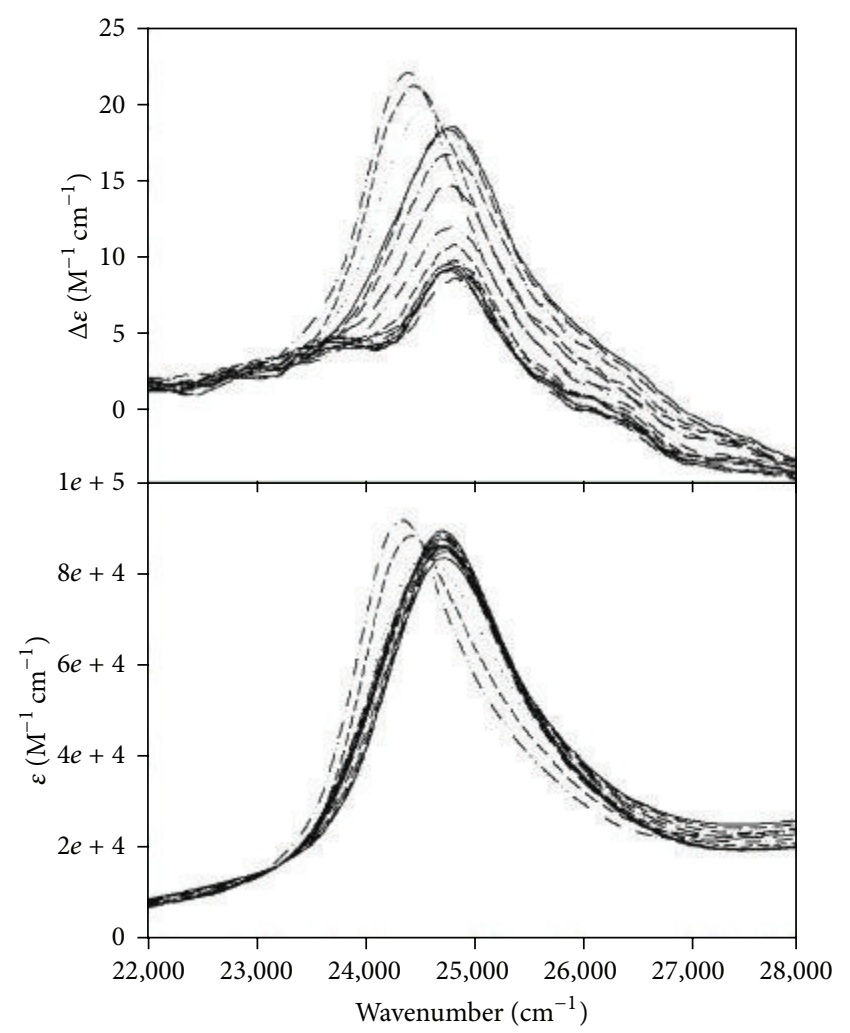

(b)

FIGURE 19: $\mathrm{CD}$ and absorption in the $B$-band region of oxidized bovine cytochrome $\mathrm{c}$ measured as a function of temperature between $5^{\circ}$ and $90^{\circ}$. Left: the measurements were performed with a $\mathrm{pH}$ of 10.5 with a $0.1 \mathrm{M}$ Tris buffer. Right: the measurements were performed with a pH of 11.5 with a $0.1 \mathrm{M}$ Tris buffer. The arrows in the left figure indicate the changes with increasing temperature for both data sets. The original data were obtained from Hagarman et al. [88]; the figure was taken from [20] and modified.

the Gibbs energies of states $i$ and $u$ with respect to $n$, which are written as follows:

$$
\begin{gathered}
G_{i}=H_{i}-T S_{i} \\
G_{u}=H_{u}^{0}+\delta c_{p} \cdot\left(T-T_{i}\right)+T \cdot\left(S_{u}+\delta c_{p} \cdot \ln \left(\frac{T}{T_{u}}\right)\right),
\end{gathered}
$$

where $H_{i}$ and $H_{u}$ indicate enthalpies of the intermediate and unfolded state, $T_{i}$ and $T_{u}$ are the corresponding transition temperatures, $S_{i}$ and $S_{u}$ are the entropies, and $\delta c_{p}$ is the change of the heat capacity associated with the $i \Rightarrow u$ transition. This formalism was used to fit the data in Figures 18 and 20. The obtained thermodynamic parameters are shown in Table 2. We used a modified formalism to globally fit the $695 \mathrm{~nm}$ data in Figure 16 [20], which led to the solid lines shown therein.

Our analysis revealed a rather complex picture of cytochrome $\mathrm{c}$ thermal and $\mathrm{pH}$-induced unfolding. Their data clearly revealed the existence of three nonnative alkaline states, termed III*, IV, and V. Synchrotron UVCD spectra of these states clearly show that the secondary structure is mostly maintained, and the structural changes are predominant on the tertiary level. Each of these states shows
TABLE 2: Gibbs free energy of hydrogen exchange obtained for the indicated protein segments of horse heart ferricytochrome $c$ reported by Krishna et al. [23].

\begin{tabular}{lcc}
\hline Foldon & Identity & $\begin{array}{c}\Delta G_{\mathrm{Hx}} \\
{[\mathrm{kJ} / \mathrm{mol}]}\end{array}$ \\
\hline Blue bihelix & $\begin{array}{c}\mathrm{N} \text { - and C-terminal } \\
\alpha \text {-helices }\end{array}$ & 53.5 \\
Green helix and green loop & $\begin{array}{c}\text { 60's } \alpha \text {-helix and 19-36 } \\
\Omega \text {-loop }\end{array}$ & 40.18 \\
Yellow $\beta$-sheet & 37-39, 58-61 antiparallel & 30.71 \\
Red loop & $\beta$-strands & 26.14 \\
Infrared loop & $71-85 \Omega$-loop & 15.8 \\
\hline
\end{tabular}

a two-phase thermal unfolding (not shown for III*, but reported by Hagarman et al.). The respective visible $\mathrm{CD}$ signals suggest that all these thermally unfolded states are structurally distinct. For $\mathrm{III}_{u}, \mathrm{III}_{u}^{*}$, and $\mathrm{IV}_{u}$ the absorption and visible $C D$ spectra still suggest a low spin hexacoordinated state of the heme iron. The thermally unfolded state $V_{u}$ is somewhat mysterious. The rather large redshift of both the absorption and the Cotton band at high temperatures seems to be indicative of a ferrous pentacoordinated complex. 


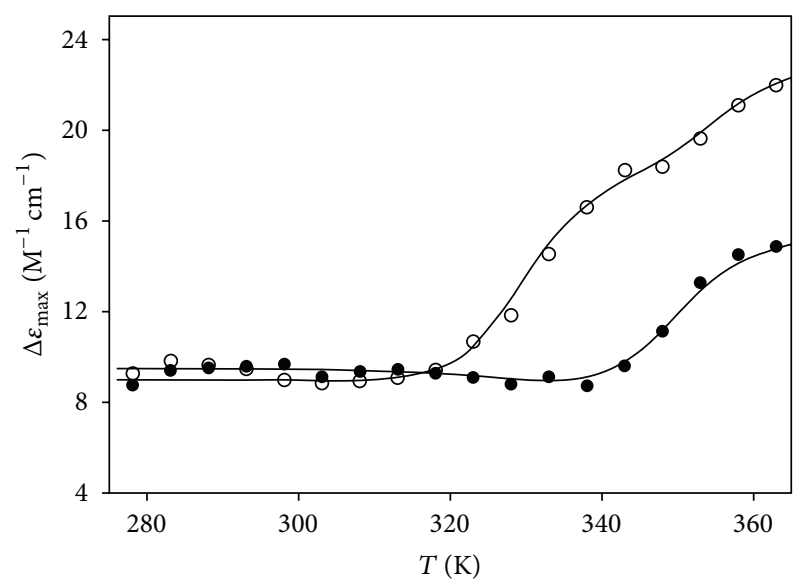

FIGURE 20: $\Delta \varepsilon$ versus temperature of oxidized bovine horse heart cytochrome c between 278 and $363 \mathrm{~K}$. The $\Delta \varepsilon$ values were obtained from the CD spectra in Figures 18 (state IV, filled circles) and 19 (state $\mathrm{V}$, open circles) at the position of the Cotton band maximum. The experimental data were taken from Hagarman et al. [88]. The solid lines result from fits described in the text and in [20], from where the figure was taken and modified.

If this is indeed the case, the thermal unfolding would be accompanied by a change of the protein's redox state.

Like many biochemical interactions, the folding/unfolding of proteins is governed by enthalpy-entropy compensation [133-137]. The compensation is exact at the folding temperature. The comparability of different folding processes can be judged based on the similarity of their folding/unfolding temperature. A closer look on the values listed in Table 2 reveals that, in particular, the values for $T_{u}$ are not very different indicating that very similar forces are involved in the respective unfolding processes. In this case, $\Delta H$ and $\Delta S$ show a high degree of correlation which can be described by [133]

$$
\Delta H=T_{c} \Delta S .
$$

If all folding temperatures of the considered processes are identical, $T_{c}$ equals these folding temperatures and the correlation coefficient for a fit of (11) to the experimental data will yield 1. A distribution of still similar transition temperatures leads to a representative $T_{c}$-value and correlation coefficients in the 0.97-0.99 regime [127].

It is interesting to compare the thermodynamic parameters listed in Table 2 with Gibbs energies of the HD exchange of cytochrome c unfolding reported by Krishna et al. (Table 3 ) [23]. The values of $\Delta G_{i}$ are by a factor of 3 lower than the $\Delta G_{\mathrm{HD}}$-values these authors reported for the infrared $\Omega$-loop, which is the least stable foldon, whereas the values obtained for $\Delta G_{u}$ are comparable with the $\Delta G_{\mathrm{HD}}$ values reported for the green helix/loop and the blue bihelix foldons. Remembering that the alkaline transition at room temperature involves the unfolding of the $\Omega$-foldons, we suspect that the $n \Rightarrow$ $i$ transition involves the destabilization of different foldons at different $\mathrm{pH}$, while the $i \Rightarrow u$ transition involves the higher lying foldons. The thermal unfolding reported by us in Hagarman et al. [88] and Schweitzer-Stenner et al. [20] is therefore somewhat different from the recently investigated thermal unfolding at low ionic strength [127]. Here we found that even the $i \Rightarrow u$ transition does not involve complete unfolding and leaves the blue foldon mostly intact over a rather large $\mathrm{pH}$ range from 1 to 12 . This shows that the presence of anions in solution does destabilize rather than stabilize cytochrome $\mathrm{c}$.

3.2. Oligomerization of Cytochrome c. We have mentioned above that a misfolded state of the protein is temporarily populated during cytochrome $\mathrm{c}$ folding at neutral $\mathrm{pH}$ and room temperature $[89,138-140]$. The nonnative states of the protein adopted at alkaline and acidic $\mathrm{pH}$ can be considered misfolded as well, because they bind the wrong ligand but show a high degree of folding otherwise. It is well documented that misfolded, as well as unfolded, proteins are prone to aggregation. Dobson even argued that the amyloidogenic state of proteins, in which the unfolded protein has formed rather longer fibrils of parallel or antiparallel $\beta$-sheets, is the most stable state for all proteins [141]. This would imply that the respective native states are in fact metastable, nonequilibrium states. With regard to cytochrome $\mathrm{c}$, thermal unfolding leads to protein aggregation into $\beta$-sheet structures and subsequent precipitation at temperatures above $350-360 \mathrm{~K}$ [142]. Acidic states I and II are more prone to aggregation than the alkaline state. State II can be converted into soluble aggregates even at moderate temperatures [139].

Besides aggregation into soluble and insoluble fibrils, cytochrome c exhibits another mode of aggregation which is much more interesting. It is well known for more than forty years that cytochrome $\mathrm{c}$ can form nonamyloidogenic oligomers after dissolving the protein in ethanol [143]. However, only recently Hirota et al. reported the crystal structure of the formed oligomers [144]. They found that dimers, trimers, and even tetramers of cytochrome $\mathrm{c}$ are formed by domain swapping; that is, the $\mathrm{C}$-terminal becomes displaced from its original position and links a protein with another one via nonbonding interactions (Figure 21). Here, monomers and oligomers are in equilibrium. The involved structural changes of the protein involve the dissociation of the $\mathrm{Fe}^{3+}$ M80 linkage and the ligation of the heme iron with $\mathrm{OH}^{-}$, which makes them reminiscent of state $\mathrm{V}_{b}$ as characterized by Döpner et al. [112]. This phenomenon of domain swapping was recently obtained for other proteins, such a serpin [145]. Subsequent investigations by Hayashi et al. revealed similar domain swapping for other cytochrome c species [29]. Most recently, they found that dimer formation via domain swapping occurs during the folding of the protein from a denatured state in solutions with moderate $\mathrm{GuHCl}$ concentrations [140]. Thus the formed cytochrome oligomers show enhanced peroxidase activities which made researchers wonder whether cytochrome $\mathrm{c}$ oligomers might be involved in such activities on the inner membrane of the mitochondria [146].

A rather peculiar strategy for obtaining cytochrome $\mathrm{c}$ oligomers with an intact secondary structure was recently reported by us [147]. We oxidized cytochrome $\mathrm{c}$ at $\mathrm{pH} 11.5$ (state $\mathrm{V}$ ) and allowed incubation times up to a week at this condition. After switching the solution back to more neutral $\mathrm{pH}$, the protein did not refold. We could show that 
TABLE 3: Thermodynamic parameters obtained from fitting the data in Figures 16, 18, and 20, reflecting the two-step thermal unfolding of the protein. Taken from [20].

\begin{tabular}{lcccc}
\hline & $\mathrm{pH} 7 \mathrm{MOPS}$ & $\mathrm{pH} \mathrm{7} \mathrm{Tris-HCl}$ & $\mathrm{pH} \mathrm{10.5}$ & $\mathrm{pH} \mathrm{11.5}$ \\
\hline$\Delta H_{i}^{0}[\mathrm{~kJ} / \mathrm{mol}]$ & 100 & 100 & 100 & 200 \\
$\Delta H_{u}^{0}[\mathrm{~kJ} / \mathrm{mol}]$ & 300 & 250 & 320 & 200 \\
$T_{i}[\mathrm{~K}]$ & 315 & 315 & 350 & 330 \\
$T_{u}[\mathrm{~K}]$ & 355 & 355 & -2.8 & 355 \\
$c_{p}[\mathrm{~kJ} / \mathrm{mol} \cdot \mathrm{K}]$ & -2.9 & -2.9 & -2.8 \\
\hline
\end{tabular}
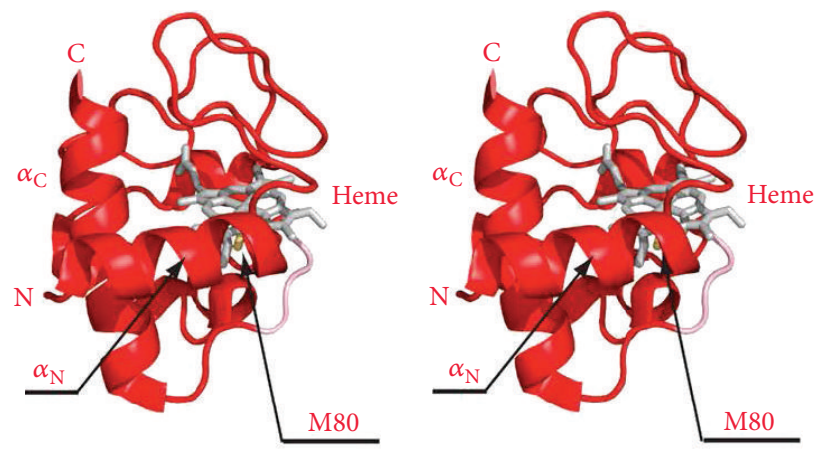

(a)
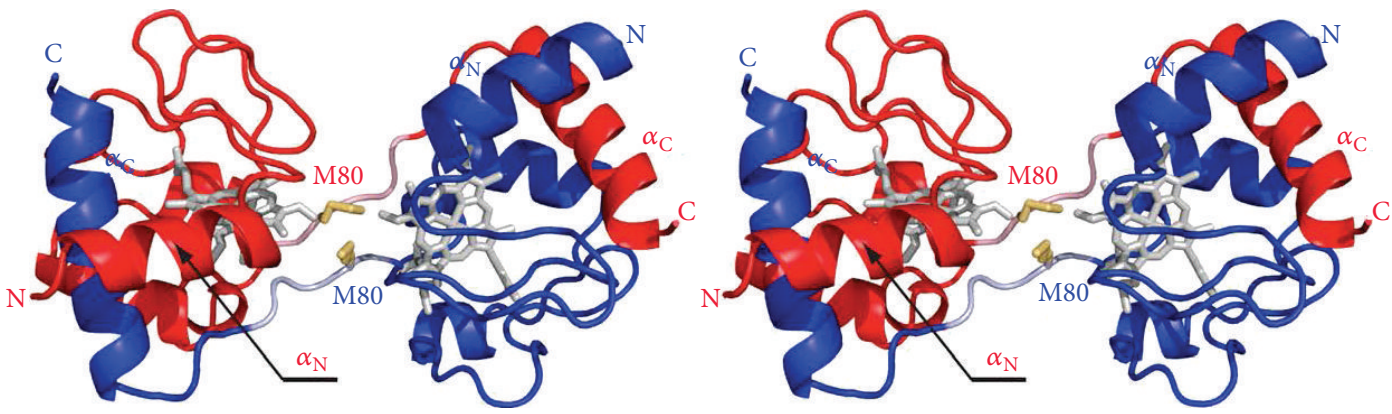

(b)
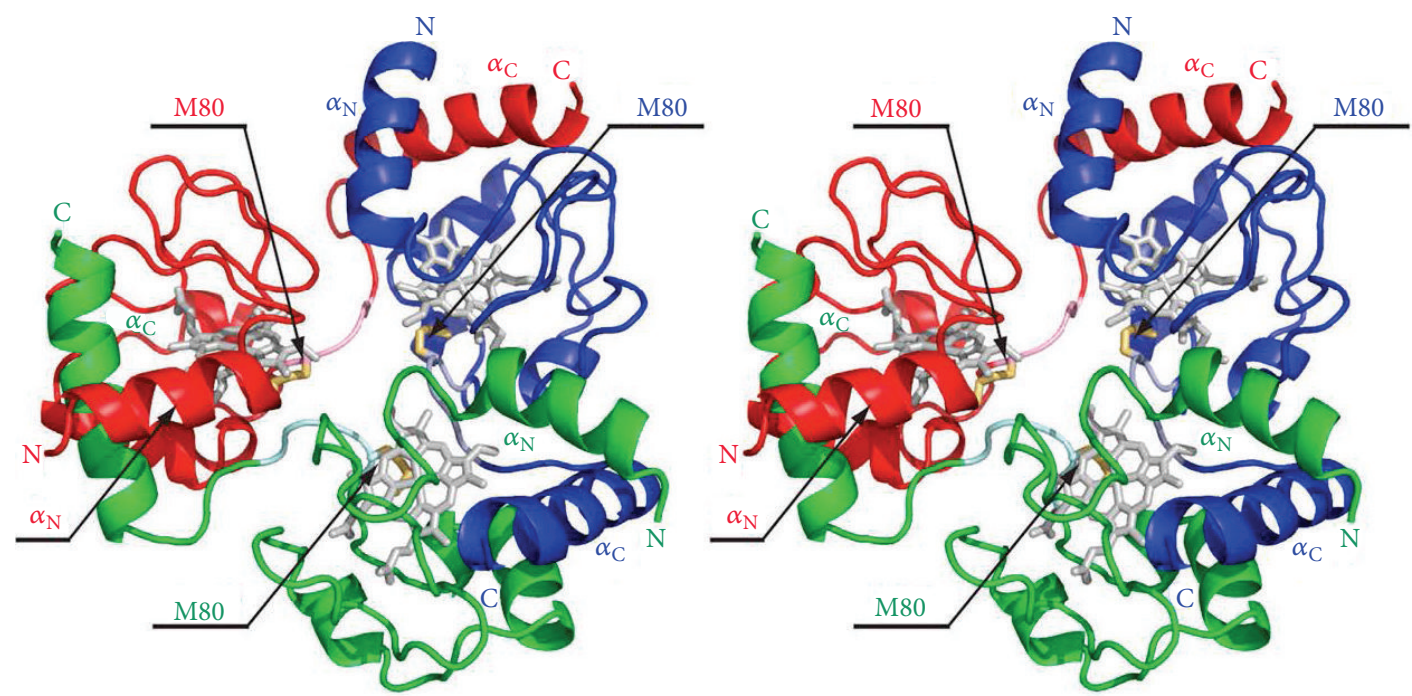

(c)

FIgURE 21: Crystal structures of oxidized monomeric and oligomeric cyt-c. (a) Structure of monomeric cyt-c (PDB ID code 1HRC). (b) Structure of dimeric cyt-c (red and blue). (c) Structure of trimeric cyt-c (red, blue, and green). The hemes are shown as gray stick models. Side-chain atoms of M80 are displayed as yellow stick models. The N and C termini and the N-and C-terminal helices are labeled as N, C, $\alpha_{\mathrm{N}}$, and $\alpha_{\mathrm{C}}$, respectively. The T79-A83 residues (hinge loop of the monomer) are depicted in pale colors. Taken from [144] with permission. 


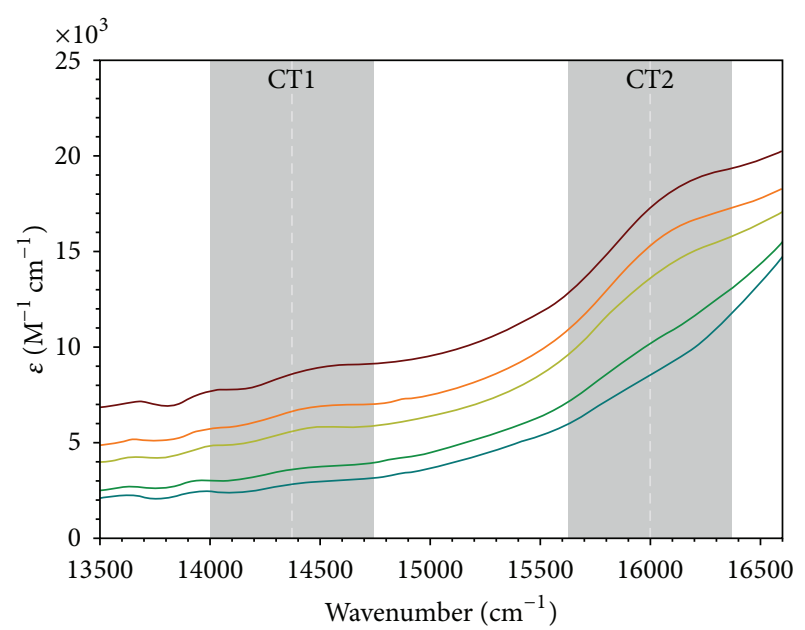

FIGURE 22: Charge transfer band region of the absorption spectrum of $0.5 \mathrm{mM}$ ferricytochrome $\mathrm{c}$ (equine) measured between 13,500 and $17,000 \mathrm{~cm}^{-1}$ in $0.1 \mathrm{mM}$ potassium phosphate buffer. Prior to the measurement the oxidized protein was exposed to alkaline conditions at $\mathrm{pH} 11.5$ for one week. The label CT1 exhibits the region of the so-called $695 \mathrm{~nm}$ charge transfer band, which is practically absent in the displayed spectra. CT2 is assignable to a heme $\rightarrow$ iron transition in high and quantum mixed heme irons. The spectrum was taken from [147] with permission.

this was not the result of chemical modifications. Moreover, we found that the protein predominantly stays monomeric at $50 \mu \mathrm{M}$ concentration, while substantial aggregation into dimers and trimers was obtained at $0.5 \mathrm{mM}$. Below $\mathrm{pH} 7$, a new nonnative species is formed which was probed by the occurrence of a weak absorption band at $625 \mathrm{~nm}$. Figure 22 shows the spectral region between $600 \mathrm{~nm}$ and $750 \mathrm{~nm}$ of the absorption spectrum of oxidized cytochrome $\mathrm{c}$ measured at the indicated $\mathrm{pH}$ after one-week oxidation at $\mathrm{pH} 11.5$. The $\mathrm{Fe}^{3+}-\mathrm{M} 80$ indicator at $695 \mathrm{~nm}$ is absent, indicating a nonnative state. The band at $625 \mathrm{~nm}$ appears below $\mathrm{pH} 6$ and gains intensity with decreasing $\mathrm{pH}$. A titration curve plotting the integrated intensity of the $625 \mathrm{~nm}$ band versus $\mathrm{pH}$ is plotted in Figure 23. An analysis of these data yielded apparent $\mathrm{pK}$-values of 4.7 and 6.4 .

The $625 \mathrm{~nm}$ band would normally be interpreted as indicative of a high spin hexa- or pentacoordinated states of the heme group [148-150]. However, resonance Raman data and the position of the respective absorption and $\mathrm{CD}$ bands in the Soret band region are inconsistent with such an interpretation. The only common denominator of all data sets is a quantum mixed state of the heme iron, as found in type C peroxidases such as horseradish peroxidase [151-154]. A quantum mixed state can be formed if the lowest iron state is high spin with an intermediate spin state not lying higher than $300 \mathrm{~cm}^{-1}$ [154]. In such a case the two states mix via spinorbit coupling. Such a quantum mixed state yields low spinlike Raman spectra and a charge transfer band in the region between 620 and $630 \mathrm{~nm}$, as obtained [155]. If the quantum mixed state is pentacoordinated, the Soret absorption peaks between respective low and high spin band positions, again in agreement with our absorption spectra [147].

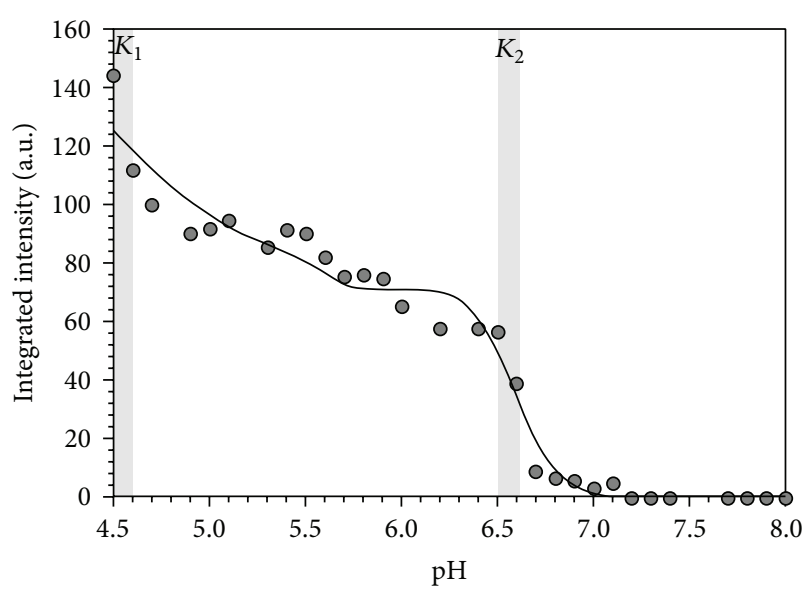

FIGURE 23: Integrated intensity of the CT2 band in the optical spectrum of horse heart ferricytochrome $\mathrm{c}$ incubated under oxidizing conditions at $\mathrm{pH} 11.5$ for seven days plotted as a function of $\mathrm{pH}$. The solid curve results from a fit explained in the text. The picture was taken from [147] and explained in the text.

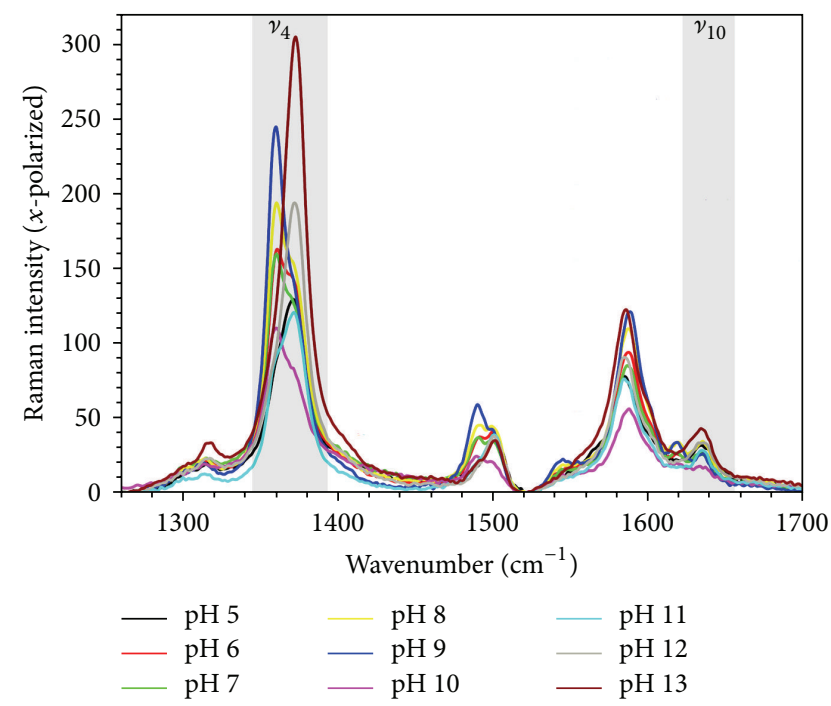

FIGURE 24: Resonance Raman spectra of $0.5 \mathrm{mM}$ ferricytochrome $\mathrm{c}$ measured at the indicated $\mathrm{pH}$ after the protein was subjected to oxidizing conditions at $\mathrm{pH} 11.5$ for 7 days. The spectra were taken with $442 \mathrm{~nm}$ near $B$-band excitation. The figure was taken from [147] with permission.

The UVCD spectra of this nonnative, apparently misfolded protein indicate rather intact secondary structure, which rules out amyloid formation. The oligomers obtained with $0.5 \mathrm{mM}$ concentration should therefore be assigned to domain swapping. Interestingly, these oligomers do not remain in the oxidized state. Resonance Raman (Figure 24) spectra clearly reveal coexisting oxidized and reduced states, the ratio being $\mathrm{pH}$ dependent. The population of the reduced state becomes maximal between $\mathrm{pH} 8$ and 9 and decreases substantially towards acidic and alkaline $\mathrm{pH}$. This is indicative of a yet undiscovered proton-redox linkage, which is currently under investigation. 
The work described above reveals that a pronounced frustration occurs along the alkaline pathway provided that the protein is allowed to stay longer at $\mathrm{pH} 11.5$ in state $\mathrm{V}$. A less effective frustration was earlier reported for acid unfolding [156]. Frustration means that the protein occupies an intermediate state during the folding process which is separated by a large barrier from the native state. Since resonance Raman data obtained with $0.5 \mathrm{mM}$ protein concentration exhibit spin and oxidation marker lines close to positions found in $\mathrm{OH}^{-}$ ligated hemes, Soffer et al. conjectured that the mixture of $\mathrm{V}_{a}$ and $\mathrm{V}_{b}$ changes over time in that the predominant fraction of $\mathrm{V}_{a}$ converts into $\mathrm{V}_{b}$ over time. If this is true it becomes understandable that state $\mathrm{V}$ refolds without any problems if the protein stays at the corresponding alkaline $\mathrm{pH}$ only for a short period of time. On the contrary, refolding of $\mathrm{V}_{b}$ might be much more frustrating, that is, misfolded; nonnative states become occupied very early on in its downhill motion in the folding funnel. Since the respective UVCD spectra indicate a lot of helical content, it can be assumed that at least the $\mathrm{N}$ - and C-helices are fully folded. Since they do not form the native contacts, the former is free for domain swapping, thus producing dimers, trimers, and even tetramers with a rather regular and ordered quaternary structure. This is likely to stabilize the misfolded state. Zheng et al. used the funneltheory to investigate the formation of multidomain aggregates during the folding process [157]. Their results indeed suggest that such oligomers might be more stable than the native state and that protein are prone to misfolding at mildly unfolding conditions. That is exactly what we obtained [147].

\section{Unfolding of Cytochrome c on Surfaces}

All folding studies discussed in the preceding paragraph dealt with cytochrome $c$ in solution. While of general interest for the protein folding community in general and the heme protein community in particular, the obtained results might be considered of limited relevance for an understanding of the protein's function owing to the fact that its natural environment is the inner membrane of the mitochondria, to which a fraction of the cytochromes in the intermembrane space is bound [158]. Investigating the protein in vivo and in vitro is rather challenging, even though various attempts have been made $[158,159]$. Over the last ten years two types of surfaces have emerged as suitable model system to study structure and function of cytochrome $c$ in a more realistic environment: functionalized self-assembled monolayers and anionic phospholipid liposomes. In what follows, experiments with these two types of surfaces are briefly discussed.

4.1. Unfolding of Cytochrome c on Electrode Surfaces. Murdiga, Hildebrandt, and their respective coworkers used spectroscopic and electrochemical techniques to study cytochrome immobilized on gold and silver electrodes coated with self-assembled monolayers of, for example, pyridine terminated alkanethiols $[160,161]$. They found a potential dependent equilibrium between two coordination states, namely, a pentacoordinated high spin and a hexacoordinated low spin state of the heme iron. In the latter the M80 ligand was found to be replaced by a pyridinyl residue, which obviously requires that the respective structures are different from the native one. The two states were found to differ with respect to their redox potentials. In another approach, Wackerbarth and Hildebrandt coated electrodes with anions of appropriate acids which produced an array of negative charges on metal surfaces [162]. This way they mimicked to some extent the anionic surface of the mitochondrial inner membrane. Cytochrome $c$ can easily bind to such a surface via the positive patches on its surface. The authors observed a potential dependent equilibrium between two states termed $B 1$ and $B 2$. The former is the authors' notation for the native state III, and the latter is partially unfolded and involves a variety of hexacoordinated and pentacoordinated subspecies, with the former exhibiting bis-histidine complexes. Correspondingly their redox potential was found to vary between -0.425 and $-0.385 \mathrm{~V}$.

More recently, Monari et al. used surface enhanced resonance Raman spectroscopy and electrochemical measurements to investigate the influence of urea on surface immobilized yeast cytochrome $\mathrm{c}$ and its K72AK73AK79A [163]. They found that, above a urea concentration of ca. $6 \mathrm{M}$, the native M80 ligand is replaced by a histidine ligand, reminiscent of what one finds in solution. This structural change was found to cause a $0.4 \mathrm{~V}$ shift of the redox potential towards negative values. They found further that the transition to the nonnative state requires slightly less urea for the triple mutant, which they interpreted as indicating a role of the three replaced lysines with regard to the stabilization of the native structure on the surface. Interestingly, these authors found a nearly perfect enthalpy-entropy compensation with regard to the Gibbs energy contribution to the redox potential, which the authors measured for different urea concentrations. In a subsequent study Ranieri et al. showed that yeast cytochrome immobilized on the type of electrode used by Monari et al. became what the authors termed a pseudoperoxidase in the presence of urea [164]. The protein's activity involved a two-electron reduction of water. The Michaelis-Menten-like parameters used to describe the process were shown to be affected by the above replacements of the lysine residues by alanine.

4.2. Unfolding of Cytochrome c on Anionic Liposome Surfaces. Apoptosis is essential for maintaining the functioning of the human body. Damaged, genetically modified, or otherwise unwanted cells are eliminated by a regulated chain of biochemical reactions. In an average human adult, apoptosis causes the death of between 50 and 70 billion cells each day [16]. While apoptosis is generally beneficial, defective apoptotic processes can lead to an extensive variety of diseases. Excessive apoptosis causes atrophy, whereas an insufficient amount results in uncontrolled cell proliferation, such as cancer. Therefore, gaining a complete and thorough understanding of the parameters governing the apoptosis pathway is of great importance for any therapeutic approach aimed at battling such defective behavior.

In the inner membrane space of mitochondria, cytochrome $c$ exhibits a delicate balance between membrane bound and free cytochromes. Cortese et al., by performing 
a conformational analysis of cytochrome $\mathrm{c}$ in mitochondria, reported that the protein can adopt nonnative states when bound to the IMM [158]. A characteristic of the IMM is its high cardiolipin (CL, Figure 25) content; the biphosphate head group is the main target for cytochrome $\mathrm{c}$ binding. Studies carried out over the last 15 years have revealed a rather complex and yet incomplete picture of cytochrome $\mathrm{c}$ binding to CL-containing membranes, which was mostly obtained by using liposomes as model systems. The most important results are briefly reviewed below.

The very comprehensive work of Rytömaa and Kinnunen indicates the existence of two protein binding sites of horse heart cytochrome $c$ termed A and C [165]. Site A binding is purely electrostatic and can therefore be inhibited by salt $(\mathrm{NaCl})$. It is formed by the positively charged residues (mostly $\mathrm{K})$ on the protein's surface. Site C involves hydrogen bonding and requires a slightly acidic $\mathrm{pH}$ and might also involve the penetration of a lipid chain into the interior of the protein (extended lipid anchorage). Kalanxhi and Wallace provided support for the notion that another mode of lipid anchorage is actually associated with the A-site binding [166]. The latter notion is supported by Rytömaa and Kinnunen, who found that while the presence of salt could prevent A-site binding, bound cytochrome did not dissociate from the membrane $[165,167]$. Bregstrom et al., by investigating the binding for ferricytochrome $\mathrm{c}$ to single-giant unilamellar vesicles, found strong evidence for pore formation by cytochrome c-CL complexes [168]. The picture of cytochrome-liposome interactions became even more complex recently due to work of Kawai et al., who provided evidence for a third binding site $\mathrm{L}$ nearly opposite to $\mathrm{A}$, which as site $\mathrm{C}$ is more effective at acidic $\mathrm{pH}$ [169].

Pletneva and coworkers have recently applied their FRET technique to study ferricytochrome $\mathrm{c}$ on liposomes with different CL contents [170-173]. As observed for unfolded or partially unfolded cytochrome in solution, they identified an equilibrium between compact and extended states. The fraction of the latter was found to increase with CL content, until it saturated at a fraction of 0.5 [172]. The authors also investigated the influence of $\mathrm{NaCl}$ and found the extended state to be depleted at high ionic strength. Interestingly, they found no indication for the $\mathrm{C}$-sites and the lipid anchorage reported by Rytömaa and Kinnunen [165]. Sinibaldi et al. used visible circular dichroism to study the binding of ferricytochrome to 100\% CL liposomes [174]. They identified two binding sites with rather different binding affinities. Both respond to the addition of $\mathrm{NaCl}$, which seems to suggest that the binding is electrostatic in nature. In this case, neither one of these binding sites can be associated with site $\mathrm{C}$ proposed by Kinnunen and coworkers, since it would not be susceptible to $\mathrm{Na}^{+}$induced inhibition.

The picture which emerges from all these different binding and protein conformation studies is far from being consistent. A couple of issues deserve to be mentioned. First, none of the above studies probed the state of the unbound protein; hence it was not checked whether the conformational transition of the protein is really reversible. Very recently, Pandiscia and Schweitzer-Stenner found that this is not the case in the absence of salt [175]. Instead a major fraction of

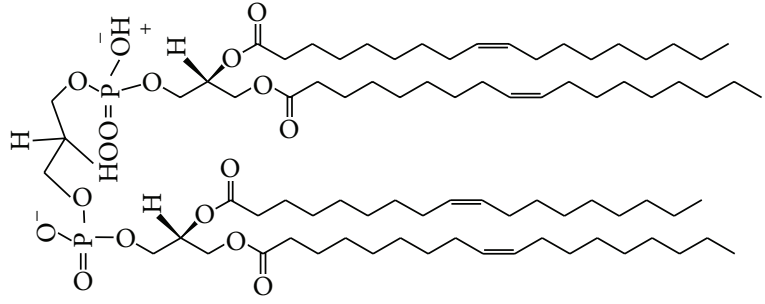

FIGURE 25: Schematic representations of cardiolipin. The figure has been taken from open sources.

the protein stays in a semiunfolded state, exhibiting substantial tryptophan fluorescence. However, the addition of salt not only dissociates the protein, but also converts most of the protein to the native or a native-like state. These results show that cytochrome $\mathrm{c}$ binding to anionic liposomes cannot be described in terms of rather simple equilibrium laws. Second, it is noteworthy that the concentration ranges of the experiments conducted by the different groups are rather different. Rytömaa and coworkers observed their Astate binding by titrating cytochrome $\mathrm{c}$ in the submicromolar range to an access of lipids $[165,167]$. Their binding reaches saturation at a cytochrome $\mathrm{c}$ concentration well below the total accessible CL content of the mixture $(\sim 0.9 \mu \mathrm{M})$. The binding studies performed by the Pletneva, Santucci, and Schweitzer-Stenner groups were carried out by adding an increasing number of lipids (i.e., liposomes) to a larger amount of cytochrome c $(3 \mu \mathrm{M}$ was reported by Hanske et al. [173], $10 \mu \mathrm{M}$ was used by Sinibaldi et al. [174], and $5 \mu \mathrm{M}$ was employed in our study (Pandiscia and SchweitzerStenner, [159]). The binding affinities reflected by their isotherms are much lower than that for Kinnunen's A-site. Further experiments, which involve an analysis of unbound proteins, are apparently necessary for the development of a comprehensive binding model. These are currently on their way in our laboratory.

\section{Summary and Outlook}

Cytochrome $\mathrm{c}$ in its oxidized state is a very flexible molecule, contrary to its reduced counterpart. The reason for this enormous difference in stability is the reduced strength of the Fe-M80 linkage in the oxidized state. It requires less energy to break and thus the pK-values of acid and alkaline transitions, as well as the thermal transition temperature, are much lower than in the reduced state. Ferricytochrome $c$ adopts a variety of different states at acidic and alkaline $\mathrm{pH}$ and at high temperatures with tertiary structures which deviate from the native one, while most of the secondary structure remains rather intact. With the exception of state $\mathrm{I}$, which we did not discuss in detail, the $\mathrm{H} 18-\mathrm{Fe}^{3+}$ linkage is maintained. The covalent linkage of the heme to the CAQC region is always intact, which prevents a segment of the protein from unfolding. We found a nonnative state which appears stable at neutral $\mathrm{pH}$ and room temperature after the protein was incubated at alkaline $\mathrm{pH}$ for a week [147]. We interpreted this as the population of a frustrated 
metastable state in the protein's folding funnel, but one might wonder whether this state is even energetically lower than the native state which is just separated from it by a large enough barrier to avoid spontaneous unfolding in the oxidized state. We are planning a systematic investigation of the determinants of the processes underlying the formation of this misfiled state and its propensity for aggregation of nonamyloidogenic aggregates via domain swapping. The $\mathrm{pH}-$ window from which the formation of this state becomes possible has still to be identified. Currently, we are doing some long-term experiments to probe the kinetics of the protein's recovery from misfolding. Preliminary data indicate that this might take longer than a year. The results of these investigations will substantially enrich our understanding of protein folding. They will prompt searches for conditions at which other proteins show a similar behavior.

Cytochrome $\mathrm{c}$ has now been researched for over 70 years. One might think that this is enough and that researchers should turn to newer pastures. This view is belied by the realities. The results of Soffer et al. show that the energy landscape of the protein is yet not fully understood. Exploring it further is likely to reveal important general insights about the relationship between folded and misfolded states of proteins. We wonder whether cytochrome c oligomers formed by domain swapping might have exciting switchable redox properties. With regard to the protein's function in mitochondria it still needs to be explored how the environmental conditions maintain a balance between intact (necessary for electron transfer) and partially unfolded proteins (necessary for gaining peroxidase activity and for other functions of the protein not discussed in this review). Studying this relationship should transcend the realm of cytochrome $\mathrm{c}$ by shedding light on how membrane surfaces can unfold proteins, thus tuning them to another function. In this context the role of the strong electric field at the membrane surface needs to be explored further. Theoretical and computational studies have revealed that strong electric fields can facilitate the unfolding of ferricytochrome c. Needless to say that any results regarding this issue would be of most general relevance for understanding protein structure on membranes.

\section{Conflict of Interests}

The author declares that there is no conflict of interests regarding the publication of this paper.

\section{Acknowledgments}

Coworkers and collaborators have over time contributed to the research of the group, which is discussed in this review. During the author's time at the University of Bremen these were Dr. Ulrich Bobinger and Professor Wolfgang Dreybrodt. At the author's current institution, Drexel University, Dr. Qing Huang, Dr. Andrew Hagarman, Isabelle Dragomir, Ronak Shah, Daniel Verbaro, Emma Fradkin, Dr. Jonathan B. Soffer, and Leah Pandiscia contributed to the reported research. The author enjoyed collaborations with Professor Carmichael Wallace from Dalhousie University in Halifax,
Canada, with Professor Antonio Cupane and Dr. Matteo Levantino from the University of Palermo in Italy and with the late Dr. Monique Laberge from the St-Jean Military College in Richelain, Canada. The author thanks Leah Pandiscia for the critical reading of the paper.

\section{References}

[1] M. Paoli, J. Marles-Wright, and A. Smith, "Structure-function relationships in heme-proteins," DNA and Cell Biology, vol. 21, no. 4, pp. 271-280, 2002.

[2] E. Antonini and M. Brunori, Hemoglobin and Myoglobin in Their Reaction with Ligands, Elsevier, Amsterdam, The Netherlands, 1970.

[3] C. Weber, B. Michel, and H. R. Bosshard, "Spectroscopic analysis of the cytochrome $\mathrm{c}$ oxidase-cytochrome c complex: circular dichroism and magnetic circular dichroism measurements reveal change of cytochrome c heme geometry imposed by complex formation.", Proceedings of the National Academy of Sciences of the United States of America, vol. 84, no. 19, pp. 66876691, 1987.

[4] E. T. Adman, "Comparison of the structures of electron transfer proteins," Biochimica et Biophysica Acta, vol. 549, no. 2, pp. 107$144,1979$.

[5] N. C. Veitch, "Horseradish peroxidase: a modern view of a classic enzyme," Phytochemistry, vol. 65, no. 3, pp. 249-259, 2004.

[6] M. J. Coon, “Cytochrome P450: nature's most versatile biological catalyst," Annual Review of Pharmacology and Toxicology, vol. 45, pp. 1-25, 2005.

[7] E. R. Derbyshire and M. A. Marletta, "Structure and regulation of soluble guanylate cyclase," Annual Review of Biochemistry, vol. 81, pp. 533-559, 2012.

[8] H. N. Kirkman and G. F. Gaetani, "Catalase: a tetrameric enzyme with four tightly bound molecules of NADPH," Proceedings of the National Academy of Sciences of the United States of America, vol. 81, no. 14 I, pp. 4343-4347, 1984.

[9] A. M. Amoia and W. R. Montfort, "Apo-nitrophorin 4 at atomic resolution," Protein Science, vol. 16, no. 9, pp. 2076-2081, 2007.

[10] F. A. Walker, "Magnetic spectroscopic (EPR, ESEEM, Mössbauer, MCD and NMR) studies of low-spin ferriheme centers and their corresponding heme proteins," Coordination Chemistry Reviews, vol. 185-186, pp. 471-534, 1999.

[11] R. Schweitzer-Stenner, "Allosteric linkage-induced distortions of the prosthetic group in haem proteins as derived by the theoretical interpretation of the depolarization ratio in resonance Raman scattering," Quarterly Reviews of Biophysics, vol. 22, no. 4, pp. 381-479, 1989.

[12] N. A. Belikova, Y. A. Vladimirov, A. N. Osipov et al., "Peroxidase activity and structural transitions of cytochrome $\mathrm{C}$ bound to cardiolipin-containing membranes," Biochemistry, vol. 45, no. 15, pp. 4998-5009, 2006.

[13] G. G. Borisenko, A. A. Kapralov, V. A. Tyurin, A. Maeda, D. A. Stoyanovsky, and V. E. Kagan, "Molecular design of new inhibitors of peroxidase activity of cytochrome c/cardiolipin complexes: fluorescent oxadiazole-derivatized cardiolipin," Biochemistry, vol. 47, no. 51, pp. 13699-13710, 2008.

[14] V. E. Kagan, V. A. Tyurin, J. Jiang et al., "Cytochrome c acts as a cardiolipin oxygenase required for release of proapoptotic factors," Nature Chemical Biology, vol. 1, no. 4, pp. 223-232, 2005. 
[15] A. A. Kapralov, I. V. Kurnikov, I. I. Vlasova et al., "The hierarchy of structural transitions induced in cytochrome $\mathrm{c}$ by anionic phospholipids determines its peroxidase activation and selective peroxidation during apoptosis in cells," Biochemistry, vol. 46, no. 49, pp. 14232-14244, 2007.

[16] X. Jiang and X. Wang, "Cytochrome C-mediated apoptosis," Annual Review of Biochemistry, vol. 73, pp. 87-106, 2004.

[17] M. J. Burkitt and P. Wardman, "Cytochrome $c$ is a potent catalyst of dichlorofluorescin oxidation: Implications for the role of reactive oxygen species in apoptosis," Biochemical and Biophysical Research Communications, vol. 282, no. 1, pp. 329333, 2001.

[18] S. W. Englander, T. R. Sosnick, L. C. Mayne, M. Shtilerman, P. X. Qi, and Y. Bai, "Fast and slow folding in cytochrome $c$," Accounts of Chemical Research, vol. 31, no. 11, pp. 737-744, 1998.

[19] R. Schweitzer-Stenner, "Polarized resonance Raman dispersion spectroscopy on metalporphyrins," Journal of Porphyrins and Phthalocyanines, vol. 5, no. 3, pp. 198-224, 2001.

[20] R. Schweitzer-Stenner, A. Hagarman, D. Verbaro, and J. B. Soffer, "Conformational stability of cytochrome C probed by optical spectroscopy.", Methods in enzymology, vol. 466, pp. 109-153, 2009.

[21] E. K. J. Tuominen, C. J. A. Wallace, and P. K. J. Kinnunen, "Phospholipid-cytochrome $c$ interaction," The Journal of Biological Chemistry, vol. 277, pp. 8822-8826, 2002.

[22] W. Humphrey, A. Dalke, and K. Schulten, "VMD: visual molecular dynamics," Journal of Molecular Graphics, vol. 14, no. 1, pp. 33-38, 1996.

[23] M. M. G. Krishna, H. Maity, J. N. Rumbley, Y. Lin, and S. W. Englander, "Order of steps in the cytochrome c folding pathway: evidence for a sequential stabilization mechanism," Journal of Molecular Biology, vol. 359, no. 5, pp. 1410-1419, 2006.

[24] J. B. Soffer, The Folded, Partially Folded, Misfolded, and Unfolded Conformations of Cytochrome c Probed by Optical Spectroscopy, Department of Chemistry, Drexel University, Philadelphia, Pa, USA, 2013.

[25] G. V. Louie and G. D. Brayer, "High-resolution refinement of yeast iso-1-cytochrome $\mathrm{c}$ and comparisons with other eukaryotic cytochromes c," Journal of Molecular Biology, vol. 214, no. 2, pp. 527-555, 1990.

[26] G. W. Bushnell, G. V. Louie, and G. D. Brayer, "High-resolution three-dimensional structure of horse heart cytochrome $c$," Journal of Molecular Biology, vol. 214, no. 2, pp. 585-595, 1990.

[27] P. Weinkam, E. V. Pletneva, H. B. Gray, J. R. Winkler, and P. G. Wolynes, "Electrostatic effects on funneled landscapes and structural diversity in denatured protein ensembles," Proceedings of the National Academy of Sciences of the United States of America, vol. 106, no. 6, pp. 1796-1801, 2009.

[28] G. J. Pielak, D. S. Auld, J. R. Beasley et al., "Protein thermal denaturation, side-chain models, and evolution: amino acid substitutions at a conserved helix-helix interface," Biochemistry, vol. 34, no. 10, pp. 3268-3276, 1995.

[29] Y. Hayashi, S. Nagao, H. Osuka, H. Komori, Y. Higuchi, and S. Hirota, "Domain swapping of the heme and N-terminal $\alpha$-helix in Hydrogenobacter thermophilus cytochrome $c_{552}$ dimer," Biochemistry, vol. 51, no. 43, pp. 8608-8616, 2012.

[30] G. Zoppellaro, E. Harbitz, R. Kaur, A. A. Ensign, K. L. Bren, and K. K. Andersson, "Modulation of the ligand-field anisotropy in a series of ferric low-spin cytochrome $c$ mutants derived from Pseudomonas aeruginosa cytochrome $c$-551 and Nitrosomonas europaea cytochrome c-552: A Nuclear Magnetic Resonance and Electron Paramagnetic Resonance study," Journal of the American Chemical Society, vol. 130, no. 46, pp. 15348-15360, 2008.

[31] J. M. Friedman, "Structure, dynamics, and reactivity in hemoglobin," Science, vol. 228, no. 4705, pp. 1273-1280, 1985.

[32] W. Jentzen, J. Ma, and J. A. Shelnutt, "Conservation of the conformation of the porphyrin macrocycle in hemoproteins," Biophysical Journal, vol. 74, no. 2, pp. 753-763, 1998.

[33] I. Rasnik, K. A. Sharp, J. A. Fee, and J. M. Vanderkooi, "Spectral analysis of cytochrome c: effect of heme conformation, axial ligand, peripheral substituents, and local electric fields," Journal of Physical Chemistry B, vol. 105, no. 1, pp. 282-286, 2001.

[34] N. V. Prabhu, S. D. Dalosto, K. A. Sharp, W. W. Wright, and J. M. Vanderkooi, "Optical spectra of Fe(II) cytochrome c interpreted using molecular dynamics simulations and quantum mechanical calculations," Journal of Physical Chemistry B, vol. 106, no. 21, pp. 5561-5571, 2002.

[35] E. S. Manas, W. W. Wright, K. A. Sharp, J. Friedrich, and J. M. Vanderkooi, "The influence of protein environment on the low temperature electronic spectroscopy of Zn-substituted cytochrome," Journal of Physical Chemistry B, vol. 104, no. 29, pp. 6932-6941, 2000.

[36] M. Laberge, M. Köhler, J. M. Vanderkooi, and J. Friedrich, "Sampling field heterogeneity at the heme of c-type cytochromes by spectral hole burning spectroscopy and electrostatic calculations," Biophysical Journal, vol. 77, no. 6, pp. 3293-3304, 1999.

[37] R. Schweitzer-Stenner, "Internal electric field in cytochrome C explored by visible electronic circular dichroism spectroscopy," Journal of Physical Chemistry B, vol. 112, no. 33, pp. 10358-10366, 2008.

[38] A. K. Churg and A. Warshel, "Control of the redox potential of cytochrome $c$ and microscopic dielectric effects in proteins," Biochemistry, vol. 25, no. 7, pp. 1675-1681, 1986.

[39] A. M. Berghuis, J. G. Guillemette, M. Smith, and G. D. Brayer, "Mutation of tyrosine-67 to phenylalanine in cytochrome $c$ significantly alters the local heme environment," Journal of Molecular Biology, vol. 235, no. 4, pp. 1326-1341, 1994.

[40] C. Blouin and C. J. A. Wallace, "Protein matrix and dielectric effect in cytochrome $c$," The Journal of Biological Chemistry, vol. 276, no. 31, pp. 28814-28818, 2001.

[41] R. Schweitzer-Stenner, Q. Huang, A. Hagarman, M. Laberge, and C. J. A. Wallace, "Static normal coordinate deformations of the heme group in mutants of ferrocytochrome $c$ from Saccharomyces cerevisiae probed by resonance Raman spectroscopy," Journal of Physical Chemistry B, vol. 111, no. 23, pp. 6527-6533, 2007.

[42] B. R. Gelin, A. W. Lee, and M. Karplus, "Hemoglobin tertiary structural change on ligand binding its role in the co-operative mechanism," Journal of Molecular Biology, vol. 171, no. 4, pp. 489-559, 1983.

[43] T. G. Spiro, "The resonance Raman spectra of metalloporphyrins and heme proteins," in Iron Porphyrins, A. B. P. Lever and H. B. Gray, Eds., pp. 89-166, Addison-Wesley, London, UK, 1993.

[44] T. G. Spiro and T. C. Strekas, "Resonance Raman spectra of heme proteins. Effects of oxidation and spin state," Journal of the American Chemical Society, vol. 96, no. 2, pp. 338-345, 1974.

[45] S. Hu, K. M. Smith, and T. G. Spiro, "Assignment of protoheme Resonance Raman spectrum by heme labeling in myoglobin," Journal of the American Chemical Society, vol. 118, no. 50, pp. 12638-12646, 1996. 
[46] S. Hu, I. K. Morris, J. P. Singh, K. M. Smith, and T. G. Spiro, "Complete assignment of cytochrome c resonance Raman spectra via enzymatic reconstitution with isotopically labeled hemes," Journal of the American Chemical Society, vol. 115, no. 26, pp. 12446-12458, 1993.

[47] S. Choi, T. G. Spiro, K. C. Langry, K. M. Smith, D. L. Budd, and G. N. La Mar, "Structural correlations and vinyl influences in resonance raman spectra of protoheme complexes and proteins," Journal of the American Chemical Society, vol. 104, no. 16, pp. 4345-4351, 1982.

[48] B. R. Stallard, P. R. Callis, P. M. Champion, and A. C. Albrecht, "Application of the transform theory to resonance Raman excitation profiles in the Soret region of cytochrome-c," The Journal of Chemical Physics, vol. 80, no. 1, pp. 70-82, 1984.

[49] P. M. Champion and A. C. Albrecht, "Investigations of Soret excited resonance Raman excitation profiles in cytochrome c," The Journal of Chemical Physics, vol. 71, no. 3, pp. 1110-1121, 1979.

[50] D. L. Brautigan, B. A. Feinberg, B. M. Hoffman, E. Margoliash, J. Preisach, and W. E. Blumberg, "Multiple low spin forms of the cytochrome $\mathrm{c}$ ferrihemochrome. EPR spectra of various eukaryotic and prokaryotic cytochromes c," Journal of Biological Chemistry, vol. 252, no. 2, pp. 574-582, 1977.

[51] G. N. LaMar and J. S. Satterlee, "Nuclear magnetic resonance of heme proteins," in The Porphyrin Handbook, K. M. Kadish, K. M. Smith, and R. Guilard, Eds., Academic Press, San Francisco, Cailf, USA, 2000.

[52] G. N. la Mar, D. L. Budd, D. B. Viscio, K. M. Smith, and K. C. Langry, "Proton nuclear magnetic resonance characterization of heme disorder in hemoproteins," Proceedings of the National Academy of Sciences of the United States of America, vol. 75, no. 12, pp. 5755-5759, 1978.

[53] H. Senn, M. Billeter, and K. Wüthrich, "The spatial structure of the axially bound methionine in solution conformations of horse ferrocytochrome $\mathrm{c}$ and Pseudomonas aeruginosa ferrocytochrome c 551 by ${ }^{1} \mathrm{H}$ NMR," European Biophysics Journal, vol. 11, no. 1, pp. 3-15, 1984.

[54] D. W. Collins, D. D. Fitchen, and A. Lewis, "Resonance Raman scattering from cytochrome $c$. The frequency dependence of the depolarization ratio," Journal of Chemical Physics, vol. 59, no. 10, pp. 5714-5719, 1973.

[55] J. A. Shelnutt, "The Raman excitation spectra and absorption spectrum of a metalloporphyrin in an environment of low symmetry," The Journal of Chemical Physics, vol. 72, no. 7, pp. 3948-3958, 1980.

[56] M. Z. Zgierski and M. Pawlikowski, "Depolarization dispersion curves of resonance Raman fundamentals of metalloporphyrins and metallophthalocyanines subject to asymmetric perturbations," Chemical Physics, vol. 65, no. 3, pp. 335-367, 1982.

[57] R. Schweitzer-Stenner and W. Dreybrodt, "Excitation profiles and depolarization ratios of some prominent Raman lines in oxyhemoglobin and ferrocytochrome $\mathrm{C}$ in the pre-resonant and resonant region of the Q-band," Journal of Raman Spectroscopy, vol. 16, pp. 111-123, 1985.

[58] R. Schweitzer-Stenner and W. Dreybrodt, "Investigation of Haem-Protein coupling and structural heterogeneity in myoglobin and haemoglobin probed by resonance raman spectroscopy," Journal of Raman Spectroscopy, vol. 23, pp. 539-550, 1991.

[59] R. Schweitzer-Stenner, A. Cupane, M. Leone, C. Lemke, J. Schott, and W. Dreybrodt, "Anharmonic protein motions and heme deformations in myoglobin cyanide probed by absorption and Resonance Raman spectroscopy," Journal of Physical Chemistry B, vol. 104, no. 19, pp. 4754-4764, 2000.

[60] R. Schweitzer-Stenner, U. Bobinger, and W. Dreybrodt, "Multimode analysis of depolarization ratio dispersion and excitation profiles of seven Raman fundamentals from the haeme group in ferrocytochrome c," Journal of Raman Spectroscopy, vol. 22, no. 2, pp. 65-78, 1991.

[61] M. Levantino, Q. Huang, A. Cupane, M. Laberge, A. Hagarman, and R. Schweitzer-Stenner, "The importance of vibronic perturbations in ferrocytochrome c spectra: A reevaluation of spectral properties based on low-temperature optical absorption, resonance Raman, and molecular-dynamics simulations," Journal of Chemical Physics, vol. 123, no. 5, Article ID 054508, 2005.

[62] E. Unger, U. Bobinger, W. Dreybrodt, and R. SchweitzerStenner, "Vibronic coupling in $\mathrm{Ni}(\mathrm{II})$ porphine derived from resonant Raman excitation profiles," Journal of Physical Chemistry, vol. 97, no. 39, pp. 9956-9968, 1993.

[63] R. Schweitzer-Stenner, A. Stichternath, W. Dreybrodt et al., "Raman dispersion spectroscopy on the highly saddled nickel(II)-octaethyltetraphenylporphyrin reveals the symmetry of nonplanar distortions and the vibronic coupling strength of normal modes," Journal of Chemical Physics, vol. 107, no. 6, pp. 1794-1815, 1997.

[64] R. Schweitzer-Stenner and J. B. Soffer, "Optical spectroscopy," in Biophysical Techniques for Structural Characterization of Macromolecules, H. J. Dyson, Ed., pp. 533-591, Oxford Academic Press, Oxford, UK, 2012.

[65] X. Li, R. S. Czernuszewicz, J. R. Kincaid, P. Stein, and T. G. Spiro, "Consistent porphyrin force field-2. Nickel octaethylporphyrin skeletal and substituent mode assignments from ${ }^{15} \mathrm{~N}$, meso- $\mathrm{d}_{4}$, and methylene- $\mathrm{d}_{16}$ Raman and infrared isotope shifts," Journal of Physical Chemistry, vol. 94, no. 1, pp. 47-61, 1990.

[66] C. Lemke, W. Dreybrodt, J. A. Shelnutt, J. M. E. Quirke, and R. Schweitzer-Stenner, "Polarized Raman dispersion spectroscopy probes planar and non-planar distortions of $\mathrm{Ni}(\mathrm{II})$ porphyrins with different peripheral substituents," Journal of Raman Spectroscopy, vol. 29, no. 10-11, pp. 945-953, 1998.

[67] W. Jentzen, X. Song, and J. A. Shelnutt, "Structural characterization of synthetic and protein-bound porphyrins in terms of the lowest-frequency normal coordinates of the macrocycle," Journal of Physical Chemistry B, vol. 101, no. 9, pp. 1684-1699, 1997.

[68] J. D. Hobbs and J. A. Shelnutt, "Conserved nonplanar heme distortions in cytochromes c," Journal of Protein Chemistry, vol. 14, no. 1, pp. 19-25, 1995.

[69] J. A. Shelnutt, X. Song, J. Ma, S. Jia, W. Jentzen, and C. J. Medforth, "Nonplanar porphyrins and their significance in proteins," Chemical Society Reviews, vol. 27, no. 1, pp. 31-41, 1998.

[70] J. A. Shelnutt, J. D. Hobbs, S. A. Majumder et al., "Resonance Raman spectroscopy of non-planar nickel porphyrins," Journal of Raman Spectroscopy, vol. 23, p. 523, 1992.

[71] R. Schweitzer-Stenner, C. Lemke, R. Haddad et al., "Conformational distortions of metalloporphyrins with electronwithdrawing NO2 substituents at different meso positions. A structural analysis by polarized resonance Raman dispersion spectroscopy and molecular mechanics calculations," Journal of Physical Chemistry A, vol. 105, no. 27, pp. 6680-6694, 2001.

[72] M. Alessi, A. M. Hagarman, J. B. Soffer, and R. SchweitzerStenner, "In-plane deformations of the heme group in native and nonnative oxidized cytochrome $c$ probed by resonance 
Raman dispersion spectroscopy," Journal of Raman Spectroscopy, vol. 42, no. 5, pp. 917-925, 2011.

[73] A. Hagarman, C. J. Wallace, M. M. Laberge, and R. SchweitzerStenner, "Out-of-plane deformations of the heme group in different ferrocytochrome c proteins probed by resonance Raman spectroscopy," Journal of Raman Spectroscopy, vol. 39, no. 12, pp. 1848-1858, 2008.

[74] R. Schweitzer-Stenner, D. Wedekind, and W. Dreybrodt, "Correspondence of the $\mathrm{pK}$ values of oxyHb-titration states detected by resonance Raman scattering to kinetic data of ligand dissociation and association," Biophysical Journal, vol. 49, no. 5, pp. 1077-1088, 1986.

[75] R. Schweitzer-Stenner, D. Wedekind, and W. Dreybrodt, "Detection of the heme perturbations caused by the quaternary $\mathrm{R} \rightarrow \mathrm{T}$ transition in oxyhemoglobin trout IV by resonance Raman scattering.," Biophysical Journal, vol. 55, no. 4, pp. 703712, 1989.

[76] S. A. Roberts, A. Weichsel, Y. Qiu, J. A. Shelnutt, F. A. Walker, and W. R. Montfort, "Ligand-induced heme ruffling and bent NO geometry in ultra-high-resolution structures of nitrophorin 4," Biochemistry, vol. 40, no. 38, pp. 11327-11337, 2001.

[77] M. K. Safo, F. A. Walker, A. M. Raitsimring et al., "Axial ligand orientation in iron(III) porphyrinates: effect of axial $\pi$-acceptors. Characterization of the low-spin complex $\left[\mathrm{Fe}(\mathrm{TPP})(4-\mathrm{CNPy})_{2}\right] \mathrm{ClO}_{4}$," Journal of the American Chemical Society, vol. 116, no. 17, pp. 7760-7770, 1994.

[78] L. V. Michel, T. Ye, S. E. J. Bowman et al., "Heme attachment motif mobility tunes cytochrome c redox potential," Biochemistry, vol. 46, no. 42, pp. 11753-11760, 2007.

[79] M. D. Liptak, X. Wen, and K. L. Bren, "NMR and DFT investigation of heme ruffling: functional implications for cytochrome c," Journal of the American Chemical Society, vol. 132, no. 28, pp. 9753-9763, 2010.

[80] J. M. Friedman, D. L. Rousseau, and F. Adar, "Excited state lifetimes in cytochromes measured from Raman scattering data: evidence for iron-porphyrin interactions," Proceedings of the National Academy of Sciences of the United States of America, vol. 74, no. 7, pp. 2607-2611, 1977.

[81] I. Dragomir, A. Hagarman, C. Wallace, and R. SchweitzerStenner, "Optical band splitting and electronic perturbations of the heme chromophore in cytochrome $\mathrm{c}$ at room temperature probed by visible electronic circular dichroism spectroscopy," Biophysical Journal, vol. 92, no. 3, pp. 989-998, 2007.

[82] R. Schweitzer-Stenner, J. P. Gorden, and A. Hagarman, “Asymmetric band profile of the Soret band of deoxymyoglobin is caused by electronic and vibronic perturbations of the heme group rather than by a doming deformation," Journal of Chemical Physics, vol. 127, no. 13, Article ID 135103, 2007.

[83] M.-C. Hsu and R. W. Woody, "The origin of the heme cotton effects in myoglobin and hemoglobin," Journal of the American Chemical Society, vol. 93, no. 14, pp. 3515-3525, 1971.

[84] G. Blauer, N. Sreerama, and R. W. Woody, "Optical activity of hemoproteins in the soret region. Circular dichroism of the heme undecapeptide of cytochrome c in aqueous solution," Biochemistry, vol. 32, no. 26, pp. 6674-6679, 1993.

[85] C. Kiefl, N. Sreerama, R. Haddad et al., "Heme distortions in sperm-whale carbonmonoxy myoglobin: correlations between rotational strengths and heme distortions in MD-generated structures," Journal of the American Chemical Society, vol. 124, no. 13, pp. 3385-3394, 2002.

[86] G. J. Pielak, K. Oikawa, A. Grant Mauk, M. Smith, and C. M. Kay, "Elimination of the negative soret cotton effect of cytochrome $\mathrm{c}$ by replacement of the invariant phenylalanine using site-directed mutagenesis," Journal of the American Chemical Society®, vol. 108, no. 10, pp. 2724-2727, 1986.

[87] R. Santucci and F. Ascoli, "The soret circular dichroism spectrum as a probe for the heme Fe(III)- Met(80) axial bond in horse cytochrome c," Journal of Inorganic Biochemistry, vol. 68, no. 3, pp. 211-214, 1997.

[88] A. Hagarman, L. Duitch, and R. Schweitzer-Stenner, "The conformational manifold of ferricytochrome $c$ explored by visible and far-UV electronic circular dichroism spectroscopy," Biochemistry, vol. 47, no. 36, pp. 9667-9677, 2008.

[89] C. J. Abel, R. A. Goldbeck, R. F. Latypov, H. Roder, and D. S. Kliger, "Conformational equilibration time of unfolded protein chains and the folding speed limit," Biochemistry, vol. 46, no. 13, pp. 4090-4099, 2007.

[90] E. S. Manas, J. M. Yanderkooi, and K. A. Sharp, "The effects of protein environment on the low temperature electronic spectroscopy of cytochrome c and microperoxidase-11," Journal of Physical Chemistry B, vol. 103, no. 30, pp. 6334-6348, 1999.

[91] R. Schweitzer-Stenner, M. Levantino, A. Cupane, C. Wallace, M. Laberge, and Q. Huang, "Functionally relevant electricfield induced perturbations of the prosthetic group of yeast ferrocytochrome $\mathrm{c}$ mutants obtained from a vibronic analysis of low-temperature absorption spectra," Journal of Physical Chemistry B, vol. 110, no. 24, pp. 12155-12161, 2006.

[92] U. Kubitscheck, W. Dreybrodt, and R. Schweitzer-Stenner, "Detection of heme-distortions in ferri- and ferrocyto-chrome C by resonance Raman scattering," Spectroscopy Letters, vol. 19, no. 6, pp. 681-690, 1986.

[93] G. R. Moore and R. J. Williams, "Nuclear-magnetic-resonance studies of ferrocytochrome c. pH and temperature dependence," European Journal of Biochemistry, vol. 103, no. 3, pp. 513-521, 1980.

[94] D. S. Cohen and G. J. Pielak, "Stability of yeast iso-1ferricytochrome c as a function of $\mathrm{pH}$ and temperature," Protein Science, vol. 3, no. 8, pp. 1253-1260, 1994.

[95] R. Varhač, M. Antalik, and M. Baro, "Effect of temperature and guanidine hydrochloride on ferrocytochrome c at neutral $\mathrm{pH}$," Journal of Biological Inorganic Chemistry, vol. 9, pp. 12-22, 2004.

[96] E. Droghetti, S. Oellerich, P. Hildebrandt, and G. Smulevich, "Heme coordination states of unfolded ferrous cytochrome c," Biophysical Journal, vol. 91, no. 8, pp. 3022-3031, 2006.

[97] A. M. Berghuis and G. D. Brayer, "Oxidation state-dependent conformational changes in cytochrome c," Journal of Molecular Biology, vol. 223, no. 4, pp. 959-976, 1992.

[98] G. G. Silkstone, C. E. Cooper, D. Svistunenko, and M. T. Wilson, "EPR and optical spectroscopic studies of Met80X mutants of yeast ferricytochrome c. models for intermediates in the alkaline transition," Journal of the American Chemical Society, vol. 127, no. 1, pp. 92-99, 2005.

[99] P. Baistrocchi, L. Banci, I. Bertini, P. Turano, K. L. Bren, and H. B. Gray, "Three-dimensional solution structure of Saccharomyces cerevisiae reduced Iso-1-cytochrome c," Biochemistry, vol. 35, no. 43, pp. 13788-13796, 1996.

[100] L. Banci, I. Bertini, J. G. Huber, G. A. Spyroulias, and P. Turano, "Solution structure of reduced horse heart cytochrome c," The Journal of Biological Inorganic Chemistry, vol. 4, no. 1, pp. 21-31, 1999.

[101] Y. W. Bai, T. R. Sosnick, L. C. Mayne, and S. W. Englander, "Protein folding intermediates: native-state hydrogen exchange," Science, vol. 269, no. 5221, pp. 192-197, 1995. 
[102] L. Banci, I. Bertini, H. B. Gray et al., "Solution structure of oxidized horse heart cytochrome c," Biochemistry, vol. 36, no. 32, pp. 9867-9877, 1997.

[103] D. Verbaro, A. Hagarman, J. Soffer, and R. Schweitzer-Stenner, "The $\mathrm{pH}$ dependence of the $695 \mathrm{~nm}$ charge transfer band reveals the population of an intermediate state of the alkaline transition of ferricytochrome c at low ion concentrations," Biochemistry, vol. 48, no. 13, pp. 2990-2996, 2009.

[104] C. B. Anfinsen, "Principles that govern the folding of protein chains," Science, vol. 181, no. 4096, pp. 223-230, 1973.

[105] H. Theorell and Å. Åkesson, "Studies on cytochrome c. III. Titration curves," Journal of the American Chemical Society, vol. 63, no. 7, pp. 1818-1820, 1941.

[106] H. J. Dyson and J. K. Beattie, "Spin state and unfolding equilibria of ferricytochrome c in acidic solutions," The Journal of Biological Chemistry, vol. 257, no. 5, pp. 2267-2273, 1982.

[107] Y. Goto, N. Takahashi, and A. L. Fink, "Mechanism of acidinduced folding of proteins," Biochemistry, vol. 29, no. 14, pp. 3480-3488, 1990.

[108] C. Indiani, G. De Sanctis, F. Neri, H. Santos, G. Smulevich, and M. Coletta, "Effect of $\mathrm{pH}$ on axial ligand coordination of cytochrome c" from Methylophilus methylotrophus and horse heart cytochrome c," Biochemistry, vol. 39, no. 28, pp. 8234$8242,2000$.

[109] S. Zhong, D. L. Rousseau, and S.-R. Yeh, "Modulation of the folding energy landscape of cytochrome $c$ with salt," Journal of the American Chemical Society, vol. 126, no. 43, pp.13934-13935, 2004.

[110] W. Colón and H. Roder, "Kinetic intermediates in the formation of the cytochrome c molten globule," Nature Structural Biology, vol. 3, no. 12, pp. 1019-1025, 1996.

[111] F. I. Rosell, J. C. Ferrer, and A. G. Mauk, "Proton-linked protein conformational switching: definition of the alkaline conformational transition of yeast iso-1-ferricytochrome c," Journal of the American Chemical Society, vol. 120, no. 44, pp. 11234-11245, 1998.

[112] S. Döpner, P. Hildebrandt, F. I. Resell, and A. G. Mauk, "Alkaline conformational transitions of ferricytochrome $\mathrm{c}$ studied by resonance Raman spectroscopy," Journal of the American Chemical Society, vol. 120, no. 44, pp. 11246-11255, 1998.

[113] P. D. Barker and A. G. Mauk, "pH-linked conformational regulation of a metalloprotein oxidation-reduction equilibrium: electrochemical analysis of the alkaline form of cytochrome $c$," Journal of the American Chemical Society, vol. 114, no. 10, pp. 3619-3624, 1992.

[114] M. M. Cherney and B. E. Bowler, "Protein dynamics and function: making new strides with an old warhorse, the alkaline conformational transition of cytochrome c," Coordination Chemistry Reviews, vol. 255, no. 7-8, pp. 664-677, 2011.

[115] P. Weinkam, J. Zimmermann, L. B. Sagle et al., "Characterization of alkaline transitions in ferricytochrome $\mathrm{C}$ using carbondeuterium infrared probes," Biochemistry, vol. 47, no. 51, pp. 13470-13480, 2008.

[116] L. A. Davis, A. Schejter, and G. P. Hess, "Alkaline isomerization of oxidized cytochrome c," The Journal of Biological Chemistry, vol. 249, no. 8, pp. 2624-2632, 1974.

[117] C. Blouin, J. G. Guillemette, and C. J. A. Wallace, "Resolving the individual components of a $\mathrm{pH}$-induced conformational change," Biophysical Journal, vol. 81, no. 4, pp. 2331-2338, 2001.

[118] G. Battistuzzi, M. Borsari, and M. Sola, "Medium and temperature effects on the redox chemistry of cytochrome c," European
Journal of Inorganic Chemistry, vol. 2001, no. 12, pp. 2989-3004, 2001.

[119] G. Battistuzzi, M. Borsari, L. Loschi, A. Martinelli, and M. Sola, "Thermodynamics of the alkaline transition of cytochrome $c$," Biochemistry, vol. 38, no. 25, pp. 7900-7907, 1999.

[120] L. Hoang, H. Maity, M. M. G. Krishna, Y. Lin, and S. W. Englander, "Folding units govern the cytochrome c alkaline transition," Journal of Molecular Biology, vol. 331, no. 1, pp. 3743, 2003.

[121] M. Assfalg, I. Bertini, A. Dolfi et al., "Structural model for an alkaline form of ferricytochrome c," Journal of the American Chemical Society, vol. 125, no. 10, pp. 2913-2922, 2003.

[122] J. B. Soffer, The Folded, Partially Folded, Misfolded, and Unfolded Conformations of Cytochrome C Probed by Optical Spectroscopy, Department of Chemistry, Drexel University, Philadelphia, Pa, USA, 2013

[123] Y. P. Myer, "Conformation of cytochromes. III. Effect of urea, temperature, extrinsic ligands, and $\mathrm{pH}$ variation on the conformation of horse heart ferricytochrome c," Biochemistry, vol. 7, no. 2, pp. 765-776, 1968.

[124] M. F. Jeng, S. W. Englander, G. A. Elöve, H. Roder, and A. J. Wand, "Structural description of acid-denatured cytochrome c by hydrogen exchange and 2D NMR," Biochemistry, vol. 29, no. 46, pp. 10433-10437, 1990.

[125] Y. Goto, Y. Hagihara, D. Hamada, M. Hoshimo, and I. Nishil, "Acid-induced unfolding and refolding transitions of cytochrome $c$ : a three-state mechanism in $\mathrm{H}_{2} \mathrm{O}$ and $\mathrm{D}_{2} \mathrm{O}$," Biochemistry, vol. 32, no. 44, pp. 11878-11885, 1993.

[126] G. Battistuzzi, C. A. Bortolotti, M. Bellei et al., "Role of Met80 and Tyr67 in the low-pH conformational equilibria of cytochrome c," Biochemistry, vol. 51, no. 30, pp. 5967-5978, 2012.

[127] J. B. Soffer and R. Schweitzer-Stenner, "Near-exact enthalpyentropy compensation governs the thermal unfolding of protonation states of oxidized cytochrome C," Journal of Biological Inorganic Chemistry, In press.

[128] Y. P. Myer and A. Pande, "Circular dichroism studies of hemoproteins and heme models," in The Porphyrins, D. Dolphin, Ed., pp. 271-322, Academic Press, New York, NY, USA, 1978.

[129] G. Taler, A. Schejter, G. Navon, I. Vig, and E. Margoliash, "The nature of the thermal equilibrium affecting the iron coordination of ferric cytochrome c," Biochemistry, vol. 34, no. 43, pp. 14209-14212, 1995.

[130] R. Schweitzer-Stenner, R. Shah, A. Hagarman, and I. Dragomir, "Conformational substates of horse heart cytochrome c exhibit different thermal unfolding of the heme cavity," Journal of Physical Chemistry B, vol. 111, no. 32, pp. 9603-9607, 2007.

[131] A. Spilotros, M. Levantino, and A. Cupane, "Conformational substates of ferricytochrome $\mathrm{c}$ revealed by combined optical absorption and electronic circular dichroism spectroscopy at cryogenic temperature," Biophysical Chemistry, vol. 147, no. 1-2, pp. 8-12, 2010.

[132] H. Frauenfelder, P. W. Fenimore, G. Chen, and B. H. McMahon, "Protein folding is slaved to solvent motions," Proceedings of the National Academy of Sciences of the United States of America, vol. 103, no. 42, pp. 15469-15472, 2006.

[133] R. Lumry and S. Rajender, "Enthalpy-entropy compensation phenomena in water solutions of proteins and small molecules: a ubiquitous property of water," Biopolymers-Peptide Science Section, vol. 9, no. 10, pp. 1125-1227, 1970.

[134] L. Liu, C. Yang, and Q. Guo, "A study on the enthalpy-entropy compensation in protein unfolding," Biophysical Chemistry, vol. 84 , no. 3, pp. 239-251, 2000. 
[135] L. Liu and Q. Guo, "Isokinetic relationship, isoequilibrium relationship, and enthalpy-entropy compensation," Chemical Reviews, vol. 101, no. 3, pp. 673-695, 2001.

[136] J. R. Beasley, D. F. Doyle, L. Chen, D. S. Cohen, B. R. Fine, and G. J. Pielak, "Searching for quantitative entropy-enthalpy compensation among protein variants," Proteins: Structure, Function and Genetics, vol. 49, no. 3, pp. 398-402, 2002.

[137] E. B. Starikov and B. Nordén, "Enthalpy-entropy compensation: a phantom or something useful?" Journal of Physical Chemistry B, vol. 111, no. 51, pp. 14431-14435, 2007.

[138] D. J. Segel, D. Eliezer, V. Uversky, A. L. Fink, K. O. Hodgson, and S. Doniach, "Transient dimer in the refolding kinetics of cytochrome c characterized by small-angle X-ray scattering," Biochemistry, vol. 38, no. 46, pp. 15352-15359, 1999.

[139] G. Balakrishnan, Y. Hu, and T. G. Spiro, "His26 protonation in cytochrome $\mathrm{c}$ triggers microsecond $\beta$-sheet formation and heme exposure: implications for apoptosis," Journal of the American Chemical Society, vol. 134, no. 46, pp. 19061-19069, 2012.

[140] P. P. Parui, M. S. Deshpande, S. Nagao et al., "Formation of oligomeric cytochrome $\mathrm{c}$ during folding by intermolecular hydrophobic interaction between $\mathrm{N}$ - and C-terminal $\alpha$-helices," Biochemistry, vol. 52, no. 48, pp. 8732-8744, 2013.

[141] C. M. Dobson, "Protein misfolding, evolution and disease," Trends in Biochemical Sciences, vol. 24, no. 9, pp. 329-332, 1999.

[142] A. Filosa and A. M. English, "Probing local thermal stabilities of bovine, horse, and tuna ferricytochromes $\mathrm{c}$ at $\mathrm{pH}$ 7," Journal of Biological Inorganic Chemistry, vol. 5, no. 4, pp. 448-454, 2000.

[143] E. Margoliash and J. Lustgarten, "Interconversion of horse heart cytochrome c monomer and polymers," The Journal of Biological Chemistry, vol. 237, pp. 3397-3405, 1962.

[144] S. Hirota, Y. Hattori, S. Nagao et al., "Cytochrome c polymerization by successive domain swapping at the C-terminal helix," Proceedings of the National Academy of Sciences of the United States of America, vol. 107, no. 29, pp. 12854-12859, 2010.

[145] F. Rousseau, J. W. H. Schymkowitz, and L. S. Itzhaki, "The unfolding story of three-dimensional domain swapping," Structure, vol. 11, no. 3, pp. 243-251, 2003.

[146] Z. Wang, T. Matsuo, S. Nagao, and S. Hirota, "Peroxidase activity enhancement of horse cytochrome c by dimerization," Organic and Biomolecular Chemistry, vol. 9, no. 13, pp. 47664769, 2011.

[147] J. B. Soffer, E. Fradkin, L. A. Pandiscia, and R. SchweitzerStenner, "The (not completely irreversible) population of a misfolded state of cytochrome c under folding conditions," Biochemistry, vol. 52, no. 8, pp. 1397-1408, 2013.

[148] W. A. Eaton and R. M. Hochstrasser, "Single-crystal spectra of ferrimyoglobin complexes in polarized light," The Journal of Chemical Physics, vol. 49, no. 3, pp. 985-995, 1968.

[149] W. A. Eaton and R. M. Hochstrasser, "Electronic spectrum of single crystals of ferricytochrome-c," The Journal of Chemical Physics, vol. 46, no. 7, pp. 2533-2539, 1967.

[150] S. Döpner, P. Hildebrandt, F. I. Rosell et al., "The structural and functional role of lysine residues in the binding domain of cytochrome c in the electron transfer to cytochrome c oxidase," European Journal of Biochemistry, vol. 261, no. 2, pp. 379-391, 1999.

[151] B. D. Howes, C. B. Schiødt, K. G. Welinder et al., "The quantum mixed-spin heme state of barley peroxidase: a paradigm for class III peroxidases," Biophysical Journal, vol. 77, no. 1, pp. 478492, 1999.
[152] B. D. Howes, A. C. Feis, M. Indiani, M. Morzocchi, and G. Smulevich, "Formation of two types of low spin heme in horseradosh peroxidase isoenzyme A2 at low temperatures," Journal of Biological Inorganic Chemistry, vol. 276, pp. 4070440711, 2000.

[153] A. Feis, B. D. Howes, C. Indiani, and G. Smulevich, "Resonance Raman and electronic absorption spectra of horseradish peroxidase isozyme A2: evidence for a quantum-mixed spin species," Journal of Raman Spectroscopy, vol. 29, no. 10-11, pp. 933-938, 1998.

[154] M. M. Maltempo, "Visible absorption spectra of quantum mixed spin ferric heme proteins," Biochimica et Biophysica Acta, vol. 434, no. 2, pp. 513-518, 1976.

[155] Q. Huang, K. Szigeti, J. Fidy, and R. Schweitzer-Stenner, "Structural disorder of native horseradish peroxidase $\mathrm{C}$ probed by resonance Raman and low-temperature optical absorption spectroscopy," Journal of Physical Chemistry B, vol. 107, no. 12, pp. 2822-2830, 2003.

[156] P. Weinkam, F. E. Romesberg, and P. G. Wolynes, "Chemical frustration in the protein folding landscape: grand canonical ensemble simulations of cytochrome c," Biochemistry, vol. 48, no. 11, pp. 2394-2402, 2009.

[157] W. Zheng, N. P. Schafer, and P. G. Wolynes, "Frustration in the energy landscapes of multidomain protein misfolding," Proceedings of the National Academy of Sciences of the United States of America, vol. 110, no. 5, pp. 1680-1685, 2013.

[158] J. D. Cortese, A. L. Voglino, and C. R. Hackenbrock, "Multiple conformations of physiological membrane-bound cytochrome c," Biochemistry, vol. 37, no. 18, pp. 6402-6409, 1998.

[159] S. Berezhna, H. Wohlrab, and P. M. Champion, "Resonance Raman investigations of cytochrome c conformational change upon interaction with the membranes of intact and $\mathrm{CA}^{2+}$. exposed mitochondria," Biochemistry, vol. 42, no. 20, pp. 6149$6158,2003$.

[160] D. H. Murgida, P. Hildebrandt, J. Wei, Y. F. He, H. Liu, and D. H. Waldeck, "Surface-enhanced resonance raman spectroscopic and electrochemical study of cytochrome c bound on electrodes through coordination with pyridinyl-terminated selfassembled monolayers," The Journal of Physical Chemistry B, vol. 108, no. 7, pp. 2261-2269, 2004.

[161] D. H. Murgida and P. Hildebrandt, "Electron-transfer processes of cytochrome c at interfaces. New insights by surface-enhanced resonance Raman spectroscopy," Accounts of Chemical Research, vol. 37, no. 11, pp. 854-861, 2004.

[162] H. Wackerbarth and P. Hildebrandt, "Redox and conformational equilibria and dynamics of Cytochrome $c$ at high electric fields," ChemPhysChem, vol. 4, no. 7, pp. 714-724, 2003.

[163] S. Monari, D. Millo, A. Ranieri et al., "The impact of ureainduced unfolding on the redox process of immobilised cytochrome c," Journal of Biological Inorganic Chemistry, vol. 15, no. 8, pp. 1233-1242, 2010.

[164] A. Ranieri, G. Battistuzzi, M. Borsari et al., "A bis-histidineligated unfolded cytochrome $\mathrm{c}$ immobilized on anionic SAM shows pseudo-peroxidase activity," Electrochemistry Communications, vol. 14, no. 1, pp. 29-31, 2012.

[165] M. Rytömaa and P. K. J. Kinnunen, "Evidence for two distinct acidic phospholipid-binding sites in cytochrome c," The Journal of Biological Chemistry, vol. 269, no. 3, pp. 1770-1774, 1994.

[166] E. Kalanxhi and C. J. A. Wallace, "Cytochrome c impaled: investigation of the extended lipid anchorage of a soluble protein to mitochondrial membrane models," Biochemical Journal, vol. 407, no. 2, pp. 179-187, 2007. 
[167] M. Rytömaa, P. Mustonen, and P. K. J. Kinnunen, "Reversible, nonionic, and $\mathrm{pH}$-dependent association of cytochrome $\mathrm{c}$ with cardiolipin-phosphatidylcholine liposomes," Journal of Biological Chemistry, vol. 267, no. 31, pp. 22243-22248, 1992.

[168] C. L. Bergstrom, P. A. Beales, Y. Lv, T. K. Vanderlick, and J. T. Groves, "Cytochrome $c$ causes pore formation in cardiolipincontaining membranes," Proceedings of the National Academy of Sciences of the United States of America, vol. 110, no. 16, pp. 6269-6274, 2013.

[169] C. Kawai, F. M. Prado, G. L. C. Nunes, P. Di Mascio, A. M. Carmona-Ribeiro, and I. L. Nantes, "pH-dependent interaction of cytochrome $\mathrm{c}$ with mitochondrial mimetic membranes: the role of an array of positively charged amino acids," The Journal of Biological Chemistry, vol. 280, no. 41, pp. 34709-34717, 2005.

[170] E. J. Snider, J. Muenzner, J. R. Toffey, Y. J. Hong, and E. V. Pletneva, "Multifaceted effects of ATP on cardiolipin-bound cytochrome c," Biochemistry, vol. 52, no. 6, pp. 993-995, 2013.

[171] E. V. Pletneva, H. B. Gray, and J. R. Winkler, "Nature of the cytochrome c molten globule," Journal of the American Chemical Society, vol. 127, no. 44, pp. 15370-15371, 2005.

[172] Y. Hong, J. Muenzner, S. K. Grimm, and E. V. Pletneva, “Origin of the conformational heterogeneity of cardiolipin-bound cytochrome c," Journal of the American Chemical Society, vol. 134, no. 45, pp. 18713-18723, 2012.

[173] J. Hanske, J. R. Toffey, A. M. Morenz, A. J. Bonilla, K. H. Schiavoni, and E. V. Pletneva, "Conformational properties of cardiolipin-bound cytochrome c," Proceedings of the National Academy of Sciences of the United States of America, vol. 109, no. 1, pp. 125-130, 2012.

[174] F. Sinibaldi, L. Fiorucci, A. Patriarca et al., "Insights into cytochrome c-cardiolipin interaction. Role played by ionic strength," Biochemistry, vol. 47, no. 26, pp. 6928-6935, 2008.

[175] L. A. Pandiscia and R. Schweitzer-Stenner, "Salt as a catalyst in the mitochondria: returning cytochrome $c$ to its Native State after it misfolds on the surface of cardiolipin containing membranes," Chemical Communications, vol. 50, no. 28, pp. 3674-3676, 2014. 

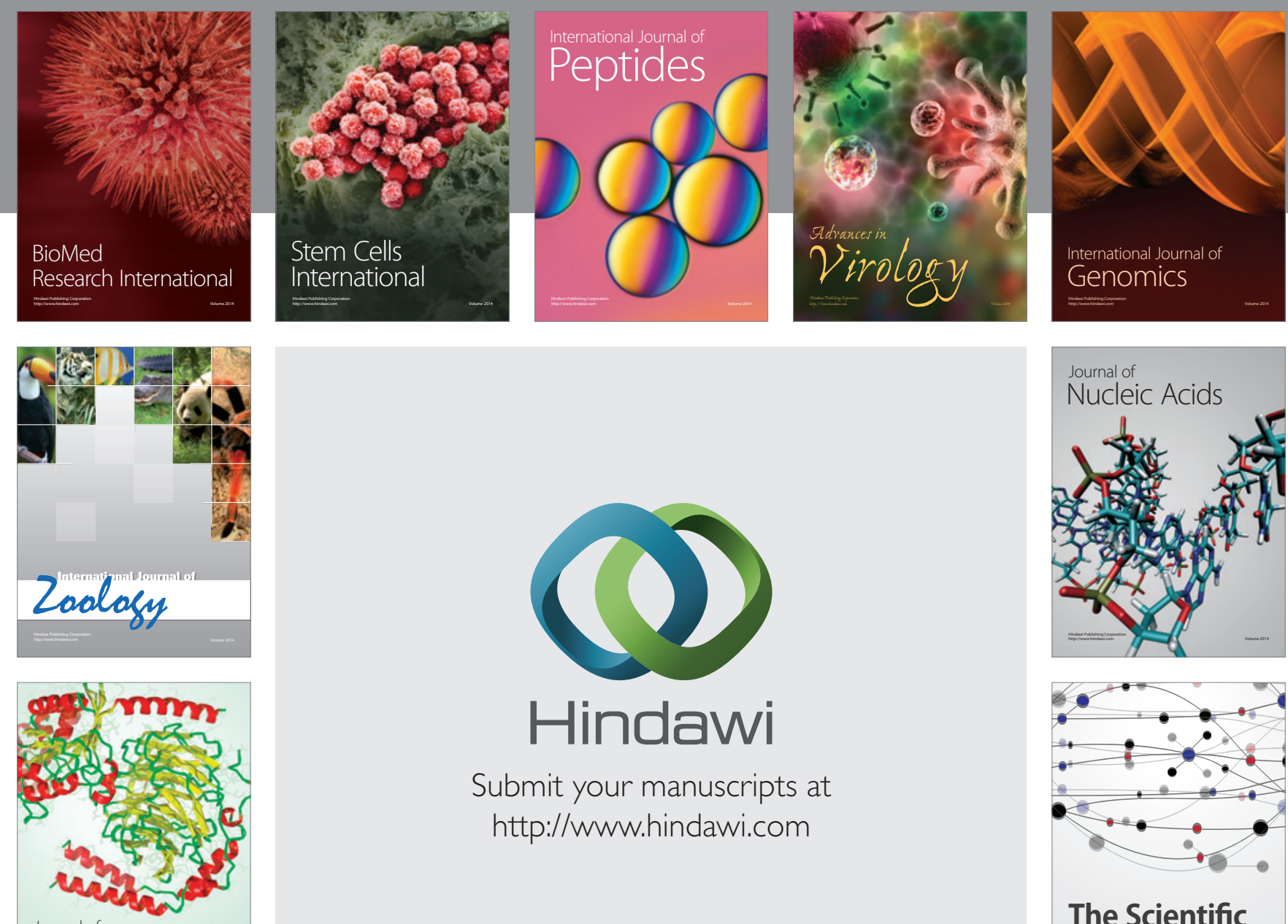

Submit your manuscripts at

http://www.hindawi.com

Journal of
Signal Transduction
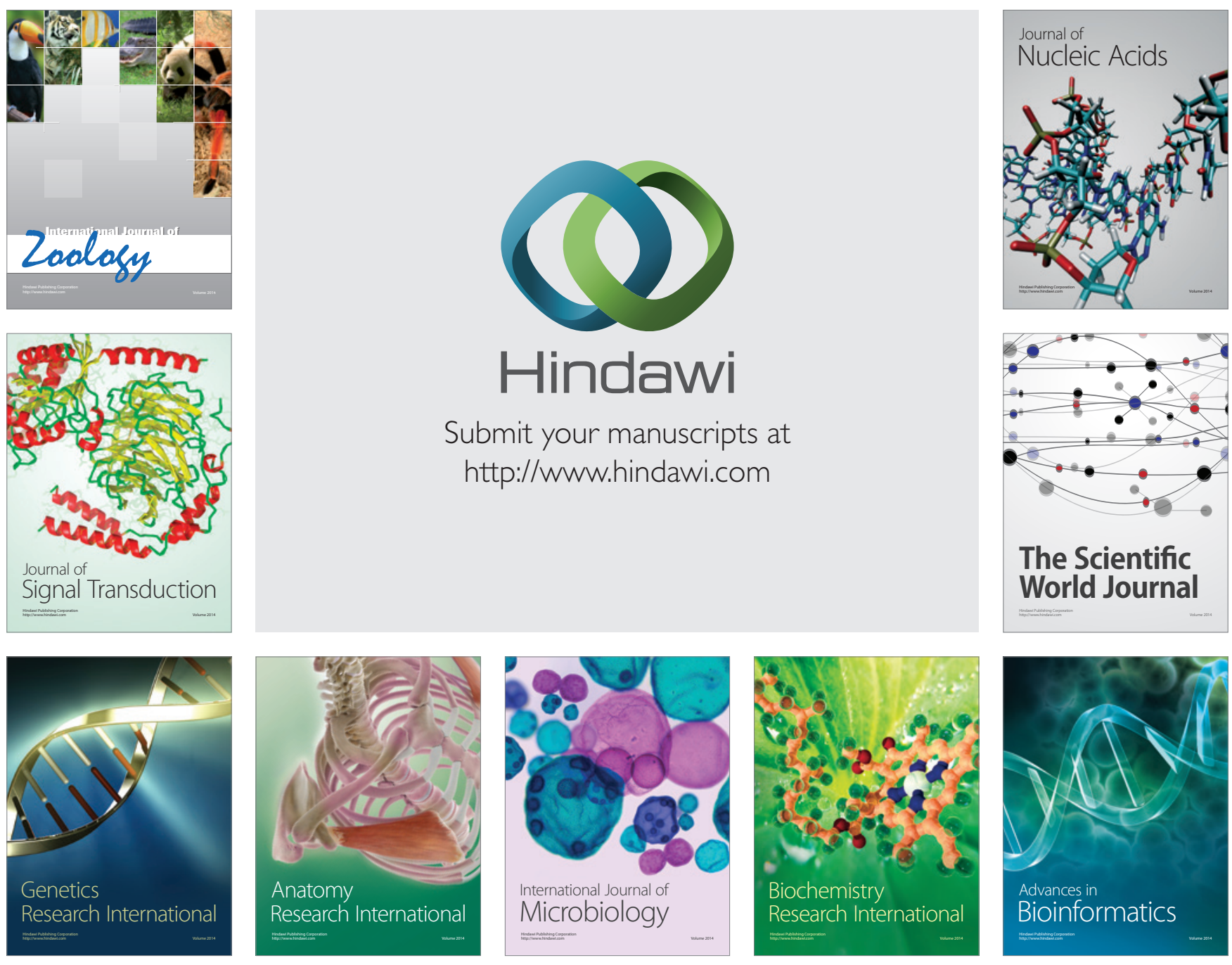

The Scientific World Journal
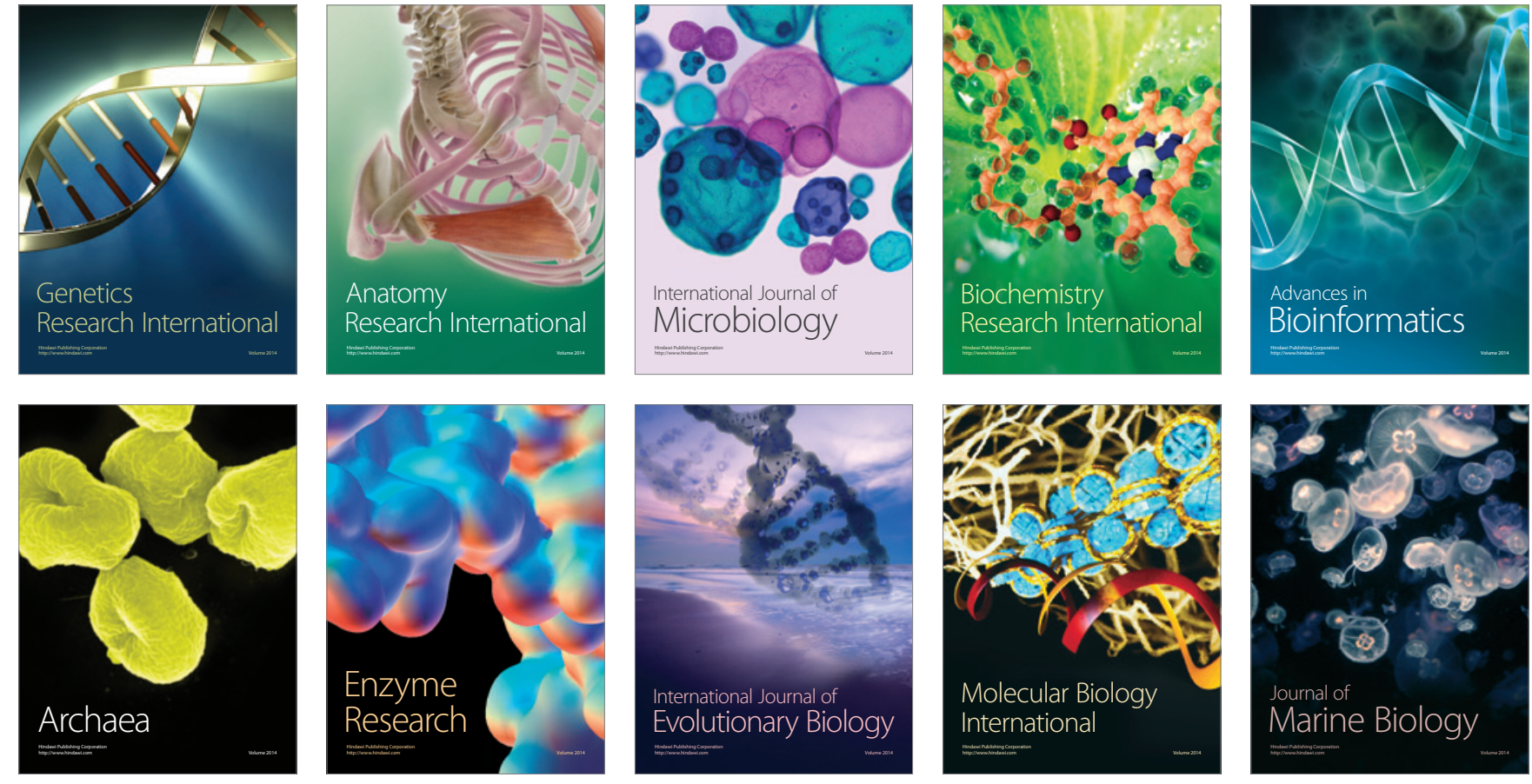